\title{
Developmental and psychoneuroimmunological mechanisms in depression
}

Citation for published version (APA):

Pujol-Lopez, Y. (2015). Developmental and psychoneuroimmunological mechanisms in depression.

[Doctoral Thesis, Maastricht University]. Maastricht University. https://doi.org/10.26481/dis.20151030yp

Document status and date:

Published: 01/01/2015

DOI:

10.26481/dis.20151030yp

Document Version:

Publisher's PDF, also known as Version of record

\section{Please check the document version of this publication:}

- A submitted manuscript is the version of the article upon submission and before peer-review. There can be important differences between the submitted version and the official published version of record.

People interested in the research are advised to contact the author for the final version of the publication, or visit the DOI to the publisher's website.

- The final author version and the galley proof are versions of the publication after peer review.

- The final published version features the final layout of the paper including the volume, issue and page numbers.

Link to publication

\footnotetext{
General rights rights.

- You may freely distribute the URL identifying the publication in the public portal. please follow below link for the End User Agreement:

www.umlib.nl/taverne-license

Take down policy

If you believe that this document breaches copyright please contact us at:

repository@maastrichtuniversity.nl

providing details and we will investigate your claim.
}

Copyright and moral rights for the publications made accessible in the public portal are retained by the authors and/or other copyright owners and it is a condition of accessing publications that users recognise and abide by the legal requirements associated with these

- Users may download and print one copy of any publication from the public portal for the purpose of private study or research.

- You may not further distribute the material or use it for any profit-making activity or commercial gain

If the publication is distributed under the terms of Article $25 \mathrm{fa}$ of the Dutch Copyright Act, indicated by the "Taverne" license above, 
MAASTRICHT UNIVERSITY

\section{Developmental and psychoneuroimmunological mechanisms in depression}

Yara Pujol López 
Copyright (C) Yara Pujol López, Maastricht, 2015

Developmental and psychoneuroimmunological mechanisms in depression

All rights reserved. No part of this book may be reproduced or transmitted in any form or by any means, without permission in writing, from the copyright holder.

ISBN: 978-94-6203-939-1

Cover design: Jo Stevens

Printed by CPI Koninklijke Wöhrmann 


\section{Developmental and psychoneuroimmunological mechanisms in depression}

\section{DISSERTATION}

To obtain the degree of Doctor at

Maastricht University

on the authority of the Rector Magnificus, Prof. Dr. L.L.G. Soete in accordance with the decision of the Board of Deans,

to be defended in public

on Friday $30^{\text {th }}$ of October 2015 , at 10.00 hours

by

\section{Yara Pujol López}




\section{Supervisor:}

Prof. Dr. H.W.M. Steinbusch

\section{Co-supervisors:}

Dr. D.L.A. van den Hove

Dr. G. Kenis

Dr. AM. Myint (Ludwig Maximilian University of Munich, Germany)

\section{Assessment committee:}

Prof. Dr. M. de Baets (Chairman)

Prof. Dr. B. Kramer

Prof. Dr. K-P. Lesch (University of Würzburg, Germany)

Prof. Dr. H. Drexhage (Erasmus University Rotterdam, The Netherlands)

Prof. Dr. N. Hellings (Hasselt University, Belgium)

The studies described in this dissertation were partially supported by the Commission of European Communities 7th Framework Programme collaborative project MOODINFLAME (grant No. 22963) and by FP 7 MarieCurie IAAP Project Psych-Aid (Grant No. 286334). Financial support for the publication of this dissertation by the "School for Mental Health and Neuroscience, Maastricht University" is gratefully acknowledged. 
Pels meus avis Joan i Josefina, para mis yayos Carmen y Luis y para mi ahijada Irina. 



\section{Contents}

Chapter 1

9

General Introduction

Chapter 2

Prenatal maternal infection and offspring brain development:

programming of adult psychopathology

\section{Chapter 3}

Effects of subcutaneous lipopolysaccharide (LPS) injection on

gestational length and intra-uterine and neonatal mortality in mice

Chapter 4

87

Quinolinic acid (QUIN)-immunoreactivity in the naïve mouse brain

\section{Chapter 5}

111

Effects of prenatal polyinosinic:polycytidylic acid (Poly I:C) exposure on global histone deacetylase (HDAC) and DNA methyltransferase (DNMT) activity in the mouse brain

\section{Chapter 6}

Depressive symptoms during interferon-alpha (IFN- $\alpha$ ) therapy in humans associated with changes in the kynurenine pathway

\section{Chapter 7}

General Discussion

Summary

Valorization

Acknowledgements

Curriculum Vitae

Publications 



\section{Chapter 1}

General introduction

Yara Pujol López 


\section{Background}

The expression of psychiatric disorders includes a wide range of different categories of afflictions that are currently classified by two widely established systems: in the International Classification of Diseases (ICD-11) formulated by the World Health Organization (WHO) and in the Diagnostic and Statistical Manual of Mental Disorders (DSM-V) formulated by the American Psychiatric Association (APA) (Cuthbert and Insel 2013). Among psychiatric disorders, major depression is the leading cause of disability (WHO 2012) comprising several symptoms, such as sadness, anxiety, hopelessness, loss of interest, fatigue, and insomnia (Health 2010). To recognise the large impact of depression in our society, it is reported that currently more than 350 million people are estimated to suffer from depression including an increasing number of young persons (WHO 2012). Apart from the increasing number of people suffering from depression and the devastating effect on the quality of life of patients, the disease is associated with a high social, economic and societal burden. Depression is associated with divorce and suicide, among others (Wolfersdorf 2008). In addition, people suffering from depression reduce their productivity in the workplace, which has a considerable economic impact (Gilbody, Bower et al. 2012, McTernan, Dollard et al. 2013).

\section{Diagnosis and treatment of major depression}

Nowadays, the diagnosis of major depression is still based on a clinical psychiatric interview, complemented with specific questionnaires based on 
ICD-10 or DSM-V criteria. Suitable biomarkers for depression are still missing. The subjective nature of diagnosing depression is further complicated by patient-specific behaviour: the patient may or may not collaborate and often describes his/her symptoms in a personal manner and the doctor needs to be able to understand and interpret them. Moreover, a diagnosis is often difficult to make because of the many different ways in which clinical depression can be manifested.

Regarding treatment, it is essentially based on psychotherapy and the use of antidepressants. Since the 1950s, different generations of antidepressants were launched on the market with the purpose of increasing several neurotransmitter levels, involving serotonin $(5-\mathrm{HT})$ and noradrenaline (NA) among others. However, to date, no significant improvement in the rates of relapse has been observed after using different therapeutic options. Some patients do not even respond to any form of treatment. Others do, but often show recurrence. Altogether, there is a high need to develop more effective treatment options, which requires more insight in the molecular mechanisms underlying this debilitating disorder.

\section{Hypotheses on the pathogenesis of depression}

As from the beginning of the last century, several hypotheses have been suggested to explain the pathogenesis of depression. Although they are addressed in detail in Chapter 2, an overview of the hypotheses is provided below.

First, the monoamine hypothesis was proposed, associating depression to reduced levels of monoamines, such as 5-HT and NA (Schildkraut 1995). This 
theory was supported by the fact that the serendipitously discovered antidepressants such as tricyclic antidepressants (TCAs) and monoamine oxidase inhibitors (MAOIs) increase synaptic monoamine levels, and dominated the field of depression research for several decades. However, this hypothesis could not explain why medication that increases monoamine levels is not effective for all depressive patients, and it neither clarified the pathophysiology of depression (Hirschfeld 2000, Hindmarch 2002). In addition, changes in monoamines have not been consistently described and there is a delay between the moment when antidepressants are administered and after which clinical effects are observed (Stahl 2000). Altogether, it suggests that other mechanisms mediating the monoamine system may be involved, such as changes in receptors or in epigenetic mechanisms. As such, alternative theories were formulated. The hypothalamic-pituitary-adrenal (HPA) axis or corticosteroid hypothesis of depression suggests that hyperactivity of the HPA axis and concomitant high levels of corticosteroids may be involved in the development of depression. Hyperactivity of the HPA axis is indeed often observed in depressed patients (Swaab, Bao et al. 2005) and antidepressants are known to induce changes in the levels of glucocorticoid receptors (GR), which sustains this hypothesis (Anacker, Zunszain et al. 2011). Moreover, evidence shows increased production and secretion of glucocorticoids in depressed patients (Gillespie and Nemeroff 2005) and impairment of the GR, e.g. glucocorticoid resistance and concomitant alteration of the feedback regulation of the HPA axis (Pariante and Miller 2001). Another theory links depression with a deficit in neurotrophic factor signaling. Specifically, studies focusing on 
brain-derived neurotrophic factor (BDNF) showed hippocampal atrophy and reduced serum BDNF levels in depressed patients. Interestingly, a decrease in BDNF levels was reversed after antidepressant treatment (Shimizu, Hashimoto et al. 2003, Gonul, Akdeniz et al. 2005, Yoshimura, Mitoma et al. 2007). In addition, antidepressants have been reported to normalize BDNF levels in the hippocampus in postmortem brain tissue of patients suffering from major depression (Chen, Dowlatshahi et al. 2001).

The immune system has also been related to depression, initially formulated in the macrophage theory of depression. According to this hypothesis, excessive secretion of cytokines by macrophages and monocytes would cause depression (Smith 1991). Several studies corroborate this theory showing increased inflammatory markers in some depressive patients (Maes 1999, Howren, Lamkin et al. 2009). For instance, evidence shows increased interleukin (IL)-6, C-reactive protein and tumor necrosis factor alpha. (TNFa) (Dinan 2009). In addition, as a comorbid illness, major depression is prevalent in cancer (Snyderman and Wynn 2009) and cardiac patients (Huffman, Celano et al. 2013), disorders often associated with inflammation. Moreover, administration of interferon-alpha (IFN- $\alpha$ ), a pro-inflammatory cytokine commonly used to treat hepatitis $C$, is reported to induce major depression in up to $45 \%$ of hepatitis C patients (Asnis and De La Garza 2006). Interestingly, anti-inflammatory drugs are able to alleviate depression in some patients (Muller 2013), and commonly used antidepressants show anti-inflammatory properties, such as a reduction in pro-inflammatory cytokines (Kim, Suh et al. 2002, Cavanagh and Mathias 2008). Following the link between inflammation and depression, the cytokine hypothesis of 
depression was formulated. This hypothesis suggests cytokines as the key mechanism responsible for inducing depressive behaviour via an increased degradation of tryptophan (TRP) to neurotoxic catabolites along the kynurenine pathway (Maes 2008). First TRP can be metabolised to 5-HT by tryptophan hydroxylase (TPH) or to kynurenine by tryptophan 2,3dioxygenase (TDO) or indoleamine 2,3-dioxygenase (IDO) (Leonard and Myint 2009). TDO is mainly located in the liver, whereas IDO has been detected in many tissues, such as brain, placenta, pancreas, among others (Moffett and Namboodiri 2003). Kynurenine is further metabolised by kynurenine aminotransferases (KATs) to kynurenic acid (KYNA), which acts as a N-methyl-D-aspartate glutamate (NMDA) glutamate receptor antagonist in the brain. Alternatively, kynurenine can be metabolised to 3hydroxykynurenine (3HK) by kynurenine-3-monooxygenase (KMO), after which it is further degraded to 3-hydroxyanthranilic acid (3HAA) by kynureninase (KYNase). 3HAA is involved in the synthesis of adenosine triphosphate (ATP), mainly in the liver, and degraded into quinolinic acid (QUIN) by 3-hydroxyanthranilic acid oxygenase (3HAO). QUIN is a NMDA glutamate receptor agonist and can be degraded into nicotinamide adenine dinucleotide (NAD) (Sperner-Unterweger, Miller et al. 2002, Myint 2012) (see Figure 1). Interestingly, various enzymes involved in the kynurenine pathway can be modulated by cytokines. For instance, both interferon gamma (IFN- $\gamma$ ) and tumor necrosis factor alpha (TNF- $\alpha$ ) activate IDO and $\mathrm{KMO}$, whereas TDO activity can be further enhanced by glucocorticoids (Myint 2012). Thus, activation of the kynurenine pathway will lead to depletion of TRP, and consequently of 5-HT. In addition, KYNA is known as a 
potential neuroprotective metabolite, whereas QUIN and 3HK induce neurotoxic effects, such as excitotoxic neuronal cell loss and convulsions (Nemeth, Toldi et al. 2005). Along this line, the neurodegeneration hypothesis of depression was formulated which integrates the above mentioned theories, the kynurenine pathway and its connection with cytokines, the kynurenine metabolites, 5-HT as well as NMDA-glutamatergic neurotransmission (Myint and Kim 2003). Specifically, this hypothesis emphasizes the importance of a balance between kynurenic and quinolinic acid, considered as a neuroprotective and neurotoxic metabolites respectively. 


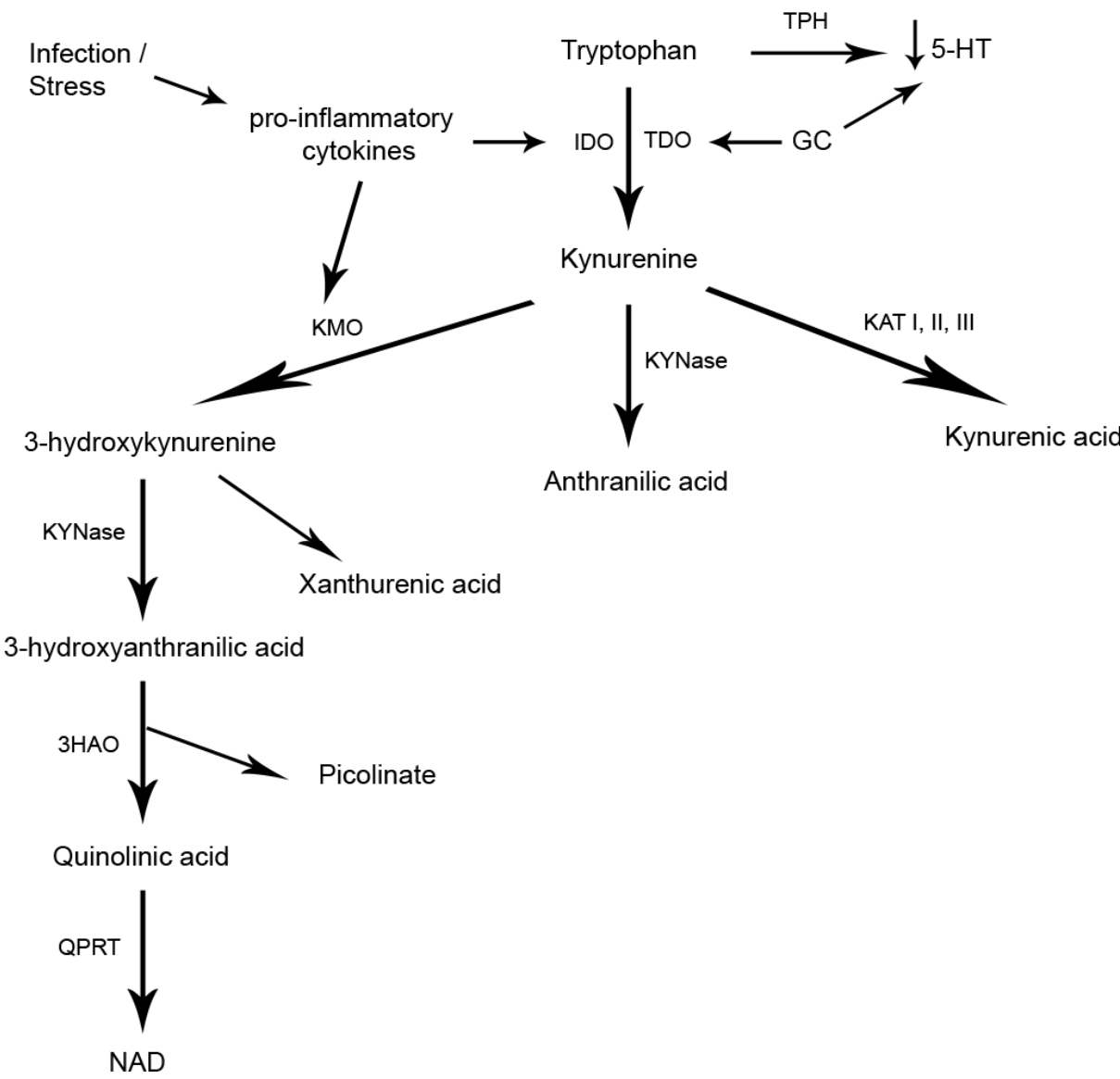

Figure 1. Schema of tryptophan metabolism. See text for details. Abbreviations: TPH, tryptophan hydroxylase; 5-HT, serotonin; GC, glucocorticoids; IDO, indoleamine 2,3-dioxygenase; TDO, tryptophan 2,3-dioxygenase; KAT, kynurenine aminotransferase; KMO, kynurenine-3monooxygenase; KYNase, kynureninase; 3HAO, 3-hydroxyanthranilic acid oxygenase; QPRT, quinolinate phosphoribosyl transferase; NAD, nicotinamide adenine dinucleotide. 


\section{Prenatal maternal infection and depression in the offspring}

Several insults during pregnancy have been related to psychopathology in the offspring (Weinstock 2001, Zammit, Thomas et al. 2009). Interestingly, inflammatory processes have been suggested as key mediators underlying the relationship between prenatal environmental insults and the development of psychiatric disorders later in life (Patterson 2002, Meyer, Yee et al. 2007). As such, several animal studies have been performed in order to investigate the link prenatal maternal infection and offspring psychopathology (Beumer, Gibney et al. 2012), although they particularly focused on schizophrenia (Zuckerman, Rehavi et al. 2003, Ozawa, Hashimoto et al. 2006, Meyer and Feldon 2009). The exact mechanisms that mediate the relation between prenatal maternal infection and depression in the offspring remain to be elucidated. Epigenetic processes likely candidate mechanisms in this respect.

\section{Epigenetic mechanisms}

The term epigenetics describes heritable changes in gene expression that do not involve alterations of the DNA sequence. The most-studied epigenetic mechanisms to date involve DNA methylation and histone modifications, which are addressed below.

DNA methylation implies the methylation of cytosine in DNA forming 5methylcytosine $(5 \mathrm{mC})$. The enzymes responsible for this process are DNA methyltransferases (DNMTs). DNMT1 mediates the conservation of methylation during DNA replication, whereas DNMT3 regulate de novo 
methylation. DNA methylation is involved in the regulation of gene expression by methylation of $\mathrm{CpG}$ dinucleotides and by methyl $\mathrm{CpG}$-binding domain (MBD)-containing protein family members. Generally, DNA methylation results in the suppression of gene expression (Das and Chai 2013).

Histones are proteins that together with the DNA and other proteins form the chromatin, whose basic unit is the nucleosome. A nucleosome consists of 2 of each classical histone proteins (H2A, H2B, H3 and H4) wrapping 147 base pairs of DNA. Post-translational modifications in the tail of these histones, such as acetylation, deacetylation, methylation, phosphorylation, ubiquitylation and others, affect chromatin structure. The concomitant changes in compaction of the chromatin modulate interactions between DNA sequences and transcription factors, DNA binding proteins, ncRNAs, thus altering gene expression (Das and Chai 2013). The most common modifications are histone acetylation and deacetylation, regulated by histone acetyltransferases (HAT) and histone deacetyltransferases (HDAC), respectively. Acetylation of histones results in heterochromatin, which facilitates the access and binding of transcription factors and additional nuclear proteins, promoting transcription. On the contrary, deacetylation of histones leads to compacted euchromatin and transcriptional repression. Interestingly, infections can modulate epigenetic processes in many ways (Bierne, Hamon et al. 2012), whereas epigenetic regulation is known to target genes involved in cell progression, survival, inflammation and immunity. Thus, being a key player in the development and differentiation of the nervous system that may explain the response of the central nervous 
system (CNS) at a molecular level, in case of infection and concomitant injury.

\section{Aim and outline of this thesis}

The overarching aim of this thesis is to investigate the intricate role of inflammation in depression. More specifically, we hypothesized that alterations in the kynurenine pathway are causally involved in mediating changes in inflammation-induced depression-related phenotypes. Moreover, we postulated epigenetic changes as mechanisms underlying the association between prenatal inflammation and adult psychopathology.

In Chapter 2 of this thesis we review evidence on how exposure to prenatal maternal infection may interfere with offspring brain development. Moreover, sex-differences in the response to prenatal maternal infection as well as treatment options are discussed.

Chapter 3 highlights how prenatal infection using an endotoxin contained in bacterial cell walls, lipopolysaccharide (LPS), affects gestational length and intra-uterine and neonatal mortality in mice.

In Chapter 4, we provide an analysis of QUIN in the naïve mouse brain in relation to affect-related behavior.

Chapter $\mathbf{5}$ examines alterations in epigenetic mechanisms, such as global HDAC and DNMT activity in the mouse brain after prenatal exposure to polyinosinic:polycytidylic acid (Poly I:C). 
In Chapter 6, we explore alterations in diverse enzymes and metabolites of the kynurenine pathway in relation to depression scores of IFN- $\alpha$ treated patients.

Finally, Chapter 7 provides a general conclusion for our findings.

Of note, for our mouse studies, we used the C57BL/6JRccHsd mouse strain (Harlan Laboratories, Germany). This is a well characterized mouse strain frequently used in behavioral research. C57BI/6J mice show low 'emotionality', high open-field exploration, high locomotor activity and only short time of immobility during the forced swim test (Thompson 1953, Nikulina, Skrinskaya et al. 1991). This specific strain was also chosen because they still carry the gene that encodes for the enzyme nicotinamide nucleotide transhydrogenase, which is missing in the original C57BL/6 strain. This enzyme is important for our field of research because it is involved in the last reaction of the kynurenine pathway, where it converts NAD+ to NADP+ which in turn is used for biosynthesis and detoxification. In addition, this strain does not lack $\alpha$-synuclein, which belongs to a family of structural proteins which is highly expressed in the brain. For these reasons, the C57BL/6JRccHsd strain is more wild-type like, which is important to ensure that brain morphology and functioning is not affected by any of these genetic mutations. 


\section{References}

Anacker, C., P. A. Zunszain, L. A. Carvalho and C. M. Pariante (2011). "The glucocorticoid receptor: Pivot of depression and of antidepressant treatment?" Psychoneuroendocrinology 36(3): 415-425.

Asnis, G. M. and R. De La Garza, 2nd (2006). "Interferon-induced depression in chronic hepatitis C: a review of its prevalence, risk factors, biology, and treatment approaches." J Clin Gastroenterol 40(4): 322-335.

Beumer, W., S. M. Gibney, R. C. Drexhage, L. Pont-Lezica, J. Doorduin, H. C. Klein, J. Steiner, T. J. Connor, A. Harkin, M. A. Versnel and H. A. Drexhage (2012). "The immune theory of psychiatric diseases: a key role for activated microglia and circulating monocytes." Journal of Leukocyte Biology 92(5): 959-975.

Bierne, H., M. Hamon and P. Cossart (2012). "Epigenetics and bacterial infections." Cold Spring Harb Perspect Med 2(12): a010272.

Cavanagh, J. and C. Mathias (2008). "Inflammation and its relevance to psychiatry." Advances in Psychiatric Treatment 14(4): 248-255.

Chen, B., D. Dowlatshahi, G. M. MacQueen, J. F. Wang and L. T. Young (2001). "Increased hippocampal BDNF immunoreactivity in subjects treated with antidepressant medication." Biol Psychiatry 50(4): 260-265.

Cuthbert, B. and T. Insel (2013). "Toward the future of psychiatric diagnosis: the seven pillars of RDoC." BMC Medicine 11(1): 126.

Das, N. D. and Y. G. Chai (2013). Neuroinflammation on the Epigenetics of Neural Stem Cells, INTECH Open Access Publisher.

Dinan, T. G. (2009). "Inflammatory markers in depression." Curr Opin Psychiatry 22(1): 32-36.

Gilbody, S., P. Bower and J. Rick (2012). "Better care for depression in the workplace: integrating occupational and mental health services." The British Journal of Psychiatry 200(6): 442-443.

Gillespie, C. F. and C. B. Nemeroff (2005). "Hypercortisolemia and depression." Psychosom Med 67 Suppl 1: S26-28.

Gonul, A. S., F. Akdeniz, F. Taneli, O. Donat, C. Eker and S. Vahip (2005). "Effect of treatment on serum brain-derived neurotrophic factor levels in depressed patients." Eur Arch Psychiatry Clin Neurosci 255(6): 381-386.

Health, N. C. C. f. M. (2010). Depression: the treatment and management of depression in adults (updated edition), British Psychological Society.

Hindmarch, I. (2002). "Beyond the monoamine hypothesis: mechanisms, molecules and methods." European Psychiatry 17: 294-299.

Hirschfeld, R. M. (2000). "History and evolution of the monoamine hypothesis of depression." J Clin Psychiatry 61 Suppl 6: 4-6. 
Howren, M. B., D. M. Lamkin and J. Suls (2009). "Associations of depression with Creactive protein, IL-1, and IL-6: a meta-analysis." Psychosom Med 71(2): 171-186.

Huffman, J. C., C. M. Celano, S. R. Beach, S. R. Motiwala and J. L. Januzzi (2013). "Depression and Cardiac Disease: Epidemiology, Mechanisms, and Diagnosis." Cardiovascular Psychiatry and Neurology 2013: 14.

Kim, Y. K., I. B. Suh, H. Kim, C. S. Han, C. S. Lim, S. H. Choi and J. Licinio (2002). "The plasma levels of interleukin-12 in schizophrenia, major depression, and bipolar mania: effects of psychotropic drugs." Mol Psychiatry 7(10): 11071114.

Leonard, B. E. and A. Myint (2009). "The psychoneuroimmunology of depression." Hum Psychopharmacol 24(3): 165-175.

Maes, M. (1999). "Major depression and activation of the inflammatory response system." Adv Exp Med Biol 461: 25-46.

Maes, M. (2008). "The cytokine hypothesis of depression: inflammation, oxidative \& nitrosative stress (IO\&NS) and leaky gut as new targets for adjunctive treatments in depression." Neuro Endocrinol Lett 29(3): 287-291.

McTernan, W. P., M. F. Dollard and A. D. LaMontagne (2013). "Depression in the workplace: An economic cost analysis of depression-related productivity loss attributable to job strain and bullying." Work \& Stress 27(4): 321-338.

Meyer, U. and J. Feldon (2009). "Prenatal exposure to infection: a primary mechanism for abnormal dopaminergic development in schizophrenia." Psychopharmacology (Berl) 206(4): 587-602.

Meyer, U., B. K. Yee and J. Feldon (2007). "The neurodevelopmental impact of prenatal infections at different times of pregnancy: the earlier the worse?" Neuroscientist 13(3): 241-256.

Moffett, J. R. and M. A. A. Namboodiri (2003). "Tryptophan and the immune response." Immunol Cell Biol 81(4): 247-265.

Muller, N. (2013). "The role of anti-inflammatory treatment in psychiatric disorders." Psychiatr Danub 25(3): 292-298.

Myint, A. M. (2012). "Kynurenines: from the perspective of major psychiatric disorders." FEBS Journal 279(8): 1375-1385.

Myint, A. M. and Y. K. Kim (2003). "Cytokine-serotonin interaction through IDO: a neurodegeneration hypothesis of depression." Med Hypotheses 61(5-6): 519-525.

Nemeth, H., J. Toldi and L. Vecsei (2005). "Role of kynurenines in the central and peripherial nervous systems." Current neurovascular research 2(3): 249260. 
Nikulina, E., J. Skrinskaya and N. Popova (1991). "Role of genotype and dopamine receptors in behaviour of inbred mice in a forced swimming test." Psychopharmacology 105(4): 525-529.

Ozawa, K., K. Hashimoto, T. Kishimoto, E. Shimizu, H. Ishikura and M. Iyo (2006). "Immune activation during pregnancy in mice leads to dopaminergic hyperfunction and cognitive impairment in the offspring: a neurodevelopmental animal model of schizophrenia." Biol Psychiatry 59(6): 546-554.

Pariante, C. M. and A. H. Miller (2001). "Glucocorticoid receptors in major depression: relevance to pathophysiology and treatment." Biological Psychiatry 49(5): 391-404.

Patterson, P. H. (2002). "Maternal infection: window on neuroimmune interactions in fetal brain development and mental illness." Curr Opin Neurobiol 12(1): 115-118.

Schildkraut, J. J. (1995). "The catecholamine hypothesis of affective disorders: a review of supporting evidence. 1965." J Neuropsychiatry Clin Neurosci 7(4): 524-533; discussion 523-524.

Shimizu, E., K. Hashimoto, N. Okamura, K. Koike, N. Komatsu, C. Kumakiri, M. Nakazato, H. Watanabe, N. Shinoda and S.-i. Okada (2003). "Alterations of serum levels of brain-derived neurotrophic factor (BDNF) in depressed patients with or without antidepressants." Biological psychiatry 54(1): 7075.

Smith, R. S. (1991). "The macrophage theory of depression." Med Hypotheses 35(4): 298-306.

Snyderman, D. and D. Wynn (2009). "Depression in Cancer Patients." Primary Care: Clinics in Office Practice 36(4): 703-719.

Sperner-Unterweger, B., C. Miller, B. Holzner, A. Laich, B. Widner, W. W. Fleischhacker and D. Fuchs (2002). "Immunologic alterations in schizophrenia: neopterin, L-kynurenine, tryptophan and T-cell subsets in the acute stage of illness." Pteridines 13(1): 9-14.

Stahl, S. M. (2000). Essential Psychopharmacology: Neuroscientific Basis and Practical Applications, Cambridge University Press.

Swaab, D. F., A. M. Bao and P. J. Lucassen (2005). "The stress system in the human brain in depression and neurodegeneration." Ageing Res Rev 4(2): 141-194.

Thompson, W. R. (1953). "The inheritance of behaviour: behavioural differences in fifteen mouse strains." Can J Psychol 7(4): 145-155.

Weinstock, M. (2001). "Alterations induced by gestational stress in brain morphology and behaviour of the offspring." Prog Neurobiol 65(5): 427451. 
WHO (2012). "Depression: A Global Crisis. World Mental Health Day, October 10 2012. World Federation for Mental Health. Available: http://www.who.int/mental health/management/depression/wfmh pap er depression wmhd 2012.pdf."

Wolfersdorf, M. (2008). "[Depression and suicide]." Bundesgesundheitsblatt Gesundheitsforschung Gesundheitsschutz 51(4): 443-450.

Yoshimura, R., M. Mitoma, A. Sugita, H. Hori, T. Okamoto, W. Umene, N. Ueda and J. Nakamura (2007). "Effects of paroxetine or milnacipran on serum brainderived neurotrophic factor in depressed patients." Prog Neuropsychopharmacol Biol Psychiatry 31(5): 1034-1037.

Zammit, S., K. Thomas, A. Thompson, J. Horwood, P. Menezes, D. Gunnell, C. Hollis, D. Wolke, G. Lewis and G. Harrison (2009). Maternal tobacco, cannabis and alcohol use during pregnancy and risk of adolescent psychotic symptoms in offspring.

Zuckerman, L., M. Rehavi, R. Nachman and I. Weiner (2003). "Immune activation during pregnancy in rats leads to a postpubertal emergence of disrupted latent inhibition, dopaminergic hyperfunction, and altered limbic morphology in the offspring: a novel neurodevelopmental model of schizophrenia." Neuropsychopharmacology 28(10): 1778-1789. 


\section{Chapter 2}

\section{Prenatal maternal infection and offspring brain development: programming of adult psychopathology}

Yara Pujol Lopez; Daniel LA van den Hove; Harry WM Steinbusch; Gunter Kenis; Aye M Myint 


\section{Abstract}

This review addresses the possible effects of inflammation during pregnancy and its impact on offspring brain development. Activation of the immune system in pregnant women by e.g. infection or stress can result in different degrees of inflammatory responses, which in the long run may lead to the development of psychiatric disorders in the offspring. More specifically, during normal pregnancy, increased gonadal hormone levels induce immunosuppressive effects, through which women protect their foetus. This state of immune tolerance can be disrupted by the trigger of an inflammation, thereby impacting upon foetal brain development, concomitant with an increased predisposition to mental disorders in adulthood. As part of this process, maternal infection-induced cytokine production affects the action of enzymes, neurotransmitters, and hormones, a disturbance of which may have a lasting impact upon offspring brain function and behaviour. In addition, the kynurenine metabolism, in which the essential amino acid tryptophan is catabolised through several steps to quinolinic and kynurenic acid, has been shown to be affected in response to inflammation. Both metabolites are reported to regulate the balance between neurodegeneration and neuroprotection in the brain. Furthermore, a disturbance of the kynurenine metabolism also affects the availability of tryptophan, reducing the synthesis of serotonin. Moreover, part of the programming effects induced by inflammation during pregnancy is hypothesized to be mediated by epigenetic mechanism. As such, the present review will discuss the link between prenatal maternal infection, epigenetic processes, neuro-immune interactions and neurodegenerative 26 
damage to the central nervous system in view of the development of psychiatric disorders in the offspring. 


\section{Introduction}

Over the last decades, advances in psychiatric research have led to the notion that inflammatory processes and neuro-immune interactions are involved in the pathogenesis of mental disorders such as depression (see review by Gibney and Drexhage 2013). For example, evidence has shown that some individuals develop depression after exposure to infectious agents (see review by Dantzer, O'Connor et al. 2008, Benros, Waltoft et al. 2013), such as the Hepatitis C virus (see review by Zdilar, Franco-Bronson et al. 2000, Carta, Hardoy et al. 2007). Along similar lines, exposure to different pathogens during pregnancy, by impacting upon brain development and behaviour, has been shown to increase the risk for the development of psychiatric disorders in the offspring (see review by Boksa 2010). For instance, an elevated risk for the development of bipolar disorder, schizophrenia and autism has been observed in offspring of women who were infected with bacteria or viruses during gestation (Brown 2006, Atladottir, Thorsen et al. 2010, Parboosing, Bao et al. 2013).

To further investigate which mechanisms mediate the relation between infection during pregnancy and an increased risk to develop psychiatric disorders in the offspring, several studies have been performed using a variety of animal models, particularly in rats and mice. Examples include prenatal maternal exposure of rodents to lipopolysaccharide (LPS), polyinosinic:polycytidylic acid (Poly I:C) or the influenza virus. Both in response to maternal LPS and Poly I:C exposure rat offspring showed a deficit in prepulse inhibition (PPI) (Borrell, Vela et al. 2002, Wolff and Bilkey 28 
2008), which assesses the ability to gate sensory information, a reduction of which is observed in schizophrenia. In addition, after exposing pregnant mice to Poly I:C, memory deficits were observed in the offspring when subjected to the Morris water maze and novel object recognition test (Ozawa, Hashimoto et al. 2006, Meyer, Nyffeler et al. 2008). The exact mechanisms underlying brain dysfunction in offspring exposed to prenatal maternal infection remain to be elucidated though. This article provides a literature review about the link between prenatal maternal inflammation and the development of psychiatric disorders in the offspring and the possible mediators involved, including cytokines, neurotransmitters, neurotrophins and hormones as well as related epigenetic processes.

\subsection{Psychoneuroimmunology of depression.}

In the 90s of the last century, numerous studies reported immunological abnormalities observed in depressed subjects. Some studies found an increase of plasma pro-inflammatory cytokines levels (Interleukin [IL]-1, IL-6 and IL-8) as well as C-reactive protein (CRP) in depressed patients (Song, Lin et al. 1998, see review by Shelton and Miller 2011), whereas another study reported decreased levels of IL-1b and IL-2 when comparing healthy controls and depressive patients (Weizman, Laor et al. 1994). Several studies reported a reduction in NK cell toxicity, relative leucocytosis, slightly increased $\mathrm{CD} 4 / 8$ ratios, i. e. activation of cellular immunity, and reduced IL-6 levels in the cerebrospinal fluid (CSF) of patients with major depression (Stubner, Schon et al. 1999, see review 
by Zorrilla, Luborsky et al. 2001). On the other hand, other studies showed an increase in the number of cells with activation markers, in positive acute phase proteins, i.e. produced by the liver, induced by IL-6, and in the synthesis of prostaglandins and circulating soluble IL-2 receptors (see review by Maes 1999, Sluzewska 1999). Moreover, a decrease in negative acute phase proteins (Maes, Scharpe et al. 1992) and an overproduction of cytokines, such as the pro-inflammatory cytokines IL-1, IL-6 and IFN, have been reported (Maes, Smith et al. 1995). These levels of acute phase proteins, i.e. an increase in positive and a decrease in negative acute phase proteins are considered to be indicative of an inflammatory state (see review by Dunn, Swiergiel et al. 2005). Moreover, depression is known to be induced after administration of pro-inflammatory cytokines, such as IFN- $\alpha$ when treating an infectious disease or cancer (Raison, Capuron et al. 2006). Antidepressants have also been shown to possess anti-inflammatory and neuroprotective properties, by reducing pro-inflammatory cytokines (see review by Shelton and Miller 2011). Given the amount of evidence showing inflammation in some depressed patients, an inflammatory hypothesis of depression was first proposed by Smith (1991). This hypothesis is known as the "macrophage theory of depression" which related depression to immune activation (Smith 1991). To combat infection, fever and physiological changes in the body occur, concomitant with adaptive behavioural alterations. Cytokine activation, particularly of the pro-inflammatory type, regulates various hormones inducing behavioural alterations associated with the induction of sleep, 
anhedonia, irritability, etc., which, as a whole, is defined as "sickness behaviour" (Raison, Capuron et al. 2006). Depression and sickness behaviour have several symptoms in common including anhedonia, decreased appetite and activity, and disturbed sleep (see review by Hashmi, Aftab et al. 2013). This notion led to the "cytokine hypothesis of depression", which suggests a causal role for cytokines in inducing depressive behaviours by an increased metabolism of tryptophan to neurotoxic catabolites along the kynurenine pathway (Maes 2008).

\section{Pregnancy and the immune system}

\subsection{The immune system during normal pregnancy}

Classically, pregnancy has been regarded as a state of immune suppression in order to protect the foetus. However, over the last years, increasing evidence has shown that during gestation the immune system, though tightly regulated, is in fact very active (see review by Mor and Cardenas 2010). Generally, three different inflammatory phases are observed during pregnancy. First, during embryo implantation, placentation and the early stages of pregnancy, a pro-inflammatory environment dominates, whereas during mid-pregnancy an antiinflammatory milieu is observed, returning to a pro-inflammatory one at the third trimester and the end of pregnancy (see review by Amirchaghmaghi, Taghavi et al. 2013). A strong inflammatory response is required during the first phase of pregnancy to break the epithelium of the uterus stimulating the process of implantation and subsequent 
steps including the invasion and replacement of endometrial tissue (see review by Dekel, Gnainsky et al. 2010). Second, a shift to an antiinflammatory allows a rapid foetal growth and development (see review by Mor and Cardenas 2010). Finally, a pro-inflammatory state is restored in order to stimulate the contraction of the uterus, delivery of the baby and rejection of the placenta (Mor 2008).

Along pregnancy, increased numbers of peripheral white blood cells have been detected in the peripheral maternal immune system (Veenstra van Nieuwenhoven, Bouman et al. 2002). In human decidua, elevated numbers of immune cells, e.g. natural killer (NK) cells, macrophages and regulatory $\mathrm{T}$ cells (Treg) are present during normal pregnancy (Bulmer, Pace et al. 1988, see review by Mor and Cardenas 2010). Specifically, NK cells were found to be increased during the first trimester of pregnancy (Hidaka, Amino et al. 1991). However, macrophages were elevated in early and in late gestation as well (Bulmer, Pace et al. 1988). Regarding Treg cells, an increase has been observed during both the first the second trimester of pregnancy (Somerset, Zheng et al. 2004).

2.2 Infection during pregnancy and inflammatory changes during gestation

As already mentioned, the exposure to an infectious agent during pregnancy is a major risk factor for neurodevelopmental brain damage (Golan, Lev et al. 2005). Maternal infection induces the production of cytokines and glucocorticoids (GCs), which have been hypothesized to 
be critically involved in mediating the development of e.g. neurodevelopmental disorders in the offspring (see review by Zhou 2012). Cytokines, such as IL-1B, IL-6 and tumor necrosis factor- $\alpha$ (TNF- $\alpha$ ) are induced in maternal and/or foetal tissues, with maternal cytokines being able to cross the placenta and to affect the development of the brain via different mechanisms (Ashdown, Dumont et al. 2006) for instance, through their direct involvement in brain development, such as lineage commitment, differentiation and survival of neurons and glial cells (see review by Zhou 2012). Furthermore, at a systemic level, cytokines represent key regulators of the inflammatory response triggering the immune response, provoking fever and initiating an elevated synthesis of acute phase proteins, whereas locally, they help to recruit inflammatory cells to inflammation sites (see review by Marques, Cizza et al. 2007). Moreover, cytokines are implicated in the modulation of a variety of processes in chemical synapses, such as neurotransmitter synthesis, storage, release, (re)uptake, degradation and interacting with specific neurotransmitter receptors as well as related signal transduction cascades, which are crucial in the regulation of CNS function (see review by Camacho-Arroyo, Lopez-Griego et al. 2009). Finally, they have also been shown to be involved in placental dysfunction and apoptosis of the trophoblast (see review by Boksa 2010).

Next to cytokines, GCs are known to represent key players mediating the effects of prenatal maternal infection. GCs which are considered immunosupressors that play a vital role in the control of the immune response (see review by Zhou 2012), are generally produced after 
activation of the hypothalamo-pituitary adrenal (HPA) axis, particularly in response to stress. In addition, maternal infection is known to result in elevated level of maternal blood GCs, concomitant with elevated foetal GC levels (see review by Zhou 2012). The concomitant effects of GCs are commented in detail below and in the section reviewing the stress and its involvement in depression.

To sum up, various factors impact upon the CNS in offspring exposed to prenatal maternal infection, including cytokines, stress hormones and neurotransmitters.

\subsubsection{Infection during pregnancy and the neuroimmune system axis}

\subsubsection{The corticosteroid hypothesis of depression}

This hypothesis postulates that chronic and/or excessive stress exposure and concomitant stress hormone dysregulation represent a major cause for developing depression. In view of inflammation, interestingly, there is evidence of increased HPA axis activity after infection (see review by Kronfol and Remick 2000) and of modulation of the axis by cytokines (Raison, Capuron et al. 2006). GCs control the secretion of corticotropin-releasing factor (CRF) and adrenocorticotrophic hormone (ACTH) executing a negative feedback at the level of the hypothalamus and pituitary, by activating the glucocorticoid receptor (GR) (see review by Leonard and Myint 2009). GCs like cortisol generally act as immunosuppressors via e.g. inhibition of IL-1, IL-2 and IL-6 production or secretion, depletion of 
CD4+ T cells, or promoting cell death (Maes, Smith et al. 1995). However, their effects on the immune system vary temporarily. Under physiological conditions they might enhance proinflammatory cytokines, whereas during the acute phase of the immune response, they are potent inhibitors of the synthesis and actions of central and peripheral cytokines (see review by Howerton and Bale 2012). Moreover, GCs produce different effects depending on the tissue type. For example, elevated GCs suppress the peripheral immune system, whereas they promote a proinflammatory state in immune cells in the brain (Howerton and Bale 2012). Some cytokines, such as TNF- $\alpha$, IL-1 and IL- 6 can directly activate the HPA axis inducing the hypothalamic release of CRF, and consequently ACTH and GCs (Rook, Hernandez-Pando et al. 1994). Cytokines can also exert direct effects on the pituitary and adrenal glands. However, peripheral cytokines can affect the HPA axis by other, more indirect ways too. For example, peripheral injection of IL-1 stimulates the hypothalamic release of noradrenaline that in turn stimulates the release of CRF (Dunn 1988). In addition, Th1 and pro-inflammatory cytokines are reported to decrease GR affinity, which contributes to the vicious circle of immune activation. These actions, together with impaired HPA axis control of cytokine secretion, are decisive in mediating disease progression (see review by Irwin 2002). In relation to pregnancy, GCs are able to pass the placenta (Edwards, Benediktsson et al. 1993) and exposure to normal physiological levels of GCs is in fact required for e.g. foetal 
development (Kitraki, Kittas et al. 1997), regulating brain processes such as subcellular reorganization of neuron-neuron and neuron-glia interactions (see review by Matthews 2000). Importantly, various mechanisms exist to prevent excessive exposure to GCs. For example, at the placenta, the enzyme 11ßhydroxysteroid dehydrogenase 2 (11ßHSD2) can convert GCs to inactive metabolites (Seckl 1997), thus protecting the developing foetus in case of elevated exposure to GCs, which could led to alterations in the neurodevelopment of the foetus (see review by Zhou 2012). Decreased placental 11ßHSD2 activity or expression has been associated with elevated GCs exposure in the foetus and birth complications in human and animals model (see review by Howerton and Bale 2012). In view of prenatal infection, rats prenatally exposed to LPS showed increased stress response, concomitant with elevated levels of anxiety-like behaviour. Moreover, after exposure to stress, offspring of LPS-injected dams, showed elevated corticosterone responses and reduced GR levels in the dorsal hippocampus (Lin, Lin et al. 2012). In offspring of rats injected with Poly I:C during pregnancy, stress-induced corticosterone levels were reduced compared to controls (Maayan, Ram et al. 2012). Other studies investigated corticosterone levels in rodents in both mothers and offspring after prenatal exposure to LPS. Whereas, one group reported increased levels of corticosterone in both pregnant dams and male offspring exposed to LPS (Enayati, Solati et al. 2012), other studies only found elevated maternal corticosterone levels and no 
changes in the offspring in response to prenatal maternal LPS exposure (Asiaei, Solati et al. 2011, Kirsten, Lippi et al. 2013). These differences may potentially be explained by the different timing of LPS injections during pregnancy and the different age of the offspring when studied.

\subsubsection{Neurotrophic hypothesis of depression}

The formulation of this hypothesis is based on the observation of reduced levels of neurotrophins like brain-derived neurotrophic factor (BDNF) in response to stress and in stress-related disorders like major depression, as well as the beneficial effects of antidepressants in this respect, e.g. by upregulating BDNF. Neurotrophic factors mediate neuronal survival, a wide range of neuronal and glial cell activities, e.g. axonal, dendritic growth, synaptic structure and plasticity, neurotransmitter expression, long-term potentiation, and various immune cell functions, such as immune cell activation, differentiation, migration, and local antigen presentation (Hohlfeld, Kerschensteiner et al. 2007). An additional function is the inhibition of neuronal death or degeneration. In fact, its levels and receptors are vigorously regulated in pathological conditions, which suggest the importance of neurotrophic factors in the response of neurons to degenerative or traumatic processes. They also regulate various important elements of inflammation, such as the migration of monocytes across the blood-brain barrier (BBB) and the release of pro-inflammatory cytokines (see review by Kerschensteiner, 
Stadelmann et al. 2003). The most-studied neurotrophic factor is BDNF, which is known to play an important role in regulation of neuronal survival, development and function (see review by Walker, Kim et al. 2014). Increased evidence reported the involvement of BDNF the pathogenesis of major depression as well as in mediating the action of antidepressants. For instance, reduced expression of its receptor (TrkB) has been observed in postmortem brain samples of depressed patients (Tripp, Oh et al. 2012). In addition, reduced levels of mature BDNF, but not of its precursor were shown in sera from patients suffering from depression (Karege, Perret et al. 2002, Yoshida, Ishikawa et al. 2012). After using antidepressants, elevated serum BDNF levels have been reported (Yoshimura, Mitoma et al. 2007). In view of infection during pregnancy, discordant results on BDNF have been reported. Offspring of pregnant rodents injected with LPS showed elevated brain BDNF levels (Gilmore, Jarskog et al. 2003, Golan, Lev et al. 2005), which may be explained as a consequence of a regenerative response to LPS. However, in another study, rat offspring prenatally exposed to LPS showed reduced BDNF in the hippocampus on postnatal day 21 and 90 (Lin and Wang 2014). When rats were exposed to Escherichia coli during gestation, increased expression of hippocampal BDNF and TrkB were observed in the offspring at postnatal 3 and 7 days (Jiang, Zhu et al. 2013). 


\subsubsection{Monoamine hypothesis of depression}

During the 1950s and 1960s of the last century, the monoamine hypothesis led research on psychiatric disorders. This hypothesis is centred on the impaired levels and activity of monoamines, particularly serotonin (5-hydroxytryptamine or 5-HT), in the development of depression (see review by Schildkraut 1965, Coppen 1967). Indeed, reduced blood 5-HT levels (Takahashi 1976) and reduced brain 5-HT transporter (5-HTT) availability (Willeit, PraschakRieder et al. 2000) have been found in patients suffering from depression. Besides $5-\mathrm{HT}$, reductions in brain dopamine levels (Martinot, Bragulat et al. 2001) and downregulation of the norepinephrine transporter (NET) have been shown in depressed patients (Klimek, Stockmeier et al. 1997). However, administration of antidepressants that increase monoamine levels, are not always effective (see review by Shelton 2006), indicating that this concept is oversimplified.

A closely related hypothesis, initially formulated in 1969, suggested the depletion of tryptophan, essential amino acid and precursor to 5$\mathrm{HT}$ due to an increased kynurenine synthesis as the cause for major depression. 5-HT is synthesized from the essential amino acid tryptophan, which is actively transported into the brain through the BBB (Fernstrom 1984). As such, the synthesis of 5-HT depends on the plasma levels of tryptophan (Song, Lin et al. 1998). However, this essential amino acid can be metabolised by two different pathways: to $5-\mathrm{HT}$ by the enzyme tryptophan hydroxylase (TPH) and to 
kynurenine by tryptophan 2,3-dioxygenase (TDO), mainly in the liver (Watanabe, Fujiwara et al. 1980), and by extrahepatic indoleamine 2,3-dioxygenase (IDO), e.g. in the brain (Moffett, Blinder et al. 1998). Whereas TDO is specific for tryptophan, IDO is also able to metabolize other substrates such as 5-HT and melatonin (Hayaishi 1976, Dang, Dale et al. 2000). Furthermore, TDO regulates whole basal serum tryptophan levels, IDO is up-regulated as a result of inflammatory conditions (Niinisalo, Raitala et al. 2008). In addition, TDO is stimulated by cortisol, whereas IDO is induced by proinflammatory cytokines, such as IFN- $\gamma$, IL- 6 and TNF $\alpha$ (see review by Maes, Leonard et al. 2011), and suppressed by anti-inflammatory cytokines (see review by Leonard and Myint 2009), such as IL-4 and IL-10 (Weiss, Murr et al. 1999).

Both TPH and IDO activity are directly and indirectly correlated with immune activation (Sperner-Unterweger, Miller et al. 2002). Whereas in physiological conditions tryptophan is metabolised mainly by TDO, an immune challenge suppresses TDO and stimulates IDO. IDO controls the quantity of tryptophan that will be metabolised to kynurenine (Maes, Meltzer et al. 1993) and increased levels of IDO have been associated with increased anxiety and impaired mood (see review by Maes 2011). In particular, two important metabolites, i.e. quinolinic acid (QUIN) and kynurenic acid (KYNA) are synthesised through the kynurenine pathway. Kynurenine is further metabolised to KYNA by kynurenine aminotransferases (KATs). Alternatively, kynurenine is metabolised to 3-hydroxykynurenine (3HK) by 
kynurenine-3-monooxygenase (KMO), after which it is further degraded to 3-hydroxyanthranilic acid (3HAA) by kynureninase. 3HAA is involved in the synthesis of adenosine triphosphate (ATP), mainly in the liver, and degraded into QUIN by 3-hydroxyanthranilic acid oxygenase (3HAO). QUIN is then degraded into nicotinamide adenine dinucleotide (NAD) (Sperner-Unterweger, Miller et al. 2002, see review by Myint 2012) (see Figure 1 in the introduction chapter). Both QUIN and KYNA directly mediate glutamate neurotransmission (Bay-Richter, Janelidze et al. 2011). These metabolites represent intercellular messengers with neuroactive, proapoptotic and immunoregulatory properties (see review by Zelante, Fallarino et al. 2009). Whereas KYNA is mainly known for its neuroprotective properties, QUIN is known to have more neurotoxic effects (see review by Nemeth, Toldi et al. 2005). Kynurenine is able to cross the BBB a very limited degree. In the brain, $40 \%$ of kynurenine is produced locally, whereas $60 \%$ originates from the periphery (Gál and Sherman 1980). QUIN induces neurotoxic effects through its action as agonist of $\mathrm{N}$-methyl-D-aspartate glutamate (NMDA) receptors, including excitotoxic neuronal cell loss and convulsions (see review by Nemeth, Toldi et al. 2005). Stimulation of extrasynaptic NMDA receptors reduces the production of BDNF, resulting in increased depressive-like behaviour (Raison and Miller 2013). It is proposed that the production and accumulation of QUIN in the CNS occurs in a variety of inflammatory neurological disorders as well as infections (Dang, Dale et al. 2000). For instance, higher QUIN levels 
were observed in activated microglia in the anterior cingulate cortex of suicide victims who suffered from depression (Raison and Miller 2013). QUIN also intensifies neurotoxic effects of pro-inflammatory cytokines (see review by Maes 2008). Other neurotoxic effects caused by QUIN are the induction of hippocampal cell death, selective necrosis of granule cells, destruction of postsynaptic elements and a reduction in cerebral cholinergic circuits (el-Defrawy, Boegman et al. 1986, Garthwaite and Garthwaite 1987, see review by Maes, Yirmyia et al. 2009). Furthermore, 3HK and 3HAA have been shown to exert negative effects, such as apoptosis, neuronal damage and oxidative stress throughout the generation of reactive oxygen species (Mackay, Forrest et al. 2006). KYNA acts as a potentially neuroprotective product due to its antagonist affinity for NMDA receptors (Perkins and Stone 1982). On the other hand, increased levels of KYNA have also been associated with cognitive deficits in schizophrenia patients, which may be related to disturbed glutamatergic neurotransmission. A study showed that infusion of nanomolar concentrations of KYNA, or production in rats in situ, reduced extracellular glutamate in the prefrontal cortex by inhibition of $\alpha 7$ nicotinic acetylcholine receptors ( $\alpha 7 A C h R)(W u$, Pereira et al. 2010). When cortisol is increased (like in case of stress), TDO is stimulated by GCs, resulting in increased kynurenine levels, the metabolism of which also occurs extra-hepatically. In addition, KMO is stimulated by pro-inflammatory cytokines. Therefore, in inflammation, kynurenine is further metabolised to 3HK (and 
subsequently QUIN), and this arm of the pathway is favoured over the formation of KYNA. However, next to increased levels of QUIN levels, KYNA levels are higher than normally as well, which may help to neutralise adverse effects of QUIN (see review by Myint 2012). Interestingly, in the brain, the increased metabolism of tryptophan may also result in a deficit in $5-\mathrm{HT}$, which, in case of inflammation, is not only metabolised by monoamine oxidase (MAO), but is also degraded by activated IDO (Pertz and Back 1988). Thus, IDO plays an important role in the regulation of 5-HT related mood states and the regulation of cellular immune responses (Stastny, Konstantinidis et al. 2003). As such, the kynurenine pathway connects the 5-HT and the inflammatory hypotheses in depression (Mackay, Forrest et al. 2009). In an inflammatory state, more kynurenine is available to be transported into the brain, and its metabolism in the brain is increased. The resulting metabolites of the kynurenine pathway directly affect the balance between neuroprotective and neurodegenerative processes in the brain (see review by Myint 2012). When microglia are activated, QUIN and 3HAA are excessively synthesised and astrocytes are not capable to metabolise the excess of QUIN, resulting in a loss of astrocytes due to the apoptotic proeffect of 3HK (see review by Leonard and Myint 2009). With the imbalance in those metabolites, neurons are primary exposed to the neurodegenerative effects of QUIN, which together with a decreased neuronal repair, results in a variety of structural and functional abnormalities in the brain (see review by Myint 2012). 
Some studies have examined monoamine levels in offspring after prenatal exposure to an infectious agent. Administration of Poly I:C during pregnancy in both rats and mice led to reduced hippocampal 5-HT levels in adult offspring (Winter, Djodari-Irani et al. 2009, Ohkawara, Katsuyama et al. 2015). Studies show contradictory results about dopamine levels in offspring after exposure to an infection during pregnancy. One the one hand, elevated dopamine and 3,4-Dihydroxyphenylacetic acid (DOPAC) were found in the the lateral globus pallidus and prefrontal cortex in offspring exposed to maternal Poly I:C, but no significant change was observed in striatal regions (Winter, Djodari-Irani et al. 2009). One the other hand, another study showed increased DOPAC in the offspring striatum (Ozawa, Hashimoto et al. 2006). To date, only one study has analysed the metabolites of the kynurenine pathway in both control and offspring exposed to maternal infection reporting elevated serum QUIN and reduced KYNA and kynurenine in preadolescence rat offspring exposed to prenatal maternal Poly I:C (Zavitsanou, Lim et al. 2014).

Potentially, the kynurenine pathway may represent a key player linking various processes known to be dysregulated in psychiatric disorders. As commented above, as several enzymes intervene in the production of QUIN and KYNA, the complex interaction between the various enzymes, neurotransmitters and the production of different metabolites still needs to be elucidated. 


\subsubsection{Neurodegeneration hypothesis of depression}

Recently, the "neurodegeneration hypothesis of depression" was proposed, by integrating the above mentioned concepts, the kynurenine pathway and its connection with cytokines, kynurenine metabolites, 5-HT as well as $\mathrm{N}$-methyl-D-aspartate glutamate (NMDA)-glutamatergic neurotransmission (Myint and Kim 2003). In the end, various effects of cytokines can result in either neuroprotection or neurodegeneration. Generally, neurodegeneration is preceded by inflammation (see review by Camacho-Arroyo, Lopez-Griego et al. 2009). Although some studies indicate that inflammatory reactions in the CNS are usually considered to have neurotoxic effects, evidence suggests that they can also be beneficial (Hohlfeld, Kerschensteiner et al. 2007), such as in case of tissue repair and phagocytosis of apoptotic cells and debris (see review by Ramesh, MacLean et al. 2013). In an in vivo study, Schwartz and colleagues found that Th1 cytokines can produce neuroprotective and regenerated actions (Kipnis, Mizrahi et al. 2002) as Th2 does, but to a lesser extent. They introduced the term "protective autoimmunity" which deals with the beneficial side of immune cell filtration of the CNS. Various cytokines, generally depending on their concentration, exert both neurotrophic and neurotoxic effects (see review by Rothwell and Hopkins 1995).

Pregnancy is dominated by a Th2-type response, whereas a Th-1 response can result in foetal loss (Wegmann, Lin et al. 1993, Raghupathy 1997). Lesions in CNS could lead to a systemic 
immunosuppression if they result in a shift to a Th2 cytokine pattern. It is reported that after treatment with Th2 inducers, there is an elevation in neuroprotection and regeneration. Therefore, it has been proposed that to repair the CNS after injury completely, a part of the response includes a shift to Th2, although this shift may suppress the cellular immune response and could facilitate the predisposition to an infection (see review by Hendrix and Nitsch 2007).

Altogether, in depressive disorders, the overproduction of cytokines may disturb the loop of regulation within the neuro-endocrineimmune system and hence, resulting in an acute phase response, an hyperactive HPA axis and dysregulation of 5-HT turnover (see review by Corcos, Guilbaud et al. 2002).

\section{Stress, stress related disorders, pregnancy and the immune system}

Chronic and/or excessive stress has been related to several disorders including depression (Raison, Capuron et al. 2006). Interestingly, evidence shows that stress has an impact upon the immune system (see review by Segerstrom and Miller 2004), the effects of which vary depending on the duration of the stressor. Whereas acute stress upregulates the immune response, chronic stress suppresses it (Dhabhar and McEwen 1997). An increase of lymphocites, NK cells and cytotoxic T cells has been observed after acute stress (Naliboff, Benton et al. 1991, Herbert, Cohen et al. 1994), 
while chronic stress resulted in a decrease in their number (Herbert and Cohen 1993). In several ways, exposure to stress leads to similar changes as those caused by an infection. As shown in the previous sections, exposure to infectious agents during pregnancy produces changes in GCs, 5-HT and neurotrophins, among others. In this section, we focus on the effects of stress on these features and more specifically, on the effects of stress exposure during pregnancy, showing that prenatal maternal stress and infection may have similar consequences in the offspring. For instance, dysregulation of the HPA axis and the concomitant release of GCs can both be induced by stress (see review by Levy and Tasker 2012) and by the induction of cytokines after exposure to infectious agents (see review by Silverman, Pearce et al. 2005). Acute stress results in a transient increase in GCs that activate receptors in the pituitary and hypothalamus. Thus, decreasing the release of CRF, ACTH and GCs. In case of chronic stress, a hypersecretion of GCs is produced together with a sustained activation of the central and peripheral sympathetic nervous system as a result of the desensitisation of the central GR. Subsequently, the impaired negative feedback regulation induces a chronic hypersecretion of cortisol (Leonard 2006). Another example is the availability of $5-\mathrm{HT}$, which is also affected by hypersecretion of GCs. Acute stress provokes an increased turnover of 5-HT, which is connected with an induction of tryptophan hydroxylase activity (enzyme involved in the synthesis of 5-HT) (Davis, Stanford et al. 1995). However, chronic stress causes a reduction in 5-HT turnover via stimulation of tryptophan 2,3-dioxygenase in the liver and the consequent enhanced metabolism of tryptophan throughout kynurenine pathway (Leonard 2006). 
Consequently, the $5 \mathrm{HT} 1 \mathrm{~A}$ receptor is also affected by the availability of 5$H T$, being increased its response in acute stress and decreased under chronic stress conditions. Under chronic stress conditions, $5 \mathrm{HT} 1 \mathrm{~A}$ receptors in the hippocampus are further inhibited by GCs (Chaouloff 1995, Meijer and de Kloet 1998).

In order to study the effects of prenatal stress in the offspring, several animal studies have been conducted exposing the pregnant dam to stress and, subsequently, examining the effects in the offspring. As an example, offspring exposed to prenatal stress showed an increased CRF release and levels in the amygdala (Cratty, Ward et al. 1995). In another study, Huang and colleagues subjected pregnant dams to stress and they also found increased corticosterone and CRF levels in the offspring. Moreover, 5-HT levels were increased in the hippocampus and hypothalamus of foetuses exposed to prenatal stress, whereas only hippocampal 5-HIAA levels were lower compared to control foetuses. In addition, 5-HT1A receptor levels were significantly reduced in the hippocampus and levels of the serotonin transporter (SERT) were lower in both the hippocampus and hypothalamus of foetuses exposed to prenatal stress (Huang, Xu et al. 2012). Regarding neurotrophic factors, for example, reduced brain BDNF content was observed just after birth in prenatally stressed offspring (Van den Hove, Steinbusch et al. 2006). Changes in cytokines in the offspring have also been investigated after prenatal stress showing an increased expression of IL-1B and TNF- $\alpha$ in the hippocampus and increased percentage of reactive microglia cells in CA1 compared to non-stressed offspring (Diz-Chaves, Astiz et al. 2013). However, evidence is lacking when analyzing the involvement 
of the kynurenine pathway in prenatally stressed offspring. Only in 1990, a study reported increased brain tryptophan levels in foetus exposed to prenatal stress (Peters 1990).

To sum up, exposure to stress or infection, in particular during pregnancy, may lead to similar changes in the immune system and in GCs, 5-HT and neurotrophines, among others, although the exact functional link between prenatal maternal infection and stress remains to be elucidated.
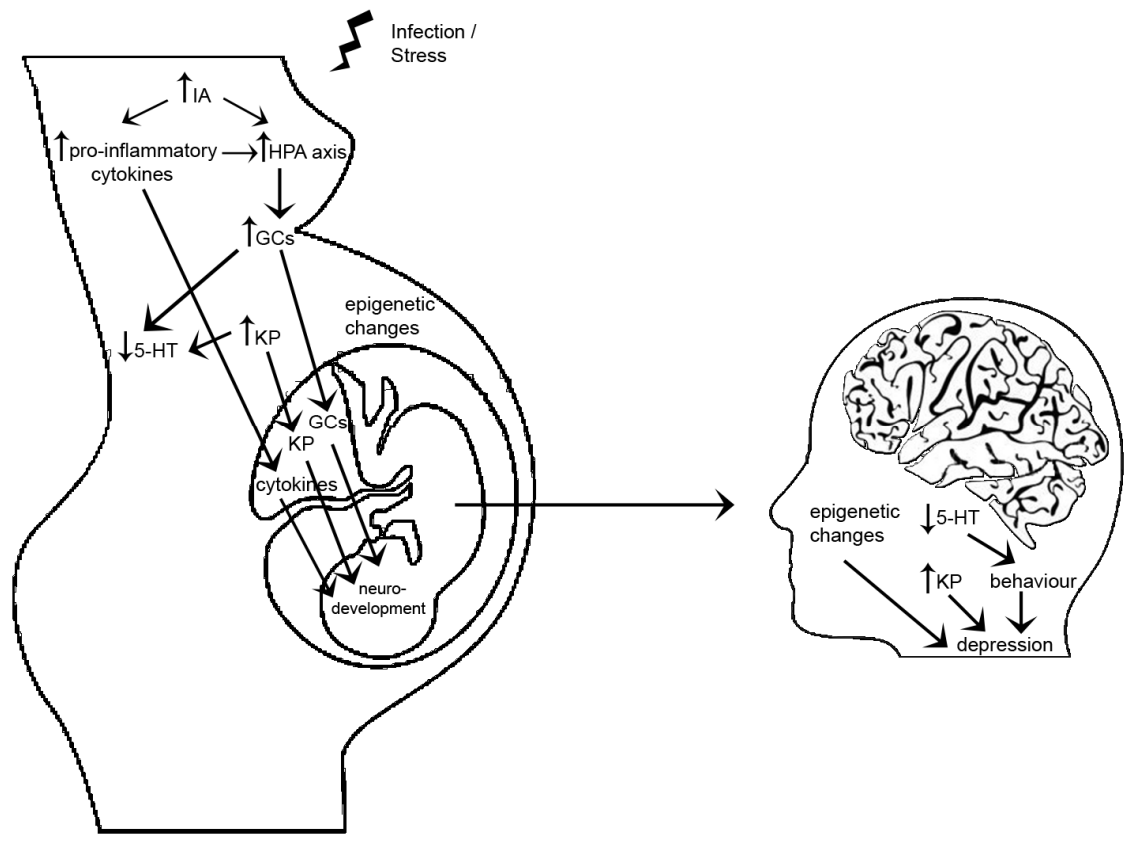

Figure 1. Integrated view of the potential mechanisms underlying the relationship between prenatal maternal infection and stress on adult psychopathology, particularly depression.

The diagram shows that exposure to an infection or stress during the preganacy results in increased immune activity. This response leads to an increase in pro-inflammatory cytokines, activation of the kynurenine pathway and the hypothalamic pituitary adrenal axis, concomitant with an increase in glucocorticoids levels in both the mother and the foetus. As a consequence, a decrease in serotonin is being induced. Glucocorticoids and cytokines can cross the blood brain barrier, whereas the activation of the kynurenine pathway in the foetus may also affect neurodevelopment in the offspring. Adult offspring prenatally exposed to infection or stress showed reduced serotonin and disturbed behaviour, which together with epigenetic changes and enhanced kynurenine pathway may lead to the development of depression. Abbreviations: IA, immune activation; HPA, hypothalamic pituitary adrenal; GCs, glucocorticoids; 5-HT, serotonin; KP, kynurenine pathway. 


\section{Discussion}

4.1 Gender differences (offspring psychopathology in response to prenatal maternal infection)

Mental disorders affect men and women differently but the exact mechanisms that cause this distinction remain unknown. For instance, the prevalence of depression in women has been reported to be 1.7 to 2.7 times higher than in men (see review by MacQueen and Chokka 2004). Most likely, a combination of various factors, such as hormonal fluctuations to which women are subjected -which are known to influence neurotransmitters- accounts for this gender variation (see review by Vigod and Stewart 2009). Furthermore, the modulatory function of those hormones and their interaction with other hormonal systems such as the HPA axis might increase the vulnerability to depression predominantly in women (see review by Steiner, Dunn et al. 2003). Interestingly, placental 11ßHSD2 activity has been reported to be reduced in case of females foetuses after inflammation, whereas in male foetuses an increase in 11ßHSD2 activity has been observed (Murphy, Gibson et al. 2003, see review by Howerton and Bale 2012), thereby particularly exposing female foetuses at risk to elevated GC exposure. Even during early CNS development, the interaction between neuroendocrine and neuroimmune pathways mediates the establishment of sex differences (Bale 2009). Immune cells express receptors for sex hormones and, as such, are also modulated by gonadal hormones (Klein, Roberts et al. 2010). It is already known that the immune response differs between males and females (see review by Fish 
2008). For instance, women show an increased immune response after exposure to viral infections (Klein and Huber 2010). Specifically, the Influenza virus has been shown to provoke an excessive proinflammatory cytokine response (de Jong, Simmons et al. 2006), which was more pronounced in females compared to males (see review by Klein, Hodgson et al. 2012). Over the last years, there has been mounting evidence for effects in the offspring after prenatal maternal infection. However, only a limited number of studies have been conducted to analyse sex differences after prenatal infection. Lante et al. showed that prenatal LPS exposure in rats led to oxidative stress only in the hippocampus of male fetuses (Lanté, Meunier et al. 2007). Another group examined the behavioural effects after prenatal exposure to LPS. Male offspring showed stronger anxiety-driven reactions and impaired spatial learning and memory (Chlodzinska, Gajerska et al. 2011). Moreover, male offspring exposed prenatally to LPS showed elevated numbers of macrophage and infiltrating cells and incremented loss of hippocampal volume (Dada, Rosenzweig et al. 2014). However, another study using prenatal exposure to LPS reported altered behaviour especially in females, such as sensorimotor impairment (Wang, Meng et al. 2010). In relation to autism, a recent study showed that male offspring prenatally exposed to both LPS and Poly I:C showed increased repetitive behaviour. In addition, decreased motor activity was observed only in male offspring from Poly I:C challenged dams (Xuan and Hampson 2014). A study focussing on schizophrenia-related phenotypes showed different effects after prenatal infection between male and female as well. Altered PPI was 
reported in both pubertal and adult males, but only in pubertal females after LPS injection to the pregnant rat. In addition, males showed impaired recognition memory for objects to a greater extent. Finally, in male offspring prenatally exposed to LPS, a more pronounced reduction of myelination in cortical and limbic brain regions and decreased parvalbumin-expressing cells in the medial prefrontal cortex, hippocampus and entorhinal cortex has been observed compared to females (Wischhof, Irrsack et al. 2015).

Altogether, it is important to consider sex differences when assessing a subject's response to neuroendocrine or immune challenges early in life, such as prenatal infection or stress, and how such perturbations might then program future responses to similar challenges (Bale 2009).

\section{$\underline{4.2 \text { Epigenetics }}$}

Recently, the effects of epigenetic marks in the nervous system have been raised the attention of researchers. Epigenetic modifications are heritable changes in gene expression that do not involve alterations of the DNA sequence. The most-studied epigenetic mechanisms to date are DNA methylation, histone modifications and noncoding RNA expression. Diverse functions in the nervous system are under the influence of epigenetic mechanisms, such as neuronal-glial differentiation, modulation of neural plasticity and behaviour, adult neurogenesis, memory and cognition (see review by Ravi and Kannan 2013). Furthermore, prenatal infection is reported to produce epigenetic modifications of CNS genes, resulting in altered structure or function of 
specific neuronal pathways (see review by Boksa 2010). It is already known that in response to a microbial exposure, cells exert modifications in the transcriptional program of specific genes in order to induce a competent response. However, pathogens have been shown to interrupt this response to ensure an adequate environment for their survival (Hamon, Batsché et al. 2007). Thus, the target host genes for epigenetic control are genes involved in cell cycle progression, survival, inflammation and immunity (Al Akeel 2013). The activation or silencing of genes is produced by transcription factors as well as epigenetic modulators (see review by Bierne, Hamon et al. 2012), and both of them have been shown to be altered by pathogens. More specifically, histone modifications are the main epigenetic processes affected by bacteria. One example is the increase in histone acetylation and phosphorylation, specifically at the promoter of IL-8 in dendritic cells, after LPS exposure (Saccani, Pantano et al. 2002). Recent evidence suggest that the regulation of histone acetylation, by directly modifying acetylation or through impact upon histone deacetylase (HDAC) activity, on host defense genes may be a common characteristic of bacterial infections (see review by Garcia-Garcia, Barat et al. 2009, Paschos and Allday 2010). For instance, inhibition of histone 3 acetylation and phosphorylation impairs the host innate immune response. But also changes in the expression of HDACs have been shown, resulting in silencing of host defense genes (see review by Bierne, Hamon et al. 2012). In addition, DNA methyltransferase (DNMT) expression has been also found to be increased (Al Akeel 2013). Several viruses have been shown to modulate 
epigenetic mechanisms. For instance, increased expression of DNMTs is found in hepatitis B virus-infected cells (Vivekanandan, Daniel et al. 2010), whereas DNA hypermethylation has been associated with the presence of human papillomavirus (Katargin, Pavlova et al. 2009). But also histones are modulated by virus pathogens, such as the adenovirus, which reduces acetylation of histone 3 (Horwitz, Zhang et al. 2008). With respect to pregnancy infection, only a limited number of studies have been conducted to analyze the epigenetic changes in the offspring exposed to prenatal infection. One example shows reduced DNA methylation in the hypothalamus of adolescent murine offspring, after prenatal Poly I:C acid exposure (Basil, Li et al. 2014). In another study, exposing pregnant mice to Poly $\mathrm{I}: \mathrm{C}$ was associated with global hypoacetylation of histone $\mathrm{H} 3$ and histone $\mathrm{H} 4$ in the cortex of juvenile offspring (Tang, Jia et al. 2013).

All in all, infections can modulate epigenetic processes in many ways (see review by Bierne, Hamon et al. 2012). Epigenetic reprogramming is being employed by cells as an adaptive mechanism to ensure a favorable metabolic status after exposure to physiological substances or infectious agents (Waly, Hornig et al. 2012). As mentioned above, epigenetic regulation plays an important role in the development and differentiation of the nervous system that may serve to explain the molecular basis of the CNS response in case of injury after exposure to infection. Clearly, the magnitude and exact involvement of epigenetic modifications in mediating changes in brain function and behaviour in response to prenatal maternal infection remains to be disclosed. 


\subsection{Treatment options}

Many types of treatments have been developed in order to alleviate the symptoms of depression. However, to date, the overall efficacy of current treatment strategies is limited and still a considerable number of patients show recurrence (see review by Burcusa and lacono 2007). Examples of common treatments applied are psychotherapy, antidepressants and electroconvulsive therapy (ECT), among others. Currently, antidepressants, such as selective serotonin reuptake inhibitors (SSRIs), serotonin and norepinephrine reuptake inhibitors (SNRIs) and monoamine oxidase (MAO) inhibitors, in combination with psychotherapy are the preferred choice. However, as a consequence of their low efficacy in some patients (Nelson 1998, Fournier, DeRubeis et al. 2010), new strategies have been investigated, such as HDAC inhibitors. HDAC inhibitors are involved in transcriptional regulation of cytokines, in immunologic signaling pathways, and inflammatory responses (see review by Qureshi and Mehler 2011). On the one hand, the commonly used HDAC inhibitor valproic acid (VPA), is found to dedifferentiate committed oligodendrocytes precursors to multipotent neural progenitors, thus inducing neuronal differentiation (Liu, Han et al. 2007). On the other hand, HDAC inhibitors are known to produce adverse side effects. Thus, their use in psychiatric disorders is still under discussion. 


\subsection{Summary and outlook}

This review addressed the possible mechanisms through immune activation during pregnancy may lead to development of psychiatric disorders in the offspring. Infections by bacteria or other virus during pregnancy increase the risk for the development of mental disorders in the offspring. Among others, cytokines, associated with a pathogenic immune response are thought to impact upon foetal brain development. It is still unknown, however, how exactly viral or bacterial exposure leads to pathology in the CNS (Zuckerman and Weiner 2005). Many experiments in mice and rats have provided evidence about abnormalities in brain development and structure in offspring after prenatal exposure to pathogens, such as synthetic double-stranded RNA Poly I:C, which is reported to provoke a decrease in hippocampal function in adulthood (Zuckerman, Rehavi et al. 2003). Moreover, epigenetic processes may represent an important player in mediating the molecular changes that may contribute to the development of psychiatric disorders.

Further studies should be done to understand how prenatal environmental insults can affect neuropathological and psychopathological brain development of offspring and to develop preventive strategies. A detailed analysis of the kynurenine pathway (metabolites and enzymes) could be very helpful in order to improve diagnose and treatment of psychiatric disorders. 


\section{$\underline{\text { References }}$}

Al Akeel, R. (2013). "Role of epigenetic reprogramming of host genes in bacterial pathogenesis." Saudi J Biol Sci 20(4): 305-309.

Amirchaghmaghi, E., S. A. Taghavi, F. Shapouri, S. Saeidi, A. Rezaei and R. Aflatoonian (2013). "The role of toll like receptors in pregnancy." Int J Fertil Steril 7(3): 147-154.

Ashdown, H., Y. Dumont, M. Ng, S. Poole, P. Boksa and G. N. Luheshi (2006). "The role of cytokines in mediating effects of prenatal infection on the fetus: implications for schizophrenia." Mol Psychiatry 11(1): 47-55.

Asiaei, M., J. Solati and A.-A. Salari (2011). "Prenatal exposure to Ips leads to longlasting physiological consequences in male offspring." Developmental Psychobiology 53(8): 828-838.

Atladottir, H. O., P. Thorsen, L. Ostergaard, D. E. Schendel, S. Lemcke, M. Abdallah and E. T. Parner (2010). "Maternal infection requiring hospitalization during pregnancy and autism spectrum disorders." J Autism Dev Disord 40(12): 1423-1430.

Bale, T. L. (2009). "Neuroendocrine and Immune Influences on the CNS: It's a Matter of Sex." Neuron 64(1): 13-16.

Basil, P., Q. Li, E. L. Dempster, J. Mill, P. C. Sham, C. C. Wong and G. M. McAlonan (2014). "Prenatal maternal immune activation causes epigenetic differences in adolescent mouse brain." Transl Psychiatry 4: e455.

Bay-Richter, C., S. Janelidze, L. Hallberg and L. Brundin (2011). "Changes in behaviour and cytokine expression upon a peripheral immune challenge." Behavioural brain research 222(1): 193-199.

Benros, M. E., B. L. Waltoft, M. Nordentoft, S. D. Ostergaard, W. W. Eaton, J. Krogh and P. B. Mortensen (2013). "Autoimmune diseases and severe infections as risk factors for mood disorders: a nationwide study." JAMA Psychiatry 70(8): 812-820.

Bierne, H., M. Hamon and P. Cossart (2012). "Epigenetics and bacterial infections." Cold Spring Harb Perspect Med 2(12): a010272.

Boksa, P. (2010). "Effects of prenatal infection on brain development and behavior: a review of findings from animal models." Brain Behav Immun 24(6): 881897.

Borrell, J., J. M. Vela, A. Arevalo-Martin, E. Molina-Holgado and C. Guaza (2002). "Prenatal immune challenge disrupts sensorimotor gating in adult rats. Implications for the etiopathogenesis of schizophrenia." Neuropsychopharmacology 26(2): 204-215.

Brown, A. S. (2006). "Prenatal infection as a risk factor for schizophrenia." Schizophrenia bulletin 32(2): 200-202. 
Bulmer, J. N., D. Pace and A. Ritson (1988). "Immunoregulatory cells in human decidua: morphology, immunohistochemistry and function." Reprod Nutr Dev 28(6B): 1599-1613.

Burcusa, S. L. and W. G. Iacono (2007). "Risk for recurrence in depression." Clin Psychol Rev 27(8): 959-985.

Camacho-Arroyo, I., L. Lopez-Griego and J. Morales-Montor (2009). "The role of cytokines in the regulation of neurotransmission." Neuroimmunomodulation 16(1): 1-12.

Carta, M., M. Hardoy, A. Garofalo, E. Pisano, V. Nonnoi, G. Intilla, G. Serra, C. Balestrieri, L. Chessa, C. Cauli, M. Lai and P. Farci (2007). "Association of chronic hepatitis $\mathrm{C}$ with major depressive disorders: irrespective of interferon-alpha therapy." Clinical Practice and Epidemiology in Mental Health 3(1): 22.

Chaouloff, F. (1995). "Regulation of 5-HT receptors by corticosteroids: where do we stand?" Fundamental \& clinical pharmacology 9(3): 219-233.

Chlodzinska, N., M. Gajerska, K. Bartkowska, K. Turlejski and R. L. Djavadian (2011). "Lipopolysaccharide injected to pregnant mice affects behavior of their offspring in adulthood." Acta Neurobiol Exp (Wars) 71(4): 519-527.

Coppen, A. (1967). "The biochemistry of affective disorders." Br J Psychiatry 113(504): 1237-1264.

Corcos, M., O. Guilbaud, L. Hjalmarsson, J. Chambry and P. Jeammet (2002). "Cytokines and depression: an analogic approach." Biomed Pharmacother 56(2): 105-110.

Cratty, M. S., H. E. Ward, E. A. Johnson, A. J. Azzaro and D. L. Birkle (1995). "Prenatal stress increases corticotropin-releasing factor (CRF) content and release in rat amygdala minces." Brain Res 675(1-2): 297-302.

Dada, T., J. M. Rosenzweig, M. Al Shammary, W. Firdaus, S. Al Rebh, T. Borbiev, A. Tekes, J. Zhang, E. Alqahtani, S. Mori, M. V. Pletnikov, M. V. Johnston and I. Burd (2014). "Mouse model of intrauterine inflammation: Sex-specific differences in long-term neurologic and immune sequelae." Brain, Behavior, and Immunity 38(0): 142-150.

Dang, Y., W. E. Dale and O. R. Brown (2000). "Comparative effects of oxygen on indoleamine 2,3-dioxygenase and tryptophan 2,3-dioxygenase of the kynurenine pathway." Free Radic Biol Med 28(4): 615-624.

Dantzer, R., J. C. O'Connor, G. G. Freund, R. W. Johnson and K. W. Kelley (2008). "From inflammation to sickness and depression: when the immune system subjugates the brain." Nat Rev Neurosci 9(1): 46-56.

Davis, S., S. C. Stanford and D. Heal (1995). "Long-lasting effects of an acute stress on the neurochemistry and function of 5-hydroxytryptaminergic neurones in the mouse brain." Psychopharmacology 118(3): 267-272. 
de Jong, M. D., C. P. Simmons, T. T. Thanh, V. M. Hien, G. J. Smith, T. N. Chau, D. M. Hoang, N. V. Chau, T. H. Khanh, V. C. Dong, P. T. Qui, B. V. Cam, Q. Ha do, Y. Guan, J. S. Peiris, N. T. Chinh, T. T. Hien and J. Farrar (2006). "Fatal outcome of human influenza $A(\mathrm{H} 5 \mathrm{~N} 1)$ is associated with high viral load and hypercytokinemia." Nat Med 12(10): 1203-1207.

Dekel, N., Y. Gnainsky, I. Granot and G. Mor (2010). "Inflammation and implantation." Am J Reprod Immunol 63(1): 17-21.

Dhabhar, F. S. and B. S. McEwen (1997). "Acute stress enhances while chronic stress suppresses cell-mediated immunity in vivo: a potential role for leukocyte trafficking." Brain Behav Immun 11(4): 286-306.

Diz-Chaves, Y., M. Astiz, M. J. Bellini and L. M. Garcia-Segura (2013). "Prenatal stress increases the expression of proinflammatory cytokines and exacerbates the inflammatory response to LPS in the hippocampal formation of adult male mice." Brain Behav Immun 28: 196-206.

Dunn, A. J. (1988). "Systemic interleukin-1 administration stimulates hypothalamic norepinephrine metabolism parallelling the increased plasma corticosterone." Life Sci 43(5): 429-435.

Dunn, A. J., A. H. Swiergiel and R. de Beaurepaire (2005). "Cytokines as mediators of depression: what can we learn from animal studies?" Neurosci Biobehav Rev 29(4-5): 891-909.

Edwards, C. R. W., R. Benediktsson, R. S. Lindsay and J. R. Seckl (1993). "Dysfunction of placental glucocorticoid barrier: link between fetal environment and adult hypertension?" The Lancet 341(8841): 355-357.

el-Defrawy, S. R., R. J. Boegman, K. Jhamandas and R. J. Beninger (1986). "The neurotoxic actions of quinolinic acid in the central nervous system." Canadian journal of physiology and pharmacology 64(3): 369-375.

Enayati, M., J. Solati, M.-H. Hosseini, H.-R. Shahi, G. Saki and A.-A. Salari (2012). "Maternal infection during late pregnancy increases anxiety- and depression-like behaviors with increasing age in male offspring." Brain Research Bulletin 87(2-3): 295-302.

Fernstrom, J. D. (1984). "Tryptophan availability and serotonin synthesis in rat brain: effects of experimental diabetes." H.G. Schlossberger, W. Kochen, B. Linzen, H. Steinhart (Eds.), Progress in tryptophan and serotonin research, Walter de Gruyter, Berlin (1984), pp. 161-172.

Fish, E. N. (2008). "The X-files in immunity: sex-based differences predispose immune responses." Nat Rev Immunol 8(9): 737-744.

Fournier, J. C., R. J. DeRubeis, S. D. Hollon, S. Dimidjian, J. D. Amsterdam, R. C. Shelton and J. Fawcett (2010). "Antidepressant drug effects and depression severity: a patient-level meta-analysis." Jama 303(1): 47-53. 
Gál, E. M. and A. Sherman (1980). "I-Kynurenine Its synthesis and possible regulatory function in brain." Neurochemical Research 5(3): 223-239.

Garcia-Garcia, J. C., N. C. Barat, S. J. Trembley and J. S. Dumler (2009). "Epigenetic silencing of host cell defense genes enhances intracellular survival of the rickettsial pathogen Anaplasma phagocytophilum." PLoS Pathog 5(6): e1000488.

Garthwaite, G. and J. Garthwaite (1987). "Quinolinate mimics neurotoxic actions of $\mathrm{N}$-methyl-D-aspartate in rat cerebellar slices." Neurosci Lett 79(1-2): 35-39.

Gibney, S. M. and H. A. Drexhage (2013). "Evidence for a dysregulated immune system in the etiology of psychiatric disorders." $\underline{\mathrm{J} \text { Neuroimmune Pharmacol }}$ 8(4): 900-920.

Gilmore, J. H., L. F. Jarskog and S. Vadlamudi (2003). "Maternal infection regulates BDNF and NGF expression in fetal and neonatal brain and maternal-fetal unit of the rat." Journal of Neuroimmunology 138(1-2): 49-55.

Golan, H. M., V. Lev, M. Hallak, Y. Sorokin and M. Huleihel (2005). "Specific neurodevelopmental damage in mice offspring following maternal inflammation during pregnancy." Neuropharmacology 48(6): 903-917.

Hamon, M. A., E. Batsché, B. Régnault, T. N. Tham, S. Seveau, C. Muchardt and P. Cossart (2007). "Histone modifications induced by a family of bacterial toxins." Proceedings of the National Academy of Sciences of the United States of America 104(33): 13467-13472.

Hashmi, A. M., M. A. Aftab, N. Mazhar, M. Umair and Z. Butt (2013). "The fiery landscape of depression: A review of the inflammatory hypothesis." Pakistan journal of medical sciences 29(3): 877.

Hayaishi, O. (1976). "Properties and function of indoleamine 2,3-dioxygenase." $\underline{\mathrm{J}}$ Biochem 79(4): 13P-21P.

Hendrix, S. and R. Nitsch (2007). "The role of T helper cells in neuroprotection and regeneration." J Neuroimmunol 184(1-2): 100-112.

Herbert, T. B. and S. Cohen (1993). "Stress and immunity in humans: a meta-analytic review." Psychosom Med 55(4): 364-379.

Herbert, T. B., S. Cohen, A. L. Marsland, E. A. Bachen, B. S. Rabin, M. F. Muldoon and S. B. Manuck (1994). "Cardiovascular reactivity and the course of immune response to an acute psychological stressor." Psychosom Med 56(4): 337344.

Hidaka, Y., N. Amino, Y. Iwatani, T. Kaneda, N. Mitsuda, Y. Morimoto, O. Tanizawa and K. Miyai (1991). "Changes in natural killer cell activity in normal pregnant and postpartum women: increases in the first trimester and postpartum period and decrease in late pregnancy." Journal of Reproductive Immunology 20(1): 73-83. 
Hohlfeld, R., M. Kerschensteiner and E. Meinl (2007). "Dual role of inflammation in CNS disease." Neurology 68(22 Suppl 3): S58-63; discussion S91-56.

Horwitz, G. A., K. Zhang, M. A. McBrian, M. Grunstein, S. K. Kurdistani and A. J. Berk (2008). "Adenovirus Small ela Alters Global Patterns of Histone Modification." Science 321(5892): 1084-1085.

Howerton, C. L. and T. L. Bale (2012). "Prenatal programing: at the intersection of maternal stress and immune activation." Horm Behav 62(3): 237-242.

Huang, Y., H. Xu, H. Li, H. Yang, Y. Chen and X. Shi (2012). "Pre-gestational stress reduces the ratio of 5-HIAA to 5-HT and the expression of 5-HT1A receptor and serotonin transporter in the brain of foetal rat." BMC Neuroscience 13(1): 22.

Irwin, M. (2002). "Psychoneuroimmunology of depression: clinical implications." Brain Behav Immun 16(1): 1-16.

Jiang, P., T. Zhu, W. Zhao, J. Shen, Y. Yu, J. Xu, X. Chen and H. Yu (2013). "The persistent effects of maternal infection on the offspring's cognitive performance and rates of hippocampal neurogenesis." Progress in NeuroPsychopharmacology and Biological Psychiatry 44(0): 279-289.

Karege, F., G. Perret, G. Bondolfi, M. Schwald, G. Bertschy and J.-M. Aubry (2002). "Decreased serum brain-derived neurotrophic factor levels in major depressed patients." Psychiatry Research 109(2): 143-148.

Katargin, A. N., L. S. Pavlova, F. L. Kisseljov and N. P. Kisseljova (2009). "Hypermethylation of genomic 3.3-kb repeats is frequent event in HPVpositive cervical cancer." BMC Med Genomics 2: 30.

Kerschensteiner, M., C. Stadelmann, G. Dechant, H. Wekerle and R. Hohlfeld (2003). "Neurotrophic cross-talk between the nervous and immune systems: implications for neurological diseases." Ann Neurol 53(3): 292-304.

Kipnis, J., T. Mizrahi, E. Yoles, A. Ben-Nun and M. Schwartz (2002). "Myelin specific Th1 cells are necessary for post-traumatic protective autoimmunity." J Neuroimmunol 130(1-2): 78-85.

Kirsten, T. B., L. L. Lippi, E. Bevilacqua and M. M. Bernardi (2013). "LPS Exposure Increases Maternal Corticosterone Levels, Causes Placental Injury and Increases IL-1B Levels in Adult Rat Offspring: Relevance to Autism." PLoS ONE 8(12): e82244.

Kitraki, E., C. Kittas and F. Stylianopoulou (1997). "Glucocorticoid receptor gene expression during rat embryogenesis. An in situ hybridization study." Differentiation 62(1): 21-31.

Klein, S. L., A. Hodgson and D. P. Robinson (2012). "Mechanisms of sex disparities in influenza pathogenesis." J Leukoc Biol 92(1): 67-73.

Klein, S. L. and S. Huber (2010). Sex differences in susceptibility to viral infection. Sex hormones and immunity to infection, Springer: 93-122. 
Klein, S. L., C. Roberts, S. Kovats, E. Carreras and H. Agrawal (2010). Sex Steroid Receptors in Immune Cells. Sex Hormones and Immunity to Infection, Springer Berlin Heidelberg: 53-91.

Klimek, V., C. Stockmeier, J. Overholser, H. Y. Meltzer, S. Kalka, G. Dilley and G. A. Ordway (1997). "Reduced levels of norepinephrine transporters in the locus coeruleus in major depression." J Neurosci 17(21): 8451-8458.

Kronfol, Z. and D. G. Remick (2000). "Cytokines and the brain: implications for clinical psychiatry." Am J Psychiatry 157(5): 683-694.

Lanté, F., J. Meunier, J. Guiramand, T. Maurice, M. Cavalier, M.-C. de Jesus Ferreira, R. Aimar, C. Cohen-Solal, M. Vignes and G. Barbanel (2007). "Neurodevelopmental damage after prenatal infection: Role of oxidative stress in the fetal brain." Free Radical Biology and Medicine 42(8): 12311245.

Leonard, B. E. (2006). "HPA and immune axes in stress: involvement of the serotonergic system." Neuroimmunomodulation 13(5-6): 268-276.

Leonard, B. E. and A. Myint (2009). "The psychoneuroimmunology of depression." Hum Psychopharmacol 24(3): 165-175.

Levy, B. H. and J. G. Tasker (2012). "Synaptic regulation of the hypothalamicpituitary-adrenal axis and its modulation by glucocorticoids and stress." Front Cell Neurosci 6: 24.

Lin, Y.-L., S.-Y. Lin and S. Wang (2012). "Prenatal lipopolysaccharide exposure increases anxiety-like behaviors and enhances stress-induced corticosterone responses in adult rats." Brain, Behavior, and Immunity 26(3): 459-468.

Lin, Y.-L. and S. Wang (2014). "Prenatal lipopolysaccharide exposure increases depression-like behaviors and reduces hippocampal neurogenesis in adult rats." Behavioural Brain Research 259(0): 24-34.

Liu, A., Y. R. Han, J. Li, D. Sun, M. Ouyang, M. R. Plummer and P. Casaccia-Bonnefil (2007). "The Glial or Neuronal Fate Choice of Oligodendrocyte Progenitors Is Modulated by Their Ability to Acquire an Epigenetic Memory." The Journal of neuroscience : the official journal of the Society for Neuroscience 27(27): 7339-7343.

Maayan, R., E. Ram, D. Biton, H. Cohen, E. Baharav, R. D. Strous and A. Weizman (2012). "The influence of DHEA pretreatment on prepulse inhibition and the HPA-axis stress response in rat offspring exposed prenatally to polyriboinosinic-polyribocytidylic-acid (PIC)." Neuroscience Letters 521(1): 6-10.

Mackay, G. M., C. M. Forrest, J. Christofides, M. A. Bridel, S. Mitchell, R. Cowlard, T. W. Stone and L. G. Darlington (2009). "Kynurenine metabolites and 
inflammation markers in depressed patients treated with fluoxetine or counselling." Clin Exp Pharmacol Physiol 36(4): 425-435.

Mackay, G. M., C. M. Forrest, N. Stoy, J. Christofides, M. Egerton, T. W. Stone and L. G. Darlington (2006). "Tryptophan metabolism and oxidative stress in patients with chronic brain injury." Eur J Neurol 13(1): 30-42.

MacQueen, G. and P. Chokka (2004). "Special Issues in the Management of Depression in Women." Canadian Journal of Psychiatry 49(1): 27S-40S.

Maes, M. (1999). "Major depression and activation of the inflammatory response system." Adv Exp Med Biol 461: 25-46.

Maes, M. (2008). "The cytokine hypothesis of depression: inflammation, oxidative $\&$ nitrosative stress (IO\&NS) and leaky gut as new targets for adjunctive treatments in depression." Neuro Endocrinol Lett 29(3): 287-291.

Maes, M. (2011). "Depression is an inflammatory disease, but cell-mediated immune activation is the key component of depression." Progress in NeuroPsychopharmacology and Biological Psychiatry 35(3): 664-675.

Maes, M., B. E. Leonard, A. M. Myint, M. Kubera and R. Verkerk (2011). "The new '5-HT' hypothesis of depression: cell-mediated immune activation induces indoleamine 2,3-dioxygenase, which leads to lower plasma tryptophan and an increased synthesis of detrimental tryptophan catabolites (TRYCATs), both of which contribute to the onset of depression." Prog Neuropsychopharmacol Biol Psychiatry 35(3): 702-721.

Maes, M., H. Y. Meltzer, S. ScharpÃ", E. n. Bosmans, E. Suy, I. De Meester, J. Calabrese and P. Cosyns (1993). "Relationships between lower plasma Ltryptophan levels and immune-inflammatory variables in depression." Psychiatry Research 49(2): 151-165.

Maes, M., S. Scharpe, E. Bosmans, M. Vandewoude, E. Suy, W. Uyttenbroeck, W. Cooreman, C. Vandervorst and J. Raus (1992). "Disturbances in acute phase plasma proteins during melancholia: Additional evidence for the presence of an inflammatory process during that illness." Progress in NeuroPsychopharmacology and Biological Psychiatry 16(4): 501-515.

Maes, M., R. Smith and S. Scharpe (1995). "The monocyte-T-lymphocyte hypothesis of major depression." Psychoneuroendocrinology 20(2): 111-116.

Maes, M., R. Yirmyia, J. Noraberg, S. Brene, J. Hibbeln, G. Perini, M. Kubera, P. Bob, B. Lerer and M. Maj (2009). "The inflammatory \& neurodegenerative (I\&ND) hypothesis of depression: leads for future research and new drug developments in depression." Metab Brain Dis 24(1): 27-53.

Marques, A. H., G. Cizza and E. Sternberg (2007). "[Brain-immune interactions and implications in psychiatric disorders]." Rev Bras Psiquiatr 29 Suppl 1: S2732. 
Martinot, M., V. Bragulat, E. Artiges, F. Dolle, F. Hinnen, R. Jouvent and J. Martinot (2001). "Decreased presynaptic dopamine function in the left caudate of depressed patients with affective flattening and psychomotor retardation." Am J Psychiatry 158(2): 314-316.

Matthews, S. G. (2000). "Antenatal Glucocorticoids and Programming of the Developing CNS." Pediatr Res 47(3): 291-300.

Meijer, O. C. and E. R. de Kloet (1998). "Corticosterone and serotonergic neurotransmission in the hippocampus: functional implications of central corticosteroid receptor diversity." Crit Rev Neurobiol 12(1-2): 1-20.

Meyer, U., M. Nyffeler, B. K. Yee, I. Knuesel and J. Feldon (2008). "Adult brain and behavioral pathological markers of prenatal immune challenge during early/middle and late fetal development in mice." Brain Behav Immun 22(4): 469-486.

Moffett, J. R., K. L. Blinder, C. N. Venkateshan and M. A. A. Namboodiri (1998). "Differential effects of kynurenine and tryptophan treatment on quinolinate immunoreactivity in rat lymphoid and non-lymphoid organs." Cell and Tissue Research 293(3): 525-534.

Mor, G. (2008). "Inflammation and pregnancy: the role of toll-like receptors in trophoblast-immune interaction." Ann N Y Acad Sci 1127: 121-128.

Mor, G. and I. Cardenas (2010). "The immune system in pregnancy: a unique complexity." Am J Reprod Immunol 63(6): 425-433.

Murphy, V. E., P. G. Gibson, W. B. Giles, T. Zakar, R. Smith, A. M. Bisits, C. G. Kessell and V. L. Clifton (2003). "Maternal Asthma Is Associated with Reduced Female Fetal Growth." American Journal of Respiratory and Critical Care Medicine 168(11): 1317-1323.

Myint, A. M. (2012). "Kynurenines: from the perspective of major psychiatric disorders." FEBS Journal 279(8): 1375-1385.

Myint, A. M. and Y. K. Kim (2003). "Cytokine-serotonin interaction through IDO: a neurodegeneration hypothesis of depression." Med Hypotheses 61(5-6): 519-525.

Naliboff, B. D., D. Benton, G. F. Solomon, J. E. Morley, J. L. Fahey, E. T. Bloom, T. Makinodan and S. L. Gilmore (1991). "Immunological changes in young and old adults during brief laboratory stress." Psychosom Med 53(2): 121-132.

Nelson, J. C. (1998). "Overcoming treatment resistance in depression." Journal of Clinical Psychiatry 59(16): 13-19.

Nemeth, H., J. Toldi and L. Vecsei (2005). "Role of kynurenines in the central and peripherial nervous systems." Current neurovascular research 2(3): 249260.

Niinisalo, P., A. Raitala, M. Pertovaara, S. S. Oja, T. Lehtimaki, M. Kahonen, A. Reunanen, A. Jula, L. Moilanen, Y. A. Kesaniemi, M. S. Nieminen and M. 
Hurme (2008). "Indoleamine 2,3-dioxygenase activity associates with cardiovascular risk factors: the Health 2000 study." Scand J Clin Lab Invest 68(8): 767-770.

Ohkawara, T., T. Katsuyama, M. Ida-Eto, N. Narita and M. Narita (2015). "Maternal viral infection during pregnancy impairs development of fetal serotonergic neurons." Brain and Development 37(1): 88-93.

Ozawa, K., K. Hashimoto, T. Kishimoto, E. Shimizu, H. Ishikura and M. Iyo (2006). "Immune activation during pregnancy in mice leads to dopaminergic hyperfunction and cognitive impairment in the offspring: a neurodevelopmental animal model of schizophrenia." Biol Psychiatry 59(6): 546-554.

Parboosing, R., Y. Bao, L. Shen, C. A. Schaefer and A. S. Brown (2013). "Gestational Influenza and Bipolar Disorder in Adult OffspringGestational Influenza and Bipolar Disorder." JAMA Psychiatry: 1-8.

Paschos, K. and M. J. Allday (2010). "Epigenetic reprogramming of host genes in viral and microbial pathogenesis." Trends Microbiol 18(10): 439-447.

Perkins, M. N. and T. W. Stone (1982). "An iontophoretic investigation of the actions of convulsant kynurenines and their interaction with the endogenous excitant quinolinic acid." Brain Research 247(1): 184-187.

Pertz, H. and W. Back (1988). "[Synthesis and resolution of chiral ring-opened serotonin analogs of the 5-hydroxykynuramine type]." Pharm Acta Helv 63(4-5): 128-131.

Peters, D. A. V. (1990). "Maternal stress increases fetal brain and neonatal cerebral cortex 5-hydroxytryptamine synthesis in rats: A possible mechanism by which stress influences brain development." Pharmacology Biochemistry and Behavior 35(4): 943-947.

Qureshi, I. A. and M. F. Mehler (2011). "Epigenetics, Nervous System Tumors, and Cancer Stem Cells." Cancers 3(3): 3525-3556.

Raghupathy, R. (1997). "Th 1-type immunity is incompatible with successful pregnancy." Immunology Today 18(10): 478-482.

Raison, C. L., L. Capuron and A. H. Miller (2006). "Cytokines sing the blues: inflammation and the pathogenesis of depression." Trends Immunol 27(1): 24-31.

Raison, C. L. and A. H. Miller (2013). "Do cytokines really sing the blues?" Cerebrum 2013: 10.

Ramesh, G., A. G. MacLean and M. T. Philipp (2013). "Cytokines and chemokines at the crossroads of neuroinflammation, neurodegeneration, and neuropathic pain." Mediators Inflamm 2013: 480739.

Ravi, B. and M. Kannan (2013). "Epigenetics in the nervous system: An overview of its essential role." Indian journal of human genetics 19(4): 384. 
Rook, G. A., R. Hernandez-Pando and S. L. Lightman (1994). "Hormones, peripherally activated prohormones and regulation of the Th1/Th2 balance." Immunol Today 15(7): 301-303.

Rothwell, N. J. and S. J. Hopkins (1995). "Cytokines and the nervous system II: Actions and mechanisms of action." Trends Neurosci 18(3): 130-136.

Saccani, S., S. Pantano and G. Natoli (2002). "p38-Dependent marking of inflammatory genes for increased NF-kappa B recruitment." Nat Immunol 3(1): 69-75.

Schildkraut, J. J. (1965). "The catecholamine hypothesis of affective disorders: a review of supporting evidence." American Journal of Psychiatry 122(5): 509-522.

Seckl, J. R. (1997). "11beta-Hydroxysteroid dehydrogenase in the brain: a novel regulator of glucocorticoid action?" Front Neuroendocrinol 18(1): 49-99.

Segerstrom, S. C. and G. E. Miller (2004). "Psychological Stress and the Human Immune System: A Meta-Analytic Study of 30 Years of Inquiry." Psychological bulletin 130(4): 601-630.

Shelton, R. C. (2006). "Management of major depressive disorder following failure of first antidepressant treatment." Primary psychiatry 13(4): 73.

Shelton, R. C. and A. H. Miller (2011). "Inflammation in depression: is adiposity a cause?" Dialogues Clin Neurosci 13(1): 41-53.

Silverman, M. N., B. D. Pearce, C. A. Biron and A. H. Miller (2005). "Immune Modulation of the Hypothalamic-Pituitary-Adrenal (HPA) Axis during Viral Infection." Viral immunology 18(1): 41-78.

Sluzewska, A. (1999). "Indicators of immune activation in depressed patients." Adv Exp Med Biol 461: 59-73.

Smith, R. S. (1991). "The macrophage theory of depression." Med Hypotheses 35(4): 298-306.

Somerset, D. A., Y. Zheng, M. D. Kilby, D. M. Sansom and M. T. Drayson (2004). "Normal human pregnancy is associated with an elevation in the immune suppressive CD25+ CD4+ regulatory T-cell subset." Immunology 112(1): 3843.

Song, C., A. Lin, S. Bonaccorso, C. Heide, R. Verkerk, G. Kenis, E. Bosmans, S. Scharpe, A. Whelan, P. Cosyns, R. de Jongh and M. Maes (1998). "The inflammatory response system and the availability of plasma tryptophan in patients with primary sleep disorders and major depression." Journal of Affective Disorders 49(3): 211-219.

Sperner-Unterweger, B., C. Miller, B. Holzner, A. Laich, B. Widner, W. W. Fleischhacker and D. Fuchs (2002). "Immunologic alterations in schizophrenia: neopterin, L-kynurenine, tryptophan and T-cell subsets in the acute stage of illness." PTERIDINES-BERLIN- 13(1): 9-14. 
Stastny, J. r., A. Konstantinidis, M. J. Schwarz, N. E. Rosenthal, O. Vitouch, S. Kasper and A. Neumeister (2003). "Effects of tryptophan depletion and catecholamine depletion on immune parameters in patients with seasonal affective disorder in remission with light therapy." Biological Psychiatry 53(4): 332-337.

Steiner, M., E. Dunn and L. Born (2003). "Hormones and mood: from menarche to menopause and beyond." Journal of Affective Disorders 74(1): 67-83.

Stubner, S., T. Schon, F. Padberg, S. J. Teipel, M. J. Schwarz, A. Haslinger, K. Buch, R. Dukoff, R. Lasser, N. Muller, T. Sunderland, S. I. Rapoport, H. J. Moller and H. Hampel (1999). "Interleukin-6 and the soluble IL-6 receptor are decreased in cerebrospinal fluid of geriatric patients with major depression: no alteration of soluble gp130." Neurosci Lett 259(3): 145-148.

Takahashi, S. (1976). "Reduction of blood platelet serotonin levels in manic and depressed patients." Folia Psychiatr Neurol Jpn 30(4): 475-486.

Tang, B., H. Jia, R. J. Kast and E. A. Thomas (2013). "Epigenetic changes at gene promoters in response to immune activation in utero." Brain Behav Immun 30: 168-175.

Tripp, A., H. Oh, J. P. Guilloux, K. Martinowich, D. A. Lewis and E. Sibille (2012). "Brain-derived neurotrophic factor signaling and subgenual anterior cingulate cortex dysfunction in major depressive disorder." Am J Psychiatry 169(11): 1194-1202.

Van den Hove, D. L. A., H. W. M. Steinbusch, A. Scheepens, W. D. J. Van de Berg, L. A. M. Kooiman, B. J. G. Boosten, J. Prickaerts and C. E. Blanco (2006). "Prenatal stress and neonatal rat brain development." Neuroscience 137(1): 145-155.

Veenstra van Nieuwenhoven, A. L., A. Bouman, H. Moes, M. J. Heineman, L. F. de Leij, J. Santema and M. M. Faas (2002). "Cytokine production in natural killer cells and lymphocytes in pregnant women compared with women in the follicular phase of the ovarian cycle." Fertil Steril 77(5): 1032-1037.

Vigod, S. N. and D. E. Stewart (2009). "Emergent research in the cause of mental illness in women across the lifespan." Current Opinion in Psychiatry 22(4): 396-400.

Vivekanandan, P., H. D. Daniel, R. Kannangai, F. Martinez-Murillo and M. Torbenson (2010). "Hepatitis B virus replication induces methylation of both host and viral DNA." J Virol 84(9): 4321-4329.

Walker, A. J., Y. Kim, J. B. Price, R. P. Kale, J. A. McGillivray, M. Berk and S. J. Tye (2014). "Stress, Inflammation, and Cellular Vulnerability during Early Stages of Affective Disorders: Biomarker Strategies and Opportunities for Prevention and Intervention." Front Psychiatry 5: 34. 
Waly, M. I., M. Hornig, M. Trivedi, N. Hodgson, R. Kini, A. Ohta and R. Deth (2012). "Prenatal and Postnatal Epigenetic Programming: Implications for $\mathrm{Gl}$, Immune, and Neuronal Function in Autism." Autism Research and Treatment 2012: 13.

Wang, H., X.-H. Meng, H. Ning, X.-F. Zhao, Q. Wang, P. Liu, H. Zhang, C. Zhang, G.-H. Chen and D.-X. Xu (2010). "Age- and gender-dependent impairments of neurobehaviors in mice whose mothers were exposed to lipopolysaccharide during pregnancy." Toxicology Letters 192(2): 245-251.

Watanabe, Y., M. Fujiwara, R. Yoshida and O. Hayaishi (1980). "Stereospecificity of hepatic L-tryptophan 2,3-dioxygenase." Biochem J 189(3): 393-405.

Wegmann, T. G., H. Lin, L. Guilbert and T. R. Mosmann (1993). "Bidirectional cytokine interactions in the maternal-fetal relationship: is successful pregnancy a TH2 phenomenon?" Immunol Today 14(7): 353-356.

Weiss, G., C. Murr, H. Zoller, M. Haun, B. Widner, C. Ludescher and D. Fuchs (1999). "Modulation of neopterin formation and tryptophan degradation by Th1and Th2-derived cytokines in human monocytic cells." Clin Exp Immunol 116(3): 435-440.

Weizman, R., N. Laor, E. Podliszewski, I. Notti, M. Djaldetti and H. Bessler (1994). "Cytokine production in major depressed patients before and after clomipramine treatment." Biol Psychiatry 35(1): 42-47.

Willeit, M., N. Praschak-Rieder, A. Neumeister, W. Pirker, S. Asenbaum, O. Vitouch, J. Tauscher, E. Hilger, J. Stastny, T. Brucke and S. Kasper (2000). "[123I]beta-CIT SPECT imaging shows reduced brain serotonin transporter availability in drug-free depressed patients with seasonal affective disorder." Biol Psychiatry 47(6): 482-489.

Winter, C., A. Djodari-Irani, R. Sohr, R. Morgenstern, J. Feldon, G. Juckel and U. Meyer (2009). "Prenatal immune activation leads to multiple changes in basal neurotransmitter levels in the adult brain: implications for brain disorders of neurodevelopmental origin such as schizophrenia." Int J Neuropsychopharmacol 12(4): 513-524.

Wischhof, L., E. Irrsack, C. Osorio and M. Koch (2015). "Prenatal LPS-exposure - a neurodevelopmental rat model of schizophrenia - differentially affects cognitive functions, myelination and parvalbumin expression in male and female offspring." Progress in Neuro-Psychopharmacology and Biological Psychiatry 57(0): 17-30.

Wolff, A. R. and D. K. Bilkey (2008). "Immune activation during mid-gestation disrupts sensorimotor gating in rat offspring." Behav Brain Res 190(1): 156159.

Wu, H. Q., E. F. Pereira, J. P. Bruno, R. Pellicciari, E. X. Albuquerque and R. Schwarcz (2010). "The astrocyte-derived alpha7 nicotinic receptor antagonist 
kynurenic acid controls extracellular glutamate levels in the prefrontal cortex." J Mol Neurosci 40(1-2): 204-210.

Xuan, I. C. Y. and D. R. Hampson (2014). "Gender-Dependent Effects of Maternal Immune Activation on the Behavior of Mouse Offspring." PLoS ONE 9(8): e104433.

Yoshida, T., M. Ishikawa, T. Niitsu, M. Nakazato, H. Watanabe, T. Shiraishi, A. Shiina, T. Hashimoto, N. Kanahara, T. Hasegawa, M. Enohara, A. Kimura, M. lyo and K. Hashimoto (2012). "Decreased Serum Levels of Mature Brain-Derived Neurotrophic Factor (BDNF), but Not Its Precursor proBDNF, in Patients with Major Depressive Disorder." PLoS ONE 7(8): e42676.

Yoshimura, R., M. Mitoma, A. Sugita, H. Hori, T. Okamoto, W. Umene, N. Ueda and J. Nakamura (2007). "Effects of paroxetine or milnacipran on serum brainderived neurotrophic factor in depressed patients." Prog Neuropsychopharmacol Biol Psychiatry 31(5): 1034-1037.

Zavitsanou, K., C. K. Lim, T. Purves-Tyson, T. Karl, M. Kassiou, S. D. Banister, G. J. Guillemin and C. S. Weickert (2014). "Effect of maternal immune activation on the kynurenine pathway in preadolescent rat offspring and on MK801induced hyperlocomotion in adulthood: Amelioration by COX-2 inhibition." Brain, Behavior, and Immunity 41(0): 173-181.

Zdilar, D., K. Franco-Bronson, N. Buchler, J. A. Locala and Z. M. Younossi (2000). "Hepatitis C, interferon alfa, and depression." Hepatology 31(6): 12071211.

Zelante, T., F. Fallarino, F. Bistoni, P. Puccetti and L. Romani (2009). "Indoleamine 2,3-dioxygenase in infection: the paradox of an evasive strategy that benefits the host." Microbes and Infection 11(1): 133-141.

Zhou, H. (2012). "Maternal Infection and Neurodevelopmental Disorders in the Offspring." American Journal of Immunology 8(1): 10.

Zorrilla, E. P., L. Luborsky, J. R. McKay, R. Rosenthal, A. Houldin, A. Tax, R. McCorkle, D. A. Seligman and K. Schmidt (2001). "The relationship of depression and stressors to immunological assays: a meta-analytic review." Brain Behav Immun 15(3): 199-226.

Zuckerman, L., M. Rehavi, R. Nachman and I. Weiner (2003). "Immune activation during pregnancy in rats leads to a postpubertal emergence of disrupted latent inhibition, dopaminergic hyperfunction, and altered limbic morphology in the offspring: a novel neurodevelopmental model of schizophrenia." Neuropsychopharmacology 28(10): 1778-1789.

Zuckerman, L. and I. Weiner (2005). "Maternal immune activation leads to behavioral and pharmacological changes in the adult offspring." $\underline{\text { J Psychiatr }}$ Res 39(3): 311-323. 



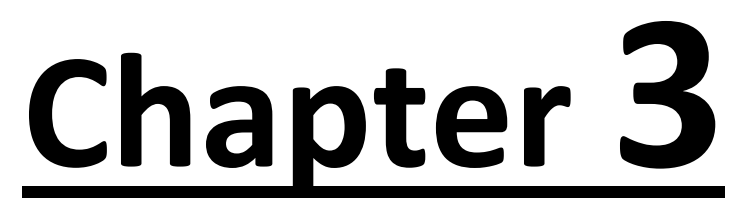

\section{Effects of subcutaneous LPS injection on gestational length and intra-uterine and neonatal mortality in mice}

Yara Pujol Lopez; Harry WM Steinbusch; Bart PF Rutten; Gunter Kenis; Daniel LA van den Hove; Aye M Myint

Neuroimmunomodulation 2015;22(4):274-8 


\section{Abstract}

Infection during pregnancy can predispose offspring to develop various psychiatric disorders such as depression in later life. In order to investigate potential mechanisms underlying these associations, animal models of maternal infection have been employed. As such, lipopolysaccharide (LPS) has been commonly used to mimic a bacterial infection in pregnant mice. The original aim of our study was to investigate the effects of different doses of subcutaneous lipopolysaccharide (LPS) administration on affective behaviour in adult mouse offspring. In the present paper, however, we report that subcutaneous LPS administration has a profound impact upon gestational length, litter size and perinatal mortality in the offspring, even at a relatively low dose.

Pregnant mice were randomly divided into 3 groups, receiving either a high $(2 \mathrm{mg} / \mathrm{kg}$ ) or low $(0.5 \mathrm{mg} / \mathrm{kg})$ dose of LPS or phosphate buffered saline (PBS) by means of subcutaneous injection. Subsequently, the effects on gestational length, litter size and perinatal mortality in the offspring were assessed. After subcutaneous injection with a high dose of LPS, we observed a significant decrease in gestational length and an increase in neonatal mortality. When the low dose administered, a tendency towards reduced litter size was observed, most likely reflecting increased intra-uterine mortality in response to prenatal maternal LPS exposure.

We show that subcutaneous LPS administration of $2 \mathrm{mg} / \mathrm{kg}$ to pregnant mice in the last phase of gestation should be avoided because of high offspring mortality rates, whereas the subcutaneous injection of $0.5 \mathrm{mg} / \mathrm{kg}$ of LPS seems to result in the reabsorption of foetuses. 


\section{Introduction}

Infection during pregnancy has been reported to increase the susceptibility of the offspring to develop psychopathology later in life (Patterson 2002, Meyer, Yee et al. 2007). For instance, increased risks of schizophrenia, bipolar disorder and autism have been reported in offspring of women infected with viruses or bacteria during pregnancy (Brown 2006, Atladottir, Thorsen et al. 2010, Parboosing, Bao et al. 2013). In order to investigate potential mechanisms underlying these associations, animal models of maternal infection have been employed. As such, lipopolysaccharide (LPS) has been commonly used to mimic a bacterial infection in pregnant mice. Through the toll-like receptor 4 (TLR4), LPS increases the production of proinflammatory cytokines, which are known to interfere with the development of the central nervous system (Boksa 2010). In the fetal brain, cytokines are known to regulate the function and development of neurons, being involved in neuroprotective and neurodegenerative processes (Licinio 1997). Different effects of LPS in rodents have been reported, such as modulation of the hypothalamus-pituitary-adrenal (HPA) axis, resulting in increased corticosterone levels (Grinevich, Ma et al. 2001). After LPS exposure during gestation, several behavioural changes have been observed in the offspring in adulthood, e.g. impairments in spatial learning and memory (Golan, Lev et al. 2005, Lante, Meunier et al. 2008, Hao, Hao et al. 2010).

Intraperitoneal (i.p.) injection of LPS to pregnant mothers is known to result in fetal death depending on the dose administered (Renaud, Cotechini et al. 2011). In addition to the dose injected, the time point of LPS injection plays an important role regarding changes in protein levels of cytokines in the 
brains of foetuses (Chlodzinska, Gajerska et al. 2011). Noteworthy, the great majority of prenatal LPS studies have used i.p. injections in pregnant mice. (Buhimschi, Buhimschi et al. 2003, Xu, Chen et al. 2006, Salminen, Paananen et al. 2008, Chlodzinska, Gajerska et al. 2011, Renaud, Cotechini et al. 2011). Considering the fact that i.p. injections have a failure rate in the order of 1020\% (Gaines Das and North 2007), subcutaneous (s.c.) injections may be favored - particularly when dealing with pregnant animals, where misplacement of the injection can cause severe damage to both the mother and foetus. However, little is known about potentially adverse effects of s.c. LPS injections in pregnant animals. Whereas originally, the aim of our study was to investigate the effects of different doses of s.c. LPS administration on affective behaviour in adult offspring, in the present paper, we report that s.c. LPS administration has a profound impact upon gestational length, litter size and perinatal mortality in the offspring, even at a relatively low dose.

\section{Material and methods}

\subsubsection{Animals}

C57BL/6JRccHsd mice were obtained from Harlan Laboratories (Eystrup, Germany). Mice were housed 4 per cage in single-sex groups in individually ventilated cages (IVC) with cage sizes $480 \times 375 \times 210 \mathrm{~mm}$ (depth $\mathrm{x}$ width $\mathrm{x}$ high) under specified pathogen free (SPF) conditions and maintained on a 12/12h light-dark cycle (light on at 12am) and temperature-controlled environment (relativity humidity: $55 \% \pm 5 \%$; temperature: $22^{\circ} \mathrm{C} \pm 2{ }^{\circ} \mathrm{C}$; room air exchange rate: 15$)$. Mice were s.c. 
injected with a transponder for identification purposes. Breeding food (Ssniff, Germany) and water were allowed ad libitum. Cages were changed every week. Experiments were conducted in accordance with permissions from the government and the veterinarian administration of Oberbayern (AZ: 55.2-1-54-2531-61-10). After arriving at the local animal facilities, the mice were allowed to habituate to their new environment for 3 weeks. During this period, mice were chipped to facilitate their identification. For mating, females were housed together with a male mouse for 3 days ( 1 male and 1 female per cage). Afterwards, females were housed individually. Three weeks after delivery, offspring were weaned and separated by sex (4 mice/cage). Offspring were chipped when they were 6 weeks old.

\subsubsection{Mating and induction of prenatal infection}

Approximately 8 weeks after birth, male and female mice were coupled again for 3 days. At GD17, pregnancy was confirmed by visual inspection of the dam in combination with weight gain analysis (increase between day of mating and the day of the injection). Pregnant mice were randomly divided into 3 groups (high dose of LPS [ $n=21$ ], a low dose of LPS [ $n=16$ ] and phosphate buffered saline [PBS; $n=20]$ ). Dams were s.c. injected with 0.5 or $2 \mathrm{mg} / \mathrm{kg}$ LPS (from Salmonella enterica serotype enteritidis, Sigma Aldrich (Germany)) or an equivalent volume of PBS. Following injection, mice were observed daily until delivery. Postnatal mortality was calculated by comparing the number of born pups found dead and the total number of pups counted during the observation period. Of note, 
when assessing postnatal mortality, 4 animals were excluded in the group treated with a low dose of LPS, as these 4 animals were confirmed to be pregnant at GD17, but never delivered.

\section{$\underline{2.2 \text { Statistical analysis }}$}

To define statistically significant differences in gestational length, litter size and mortality, data were first subjected to analysis of variance (ANOVA) with IBM SPSS Statistics 20 (SPSS Inc., USA). Significant overall effects were subsequently examined in more detail using post-hoc Bonferroni tests. The level of significance was set at $p<0.05$ in all cases.

\section{Results}

Overall, significant effects of LPS administration were found for gestational length $\left(F_{2,54}=26.366 ; p<0.001\right)$ and neonatal mortality $\left(F_{2,50}=93.997\right.$; $p<0.001)$, while a trend towards a significant effect on litter size $\left(F_{2,54}=2.700\right.$; $p=0.076$ ) was observed (see Figure 1). Already one day after injection, some dams treated with the high dose of LPS started to deliver. This led to a significant decrease in gestational length in mothers treated with the high dose of LPS when compared to the control group $(p<0.001)$, while no difference in gestational length was observed between dams injected with the low dose of LPS and control dams $(p=1)$. LPS mothers given the high dose showed a reduction in gestational length compared to those injected with a low dose $(p<0.001)$. In addition, litter size tended to be reduced in dams injected with a low dose of LPS when compared to controls, although this difference did not reach statistical significance $(p=0.095)$. Further, no 76 
significant differences were observed between the high dose of LPS and controls $(p=1)$; and between the two doses of LPS $(p=0.211)$. A remarkable effect was observed regarding neonatal mortality. After injection with the high dose of LPS, almost all pups died within a few days after birth (>95\%), whereas mortality in control offspring was almost negligible $(1.5 \%, p<0.001)$. A mortality rate of $16.67 \%$ was detected following administration of the low dose of LPS, but this effect did not reach significance when compared to controls

$(p=0.233)$. When comparing both doses of LPS, a significant increase in mortality was observed in pups from mothers injected with a high dose of LPS $(p<0.001)$. 

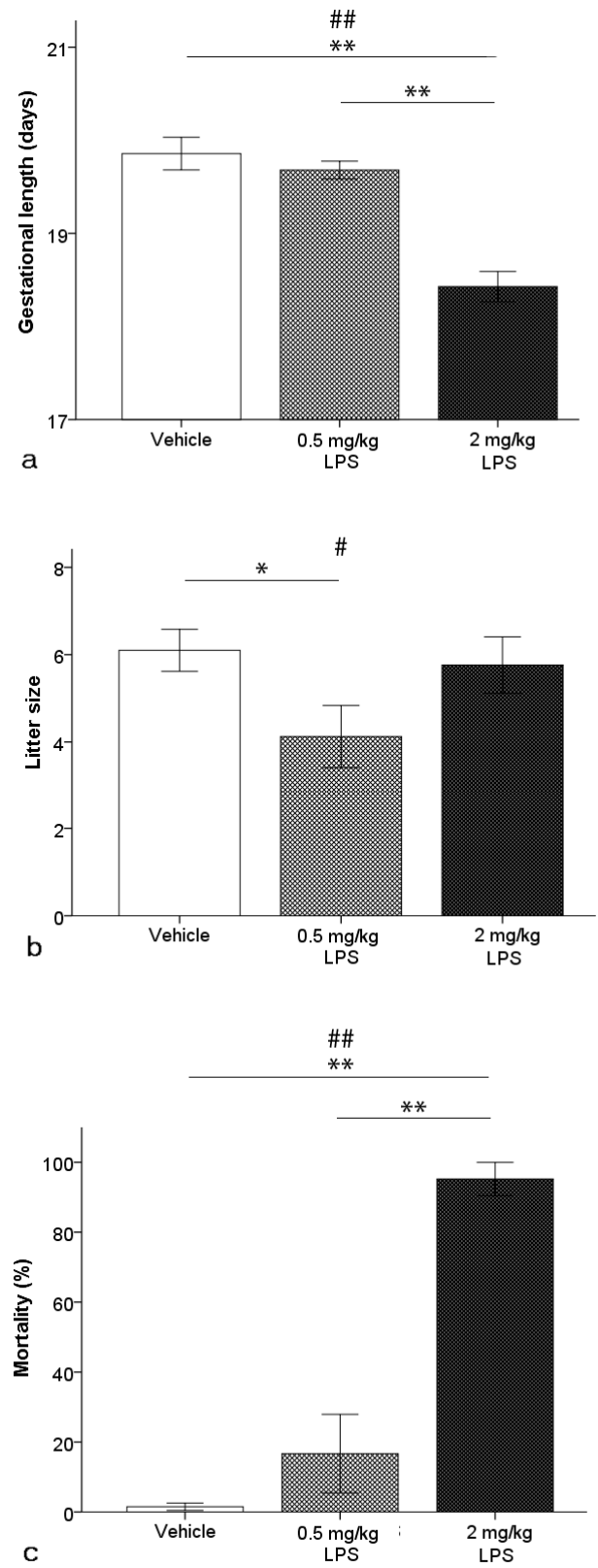

Figure 1. Overall, significant LPS effects were found on gestational length $\left(F_{2,54}=26.366 ; \quad p<0.001\right)$ and mortality $\left(F_{2,50}=93.997 ; p<0.001\right)$, while a trend towards a significant effect on litter size $\left(F_{2,54}=2.700 ; p=0.076\right)$ was observed.

a: More specifically, a reduced gestational length was observed after subcutaneous administration of a high dose of LPS when compared to control $(p<0.001)$. A similar effect was found when analyzing both doses of LPS $(p<0.001)$.

$\mathrm{b}$ : Litter size tended to be reduced after treatment with a low dose of LPS $(p=0.095)$ when compared to controls.

c: after subcutaneous injection with the high dose of LPS, neonatal mortality was increased when compared to both the low dose of LPS $(p<0.001)$ and controls $(p<0.001)$. Bars represent means + S.E. \# $p<0.1, \#$ \# $<0.001$ (ANOVA), * $p<0.1, * *$ $\mathrm{p}<0.001$ (Bonferroni). 


\section{Discussion}

In the present paper, we report that s.c. LPS administration in the mouse has a profound impact upon gestational length, litter size and perinatal mortality in the offspring, even at a relatively low dose. More specifically, our study shows that s.C. administration of LPS at a dose of $2 \mathrm{mg} / \mathrm{kg}$ to pregnant mice resulted in a significant reduction in gestational length and a high neonatal mortality rate, whereas the s.c. injection with $0.5 \mathrm{mg} / \mathrm{kg}$ LPS seemed to be associated with increased prenatal mortality, reflecting reabsorption of foetuses.

\section{$\underline{4.1 \text { Route of administration }}$}

Whereas other publications reported similar effects after prenatal LPS administration, the exact effects seem to largely depend on the dose, route of administration and gestational day of exposure or on the combination of these factors (Mijovic, Zakar et al. 2002, Nakamura, Mori et al. 2003). Most of the studies used i.p. administration of LPS (e.g. (Xu, Chen et al. 2006, Salminen, Paananen et al. 2008)), while little is known about other ways of administration such as intrauterine or intravenous injections (Kohmura, Kirikae et al. 2000, Wang, Hagberg et al. 2007). Importantly, between 10 and $20 \%$ of regular i.p. injections are not placed in the peritoneal cavity, but in the intestine or stomach and this frequency could not be minimized after modifications of the procedure (Steward, Ornellas et al. 1968, Miner, Koehler et al. 1969, Gaines Das and North 2007). In fact, in case of injecting pregnant dams, such adverse effects may be expected to be even worse, including direct harm to the foetus. 
In addition, other disadvantages such as splenic trauma, serosal hemorrhage and contamination are reported (Cho, Lee et al. 2011). Hence, s.c. injections may represent more a more suitable route of administration when dealing with pregnant mice.

\subsection{Dose dependent effect on prenatal and neonatal mortality}

As mentioned above, the dose of LPS and the timing of the injection are considered to be critical factors that may impact upon fetal death. However, to date, no report has indicated which dose of s.c. LPS is sufficient to trigger a response in the mother and foetus without causing death. A limited number of articles have been published studying the effects of zinc supplementation and teratogenicity after prenatal exposure to LPS (Carey, Berbee et al. 2003, Chua, Rofe et al. 2006, Chua, Cowley et al. 2012). However, no evidence was reported on effects on intra-uterine or neonatal mortality, litter size or gestational length. In the present study, litter size tended to be reduced when using a low dose of LPS. In fact, 4 females within this group were confirmed to be pregnant at the time of LPS injection (GD17), but never delivered, pointing at reabsorption of foetuses. Interestingly, it has been reported that prenatal maternal i.p. administration of high doses of LPS causes fetal resorption, whereas exposure to low doses resulted in normal delivery (Chlodzinska, Gajerska et al. 2011). Contra-intuitively, we only observed a reduction in litter size after prenatal administration of a low dose, but not after using a high dose of LPS. This lack of effect when using the high dose could be explained by the fact that the high dose of LPS induced an abrupt 
premature delivery, concomitant with a massive increase in neonatal instead of prenatal - mortality, allowing no opportunity for intrauterine absorption. Although it was expected that some pups would die after exposure to LPS, nonetheless, the high rate of neonatal mortality (>95\%) observed after administration of a high dose of LPS compared to the low dose $(16.67 \%)$ was remarkable.

\section{$\underline{4.3 \text { Concluding remarks }}$}

Evidently, it would be useful, to find out more about the exact effects of prenatal LPS administration in pups when using different doses, routes and time points of administration in order to choose the suitable exposure for future experiments. Although most problems can be expected when using i.p. injections, this route of administration is still mostly employed in mouse models. Therefore, we investigated the effects of different doses of LPS using s.c. injections. In our study, using s.c. LPS injections, we could avoid the problem of misplacing the agent or directly harming the foetus when injecting the pregnant dams. Altogether, we conclude that s.c. LPS administration in the mouse has a profound impact upon gestational length, litter size and perinatal mortality in the offspring, even at a relatively low dose. Future work should elucidate to which extent lower doses of LPS induce short- and/or long-term behavioural changes in the offspring and which exact molecular mechanisms are involved. 


\section{Acknowledgements}

The study was supported by the Commission of European Communities $7^{\text {th }}$ Framework Programme collaborative project "MOODINFLAME" (Grant No. 22963).

The authors would like to thank Andreas Weiss, Andrea Millet and Prof. Markus Schwarz for their guidance and help in the performance of the experiments. They were employed in Ludwig-Maximilian University, Munich, with financial compensation. We also thank Jo Stevens for the statistical advice. 


\section{References}

Atladottir, H. O., P. Thorsen, L. Ostergaard, D. E. Schendel, S. Lemcke, M. Abdallah and E. T. Parner (2010). "Maternal infection requiring hospitalization during pregnancy and autism spectrum disorders." J Autism Dev Disord 40(12): 1423-1430.

Boksa, P. (2010). "Effects of prenatal infection on brain development and behavior: A review of findings from animal models." Brain, Behavior, and Immunity 24(6): 881-897.

Brown, A. S. (2006). "Prenatal infection as a risk factor for schizophrenia." Schizophrenia bulletin 32(2): 200-202.

Buhimschi, I. A., C. S. Buhimschi and C. P. Weiner (2003). "Protective effect of Nacetylcysteine against fetal death and preterm labor induced by maternal inflammation." American Journal of Obstetrics and Gynecology 188(1): 203-208.

Carey, L. C., P. L. Berbee, P. Coyle, J. C. Philcox and A. M. Rofe (2003). "Zinc treatment prevents lipopolysaccharide-induced teratogenicity in mice." Birth Defects Res A Clin Mol Teratol 67(4): 240-245.

Chlodzinska, N., M. Gajerska, K. Bartkowska, K. Turlejski and R. L. Djavadian (2011). "Lipopolysaccharide injected to pregnant mice affects behavior of their offspring in adulthood." Acta Neurobiol Exp (Wars) 71(4): 519-527.

Cho, Y. J., Y. A. Lee, J. W. Lee, J. I. Kim and J. S. Han (2011). "Kinetics of proinflammatory cytokines after intraperitoneal injection of tribromoethanol and a tribromoethanol/xylazine combination in ICR mice." Laboratory animal research 27(3): 197-203.

Chua, J. S., C. J. Cowley, J. Manavis, A. M. Rofe and P. Coyle (2012). "Prenatal exposure to lipopolysaccharide results in neurodevelopmental damage that is ameliorated by zinc in mice." Brain Behav Immun 26(2): 326-336.

Chua, J. S., A. M. Rofe and P. Coyle (2006). "Dietary zinc supplementation ameliorates LPS-induced teratogenicity in mice." Pediatr Res 59(3): 355358.

Gaines Das, R. and D. North (2007). "Implications of experimental technique for analysis and interpretation of data from animal experiments: outliers and increased variability resulting from failure of intraperitoneal injection procedures." Lab Anim 41(3): 312-320.

Golan, H. M., V. Lev, M. Hallak, Y. Sorokin and M. Huleihel (2005). "Specific neurodevelopmental damage in mice offspring following maternal inflammation during pregnancy." Neuropharmacology 48(6): 903-917.

Grinevich, V., X. M. Ma, J. P. Herman, D. Jezova, I. Akmayev and G. Aguilera (2001). "Effect of repeated lipopolysaccharide administration on tissue cytokine 
expression and hypothalamic-pituitary-adrenal axis activity in rats." Neuroendocrinol 13(8): 711-723.

Hao, L. Y., X. Q. Hao, S. H. Li and X. H. Li (2010). "Prenatal exposure to lipopolysaccharide results in cognitive deficits in age-increasing offspring rats." Neuroscience 166(3): 763-770.

Kohmura, Y., T. Kirikae, F. Kirikae, M. Nakano and I. Sato (2000). "Lipopolysaccharide (LPS)-induced intra-uterine fetal death (IUFD) in mice is principally due to maternal cause but not fetal sensitivity to LPS." Microbiology and immunology 44(11): 897-904.

Lante, F., J. Meunier, J. Guiramand, M. C. De Jesus Ferreira, G. Cambonie, R. Aimar, C. Cohen-Solal, T. Maurice, M. Vignes and G. Barbanel (2008). "Late Nacetylcysteine treatment prevents the deficits induced in the offspring of dams exposed to an immune stress during gestation." Hippocampus 18(6): 602-609.

Licinio, J. (1997). "Central nervous system cytokines and their relevance for neurotoxicity and apoptosis." J Neural Transm Suppl 49: 169-175.

Meyer, U., B. K. Yee and J. Feldon (2007). "The neurodevelopmental impact of prenatal infections at different times of pregnancy: the earlier the worse?" The Neuroscientist : a review journal bringing neurobiology, neurology and psychiatry 13(3): 241-256.

Mijovic, J. E., T. Zakar, D. B. Zaragoza and D. M. Olson (2002). Tyrphostins inhibit lipopolysaccharide induced preterm labor in mice. Journal of Perinatal Medicine. 30: 297.

Miner, N. A., J. Koehler and L. Greenaway (1969). "Intraperitoneal injection of mice." Applied microbiology 17(2): 250-251.

Nakamura, A., Y. Mori, K. Hagiwara, T. Suzuki, T. Sakakibara, T. Kikuchi, T. Igarashi, M. Ebina, T. Abe, J. Miyazaki, T. Takai and T. Nukiwa (2003). "Increased susceptibility to LPS-induced endotoxin shock in secretory leukoprotease inhibitor (SLPI)-deficient mice." J Exp Med 197(5): 669-674.

Parboosing, R., Y. Bao, L. Shen, C. A. Schaefer and A. S. Brown (2013). "Gestational Influenza and Bipolar Disorder in Adult OffspringGestational Influenza and Bipolar Disorder." JAMA Psychiatry: 1-8.

Patterson, P. H. (2002). "Maternal infection: window on neuroimmune interactions in fetal brain development and mental illness." Current Opinion in Neurobiology 12(1): 115-118.

Renaud, S. J., T. Cotechini, J. S. Quirt, S. K. Macdonald-Goodfellow, M. Othman and C. H. Graham (2011). "Spontaneous pregnancy loss mediated by abnormal maternal inflammation in rats is linked to deficient uteroplacental perfusion." J Immunol 186(3): 1799-1808. 
Salminen, A., R. Paananen, R. Vuolteenaho, J. Metsola, M. Ojaniemi, H. AutioHarmainen and M. Hallman (2008). "Maternal endotoxin-induced preterm birth in mice: fetal responses in toll-like receptors, collectins, and cytokines." Pediatr Res 63(3): 280-286.

Steward, J. P., E. Ornellas, K. Beernink and W. H. Northway (1968). "Errors in the technique of intraperitoneal injection of mice." Applied microbiology 16(9): 1418.

Wang, X., H. Hagberg, C. Zhu, B. Jacobsson and C. Mallard (2007). "Effects of intrauterine inflammation on the developing mouse brain." Brain Res 1144: 180-185.

Xu, D. X., Y. H. Chen, H. Wang, L. Zhao, J. P. Wang and W. Wei (2006). "Tumor necrosis factor alpha partially contributes to lipopolysaccharide-induced intra-uterine fetal growth restriction and skeletal development retardation in mice." Toxicol Lett 163(1): 20-29. 



\section{Chapter 4}

\section{Quinolinic acid-immunoreactivity in the naïve mouse brain}

Yara Pujol Lopez; Gunter Kenis; Bart PF Rutten; Aye M Myint; Harry WM Steinbusch; Daniel LA van den Hove 


\section{$\underline{\text { Abstract }}$}

Quinolinic acid (QUIN) has been suggested to be involved in infections, inflammatory neurological disorders and in the development of psychiatric disorders. In this view, several studies have been performed to investigate QUIN localization in the brain and its neurotoxic effects. However, evidence is lacking regarding QUIN in healthy, control conditions. The aim of this study was to investigate the region-specific distribution and pattern of QUIN expression in the naïve mouse brain. In addition, possible sex differences in QUIN-immunoreactivity and its link with affect-related behavioural observations were assessed.

For this purpose, naïve mice were subjected to the forced swim test (FST) and 20 minutes open field (OF) testing to measure affect-related behaviour. Afterwards, brains were assessed for QUIN-immunoreactivity. QUINimmunoreactivity was particularly observed in the cingulate cortex (CC), highlighting clearly delineated cells, and the thalamic reticular nucleus (TRN), showing a more diffuse staining pattern. Subsequently, QUIN-positive cells in the CC were counted, while QUIN-immunoreactivity in the TRN was examined using gray value measurements. No significant differences between sexes were observed for the number of QUIN-positive cells in the CC, neither in levels of QUIN-immunoreactivity in the TRN. A direct correlation was found between QUIN-positive cells in the CC and QUINimmunoreactivity in the TRN. Moreover, in male mice, a very strong correlation $\left(r_{\mathrm{sp}}=0.943 ; p<0.01\right)$ between QUIN-immunoreactivity at the level of the TRN and motor activity in the OF was observed. Thus, our results 
suggest that QUIN - detected in the CC and the TRN- may play an important role in regulating motor activity in normal conditions. 


\section{Introduction}

Quinolinic acid (QUIN) is an endogenous N-methyl-D-aspartate (NMDA) glutamate receptor agonist produced by the kynurenine pathway, particularly in the liver and the brain. The kynurenine pathway, which has been suggested to be implicated in the development of psychiatric disorders (Asp, Johansson et al. 2011, Myint 2012), involves the catabolism of tryptophan to kynurenine, which in turn can be further metabolised into the intermediates kynurenic acid (KYNA) or QUIN. Alterations in tryptophan, kynurenine, KYNA and QUIN have been reported in patients suffering from depression, schizophrenia and Alzheimer's disease, among others (Nilsson, Linderholm et al. , Németh, Toldi et al. 2006, Myint, Kim et al. 2007, Gulaj, Pawlak et al. 2010). In depressive patients, increased cerebrospinal fluid (CSF) levels of QUIN have been observed (Raison, Dantzer et al. 2010), whereas another recent study showed decreased QUIN-immunoreactivity in the hippocampus of patients suffering from depression (Busse, Busse et al. 2014).

Evidence suggests that the production and accumulation of QUIN in the central nervous system (CNS) is implicated in several inflammatory neurological disorders and infections (Dang, Dale et al. 2000). It is wellknown that during inflammation, cytokines are produced which mediate a subset of distinct inflammatory reactions (Kronfol and Remick 2000, Zhang and An 2007). Already in 1992, a study reported increased QUIN in lumbar CSF and post-mortem brain tissue of patients with inflammatory diseases, but not in CSF of patients with non-inflammatory neurological disease (Heyes, Saito et al. 1992). Moreover, in depression associated with a pro90 
inflammatory status, QUIN-immunoreactivity was found to be increased in the anterior midcingulate cortex ( $\mathrm{aMCC}$ ), the subgenual anterior cingulate cortex (sACC) and the cortical grey matter (Steiner, Walter et al. 2011). QUIN has been reported to aggrevate the negative consequences of proinflammatory cytokines (Maes 2008) and to exert various neurotoxic effects, inducing neuronal cell loss and convulsions (Nemeth, Toldi et al. 2005). QUIN has also been linked to the induction of hippocampal cell death, selective necrosis of granule cells, destruction of postsynaptic elements and a reduction in cerebral cholinergic circuits (Maes, Yirmyia et al. 2009).

In the CNS, QUIN is produced by activated microglia and macrophages. Different studies with discordant results have been conducted using several animal models and human tissues to determine the cellular localisation of QUIN in the brain. In brain tissue from naïve control rats, no QUINimmunoreactivity was observed (Moffett, Espey et al. 1993). However, different types of QUIN-positive cells, such as microglia, macrophage and ameboid-like cells were seen in the subiculum, dorsal hippocampus (CA1) and in the stratum pyramidale of the whole CA1 area in a gerbil model of ischemia (Baratte, Molinari et al. 1998). In another study, high QUINimmunoreactivity was observed only after focal injury of the neocortex in adult rats and was limited to those microglia directly within the lesion tract. In addition, QUIN-immunoreactivity was also seen in reactive cultured microglia and also in microglia of primary cultures of human mixed brain cells after IFN- $\gamma$ stimulation (Guillemin, Smythe et al. 2005, Chung, Leung et al. 2009). 
Despite the fact that research within this field has rapidly expanded in the context of pathological conditions, the normal, physiological role of QUIN is less clear. To date, no report showed the localization of QUIN and the pattern of its staining in the brain of naïve mice. Therefore, we analyzed QUIN-immunoreactivity in the naïve mouse brain. Our first objective was to examine the distribution and localization of QUIN-immunoreactivity. Second, we evaluated whether QUIN-immunoreactivity differs between sexes. Third and last, we investigated the possible association between QUIN-immunoreactivity and a subset of affect-related behavioural phenotypes.

\section{Material and methods}

\section{$\underline{2.1 \text { Animals }}$}

C57BL/6JRccHsd mice were obtained from Harlan Laboratories (Eystrup, Germany). Mice were housed in single-sex groups of 4 mice per cage in individually ventilated cages (IVC $480 \times 375$ x 210 mm [depth $x$ width $x$ high]). Mice were kept under specific pathogen-free (SPF) conditions and maintained on a 12/12h light-dark cycle (light on at 12am) and temperature-controlled environment (relative humidity $55 \% \pm 5 \%$; temperature: $22^{\circ} \mathrm{C} \pm 2^{\circ} \mathrm{C}$, room air exchange rate 15$)$. Cages were changed every week. The habituation period lasted 3 weeks once the mice arrived to the local animal facilities. During this period, mice were subcutaneously injected with a transponder to facilitate identification. Breeding food (Ssniff, Germany) and water were allowed ad libitum. Experiments were performed according to the permissions from the 
government and the veterinarian administration of Oberbayern (AZ: 55.21-54-2531-61-10).

\subsection{Behavioural tests}

\section{$\underline{\text { 2.2.1 Forced swim test }}$}

At 12 weeks of age, mice were tested in the forced swim test (FST). This test consists of 4 vertical glass cylinders (height $57,5 \mathrm{~cm} \times$ diameter 20) filled to a depth of $50 \mathrm{~cm}$ with tap water at $22^{\circ} \mathrm{C}$. Cylinders were situated in front of a frosted glass screen and were illuminated by 4 infrared floodlights (Videor, Germany) behind the screen. Cylinders were separated by opaque partitions so that mice could not see each other. One animal was placed in each cylinder and tested for 6 minutes, between 12 p.m and 5 p.m. After each test, water was changed and mice were dried and put back into their home cages. A wall-mounted camera (type EQ-150, EverFocus, Germany) connected to a converter (ADVC-55 Advanced DV Converter, canopus $\mathrm{GmbH}$, Germany) was used to record the test. Biobserve Forced Swim Test Version 1.5 software (BIObserve, Germany) was employed to analyse the following behaviours: 1) floating (absence of any movement or minor movements needed to keep the head above the water), 2) swimming (movements of the limbs associated with swimming and less severe than those associated with climbing) and 3) struggling (large movements associated with attempts to escape the swim tank). 


\subsubsection{Open Field Test}

One week after the FST, mice were tested in the open field test (OF), consisting of a white PVC box $(50 \times 50 \times 50 \mathrm{~cm}$; Kümpel Kunststoffverarbeitungswerke $\mathrm{GmbH}$, Germany). Two infrared floodlights (type WFL-II/LED30, Videor, Germany), mounted on tripods (type 3150, Cullmann, Germany) were situated on two sides of the OF area (containing 4 boxes). One animal was placed in each box and was allowed to explore the arena for 20 minutes. Testing was performed between 12 p.m and 5 p.m. Boxes were cleaned after each measurement with water and alcohol and mice were returned to their cages. Behaviour was recorded with a camera (type AU-CB600, VIDO, Austria) connected to a converter (ADVC-55 Advanced DV Converter, canopus $\mathrm{GmbH}$, Germany). The video-tracking software Viewer Version 3 (BIObserve, Germany) was used to score the time spent in each zone (s), track length (cm) and zone crossings (counts) in the whole box. Each box was divided as follows: the centre $(34,6 \times 34,6 \mathrm{~cm}$; $W \times D), 4$ walls (each: $7 \times 34,6 \mathrm{~cm}$; W $\times$ D) and 4 corners (each: $7 \times 7 \mathrm{~cm}$; W $\times$ D).

\section{$\underline{2.3 \text { Perfusion and extraction of organs }}$}

Thirteen week old mice were perfused 1 month after OF testing. Male $[n=6]$ and female [n=7] mice were anesthetized with a single intraperitoneal injection of ketamin (Pfizer, Germany) and xylacin (Bayer, Germany). Subsequently, they were perfused with a heparinized saline solution followed by $4 \%$ paraformaldehyde (PFA) and $15 \%$ saturated picric acid. Brains were dissected and postfixed in 4\% PFA overnight 
before being transferred to PBS-containing sodium azide (1\%) and stored at $4^{\circ} \mathrm{C}$.

\subsection{Immunohistochemistry}

Thirty $\mu \mathrm{m}$ coronal brain sections of the brains were cut using a vibratome (Leica VT1200 S, Germany). Free-floating slices were rinsed for 3 times (Tris-buffered Saline wit 0.3\% Triton X-100 (TBST) / Tris-buffered Saline (TBS) / TBST; each for $10 \mathrm{~min}$ ) and pre-incubated with $1 \% \mathrm{H}_{2} \mathrm{O}_{2}$ in TBS to block endogenous peroxidase activity for 30 minutes at room temperature (RT). Tissue sections were washed again as mentioned above and incubated overnight at $4^{\circ} \mathrm{C}$ with the primary polyclonal rabbit anti-QUIN antibody (1:1500 inTBS-T, apDia, Turnout, Belgium) (Gos, Myint et al. 2014). Next, sections were washed and incubated with biotinylated donkey anti-rabbit (1:400 in TBST-T, Jackson Laboratories, US) for 90 minutes at RT. Sections were washed again and tissue was further incubated with avidin-biotin complex (ABC) (1:400 in TBS-T, Vector Immunolabs) for 90 minutes at RT. After incubation, slices were washed twice with TBS for 10 minutes and once with $0.1 \mathrm{M}$ acetate buffer (pH 6.0) for 15 minutes. Subsequently, tissue was incubated for 20 minutes at RT with glucose oxidase and $\mathrm{DAB}$, and washed twice for 15 minutes with $0.1 \mathrm{M}$ acetate buffer ( $\mathrm{pH}$ 6.0). Finally, brain sections were mounted on coated microscope slides, dehydrated using an ethanol series, dried overnight, and coverslipped using Pertex ${ }^{\circledR}$ (HistolabProducts ab, Göteborg, Sweden). 


\section{$\underline{2.5 \text { Microscopy }}$}

Brain sections of 13 animals ( 6 male, 7 female) were analyzed. For cell quantification in the cingulate cortex (CC) we applied the Eclipse E400 microscope from Nikon, while for gray value analysis in the thalamic reticular nucleus (TRN) we used an AX70 microscope (Olympus, analySIS Imaging System) coupled to a digital camera (f-view, Olympus). QUINimmunoreactivity in the TRN was detected from bregma level $-0.82 \mathrm{~mm}$ until bregma level $-1.58 \mathrm{~mm}$ (3-4 sections). These sections were photographed at a 10x and 40x magnification and gray values were analyzed with the ImageJ software. In addition, background correction was performed by subtracting the background signal from the gray values reflecting immunoreactivity. For quantitative studies QUINimmunoreactive cells in the CC were counted at 20x magnification. The counting was performed from bregma level $0.50 \mathrm{~mm}$, where QUINimmunoreactive cells appeared, through all the sections until bregma level $-1.94 \mathrm{~mm}$ (9 sections).

\subsection{Statistical analysis}

The number of QUIN-positive cells and the levels of QUINimmunoreactivity and behavioural measurements were first subjected to non-parametric Kruskal-Wallis tests with IBM SPSS Statistics version 20 (SPSS Inc., USA). In addition, possible correlations between the number of QUIN-positive cells within the CC and the levels of QUINimmunoreactivity in the TRN as well their correlation to behavioural measures in the FST and OF were analyzed using Spearman correlation 
analysis. The level of significance was set at $p<0.05$ in all cases. Graphs were designed with GraphPad Prism 5.

\section{Results}

\subsection{Localization of QUIN-immunoreactivity}

Low levels of QUIN-immunoreactivity were seen throughout the brain, whereas high levels of QUIN-immunoreactivity were observed specifically in two brain regions, i.e. the area ranging from the $\mathrm{CC}$ to the retrosplenial cortex (RSC), as well as in the TRN. To note, as the RSC is considered equivalent to the posterior CC in rodents (Vogt, Vogt et al. 2004, Vann, Aggleton et al. 2009), we will use the term CC to cover cells observed in both the CC and the RSC. QUIN-immunoreactivity in the CC clearly delineated individual cells (see Figure 1). On the other hand, QUINimmunoreactivity in the TRN was much more diffuse, resembling a fibrillary staining pattern (see Figure 2). 


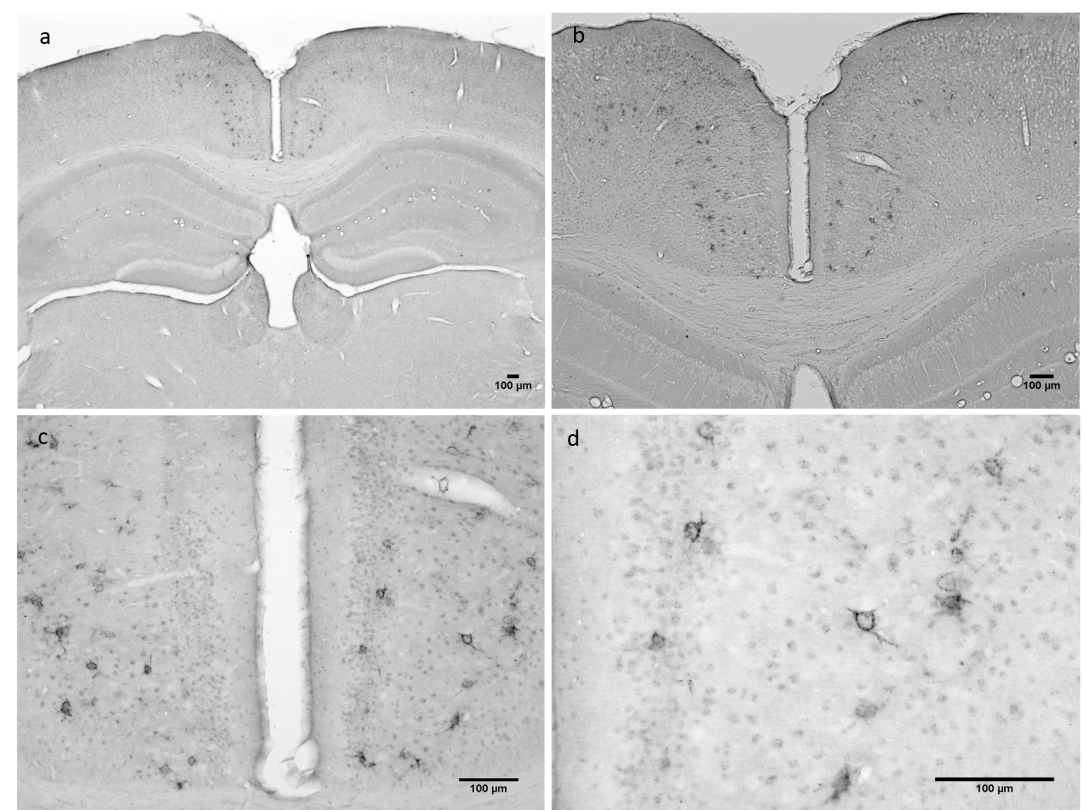

Figure 1. QUIN-positive cells in the cingulate cortex. A, B) Low magnification overview of the brain showing QUIN-positive cells $(2 x, 4 x) . C, D)$ High magnification views of the CC $(10 x, 20 x)$.

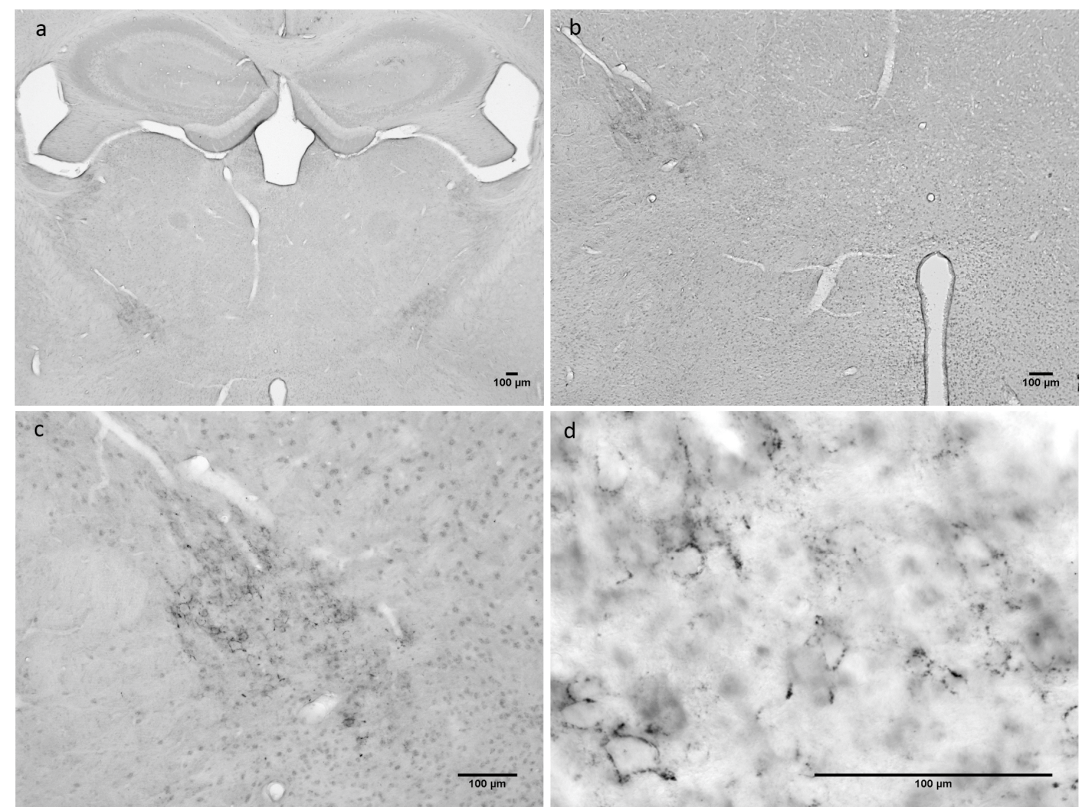

Figure2. QUIN-immunoreactivity in the thalamic reticular nucleus. A, B) Low magnification overview of the brain showing QUIN-immunoreactivity in the TRN $(2 x, 4 x) . C, D)$ High magnification views of the TRN $(10 x, 40 x)$. 


\subsection{Behavioral observations}

Sex differences were found in the distance moved and number of zone crossings both during the complete 20 minutes and first 5 minutes exposure to the $\mathrm{OF}$, indicative of increase activity in females compared to males (all $p<0.05$ ).

3.3 QUIN-immunoreactivity in the $\mathrm{CC}$ and TRN; correlations to behavioural variation

Statistical analysis revealed no significant difference between sexes in the number of QUIN- positive cells within the CC ( $p>0.05$, data not shown). Regarding the TRN, no significant sex effect was found in levels of QUINimmunoreactivity $(p>0.05$, data not shown). Spearman correlation analysis showed a statistically significant positive correlation between the number of QUIN-positive cells in the CC and QUIN-immunoreactivity in the TRN, $r_{s p}=0.569$ (see Table 1 and Figure 3). In addition, in males, we found a highly significant and strong positive correlation between both track length $\left(r_{\mathrm{sp}}=0.943\right)$ and zone crossings $\left(r_{\mathrm{sp}}=0.886\right)$ during 20 minutes'

OF exposure and QUIN-immunoreactivity in the TRN (see Table 1 and Figure 3). 
ב

ํㅓㅇ

$\stackrel{4}{ \pm}$

몽 웅

u

$\stackrel{*}{*}$

ㄷํㅇ

을

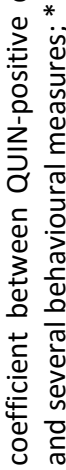

을

ㅎํㅇ

c

है $\frac{\overline{0}}{\frac{0}{2}}$

ํํㄴ

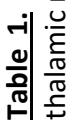

\begin{tabular}{|c|c|c|c|c|c|c|c|}
\hline \multirow{3}{*}{ む } & 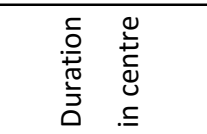 & $\underset{i}{\stackrel{N}{N}}$ & 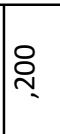 & 咅 & 佫 & ठ્. & 惫 \\
\hline & 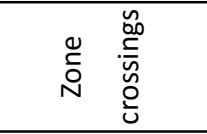 & 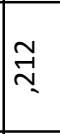 & $\underset{m}{\stackrel{D}{m}}$ & $\stackrel{m}{\text { }}$ & $\stackrel{\infty}{\stackrel{f}{f}}$ & $\begin{array}{l}* \\
\stackrel{0}{\infty} \\
\infty \\
\infty\end{array}$ & ্ָ \\
\hline & 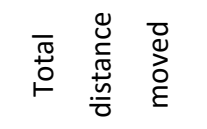 & 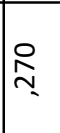 & ‡్丶 & ₹్ & 导 & 苂 & 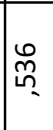 \\
\hline \multirow{3}{*}{ | } & 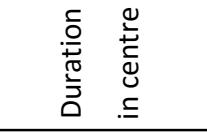 & 访 & 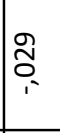 & ㅎ․ & $\vec{\sigma}$ & 氖. & 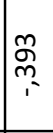 \\
\hline & 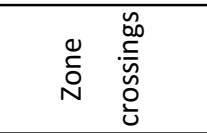 & 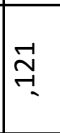 & $\underset{⿱}{\stackrel{m}{F}}$ & $\underset{\text { I্ }}{\stackrel{\Delta}{*}}$ & $\stackrel{\infty}{\infty}$ & 조. & 离. \\
\hline & 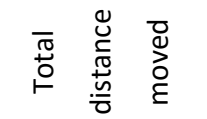 & 令. & $\underset{⿱}{\stackrel{m}{*}}$ & $\underset{f}{\stackrel{m}{f}}$ & 导 & 상. & 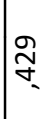 \\
\hline \multirow{3}{*}{ 馬 } & 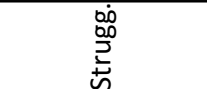 & $\mathscr{J}_{\forall}^{m}$ & 这 & 离 & $\tilde{\infty}$ & 吝 & స్. \\
\hline & $\dot{\xi}$ & 总 & $\underset{7}{\stackrel{7}{*}}$ & $\begin{array}{l}9 \\
6 \\
6\end{array}$ & 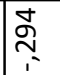 & 免 & po \\
\hline & $\begin{array}{l}\frac{\mathrm{o}}{\mathrm{O}} \\
\frac{1}{4}\end{array}$ & 竎 & \begin{tabular}{|l}
$\infty$ \\
0 \\
0 \\
1
\end{tabular} & $\begin{array}{l}9 \\
\hat{6}\end{array}$ & $\underset{\sim}{\stackrel{\sim}{\sim}}$ & $\begin{array}{l}\overrightarrow{\text { n. }} \\
\text { n. }\end{array}$ & $\begin{array}{l}\text { m. } \\
\text { p. }\end{array}$ \\
\hline \multirow{4}{*}{5} & 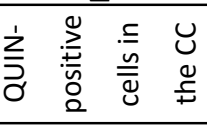 & $\times$ & $\times$ & $x$ & $\begin{array}{l}* \\
\text { * } \\
\text { O̊. } \\
\text { ஸุ. }\end{array}$ & 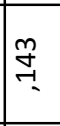 & 站 \\
\hline & 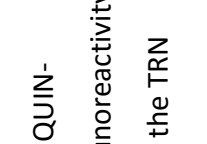 & 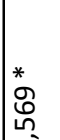 & $\stackrel{\mathscr{m}}{\stackrel{f}{F}}$ & 勇. & $\times$ & $\times$ & $x$ \\
\hline & & \begin{tabular}{|l}
$\frac{\mathscr{y}}{\varepsilon}$ \\
$\overline{\bar{\alpha}}$
\end{tabular} & $\frac{\mathscr{y}}{\sum^{\pi}}$ & 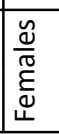 & 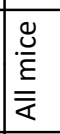 & $\frac{\tilde{\omega}}{\tilde{\pi}}$ & 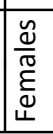 \\
\hline & & & 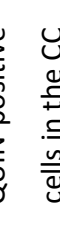 & & $\mid \begin{array}{l}2 \\
\overline{0}\end{array}$ & 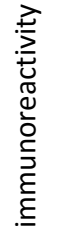 & 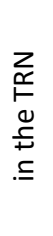 \\
\hline
\end{tabular}



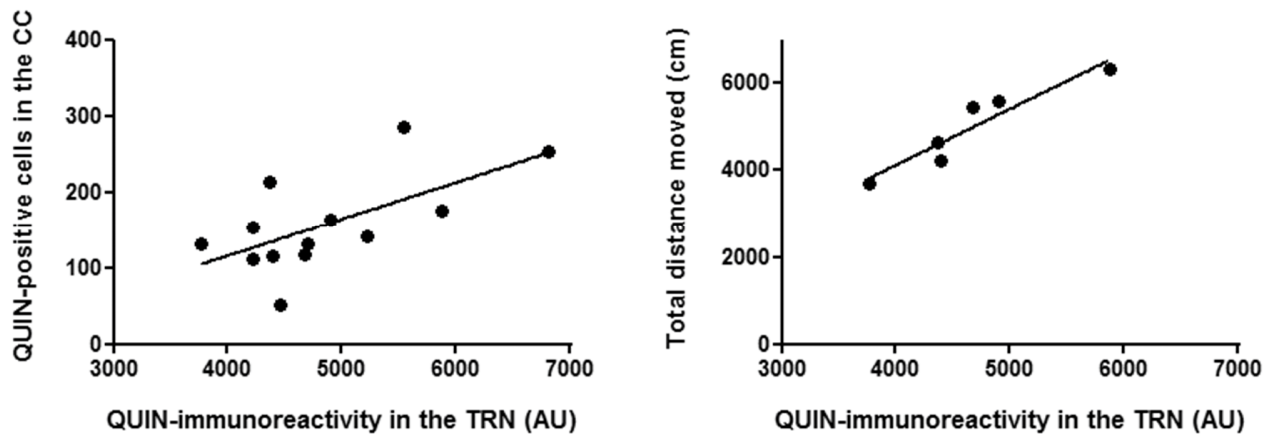

Figure 3. Left: positive correlation between QUIN-immunoreactivity in the TRN and QUIN-positive cells in the $C C, r_{s p}=0.569, p<0.05$, male $n=6$, female $n=7$. Right: positive correlation between track length in OF 20 minutes and QUIN-immunoreactivity in the TRN $r_{s p}=0.943, p<0.01$, male $n=6$.

\section{Discussion}

In the present paper, we show high levels of QUIN-immunoreactivity specifically in the CC and TRN of naïve mice. In the CC, QUINimmunoreactivity delineated individual cells, whereas in the TRN the pattern of staining was more diffuse, resembling a fibrillary staining pattern. Interestingly, in males only, QUIN-immunoreactivity in the TRN showed a highly significant and very strong correlation with the measures of distance moved and zone crossings in the OF. Moreover, the number of QUINpositive cells in the $\mathrm{CC}$ was positively correlated with QUINimmunoreactivity in the TRN.

\subsection{QUIN-positive cells in the CC}

Specifically, QUIN-positive cells were observed in the CC and the RSC. Individual cells were observed with immunoreactivity specifically marking the cell membrane. The CC forms part of the limbic system, which is 
associated with emotion processing, learning and memory (Hadland, Rushworth et al. 2003, Dalley, Cardinal et al. 2004, Kozlovskiy, Vartanov et al. 2012). The RSC is located behind the splenium of the corpus callosum and it is involved in episodic memory, navigation, imagination of future events and processing scenes (Vann, Aggleton et al. 2009). In humans, the RSC forms part of the posterior CC, together with Brodmann areas 23 and 31 (Maddock 1999, Vann, Aggleton et al. 2009). However, in rodents, the posterior CC is equivalent to only the RSC (Vogt, Vogt et al. 2004, Vann, Aggleton et al. 2009). It is reported that the RSC receives input from several areas of the CC (Maddock 1999, Vogt, Vogt et al. 2004). In addition, together with the posterior CC, the RSC has also been involved in emotion processing and memory (Maddock 1999, Vogt, Vogt et al. 2004). Thus, suggesting functions in common for both CC and RSC. The exact role of QUIN in these brain regions remains unclear. Interestingly, several studies reported impaired memory (Beninger, Jhamandas et al. 1986, Misztal, Skangiel-Kramska et al. 1996) and altered spatial reference memory (Shear, Dong et al. 1998) in rodents after injection of QUIN in the brain.

\subsection{QUIN-immunoreactivity in the TRN}

QUIN-immunoreactivity was observed in the TRN and partially disseminated to the zona incerta (ZI) in the hypothalamus. Here, we observed a slightly different, more diffuse staining pattern. The TRN is part of the ventral thalamus that contains gamma amino butyric acid (GABA)ergic cells. It is involved in the regulation of the sensory, 
motor and limbic system as well as in thalamocortical communication (Pinault 2004, Lam and Sherman 2011). The $\mathrm{Zl}$ is a nucleus located ventral to the thalamus and contiguous with the TRN. Its exact function is still unknown, but it is suggested to be involved in the transmission of brainstem viscerosensory and somatosensory information (Power and Mitrofanis 2001). Interestingly, QUIN-immunoreactivity in males was strongly correlated to locomotion during 20 minutes' OF exposure, whereas we did not observe any correlation between QUINimmunoreactivity and behavioural measures during the first 5 minutes of exposure to the OF. Thus, these data suggest a modulatory role for QUIN at the level of the TRN in mediating locomotion and possibly even hyperactivity, without affecting anxiety-related behaviour. Interestingly, the TRN has been implicated in attention-deficit hyperactivity disorder (ADHD), specifically in the modulation of arousal (di Michele, Prichep et al. 2005, Rowe, Robinson et al. 2005). Although highly speculative, this fact could explain the association that we found in males between increased QUIN-immunoreactivity and greater distance covered in the OF.

$\underline{4.3 \text { Correlation between the number of QUIN-positive cells in the CC and }}$ QUIN-immunoreactivity in the TRN

Interestingly, we found that the number of QUIN-positive cells in the CC was correlated to the degree of QUIN-immunoreactivity in the TRN. Evidence shows that the $\mathrm{CC}$ and the TRN are anatomically connected. The TRN receives projections from both the thalamus and cortex (Pinault 
2004, Çavdar, Onat et al. 2008), which supports our finding of a correlation in QUIN-immunoreacitivity between the CC and the TRN. In this study, the behavioural screening did not specifically address behavioral phenotypes directly related to the CC and TRN. In addition, the fact that no correlations were observed between QUIN-immunoreactivity in these two brain areas and behavioural performance in the FST in naïve mice, does not exclude a functional role for QUIN in the CC and TRN under challenging conditions, such as chronic inflammation or stress. Evidently, it would be highly interesting to more closely assess the exact role of QUIN function within the brain in view of e.g. emotion, learning, memory, navigation and hyperactivity. Moreover, as we already observe a role for QUIN in the CC and in the TRN in naïve mice, further research should also address the role of QUIN in these brain regions in models of stress and/or inflammation linked to depression.

\section{$\underline{4.4 \text { Concluding remarks }}$}

As mentioned above, several neurotoxic effects have been attributed to altered QUIN levels, after injury or in patients with psychiatric disorders. In these cases, QUIN-immunoreactivity has mainly been reported in the hippocampus and cortex (Guillemin, Brew et al. 2005, Chung, Leung et al. 2009, Rahman, Ting et al. 2009, Chen, Stankovic et al. 2010). However, physiological levels of QUIN are required in normal, healthy conditions to produce nicotinamide adenine dinucleotide(NAD), which is needed for any cell to function properly (Myint 2012). In control samples of a number of studies, controversial results have been reported after evaluation of 
QUIN-immunoreactivity. No QUIN-immunoreactivity was found in the rat cortex (Chung, Leung et al. 2009) and brain parenchyma of control rats (Moffett, Espey et al. 1993). In addition, another study showed no QUINimmunoreactivity in the brains of sham-operated gerbils (Baratte, Molinari et al. 1998). On the other hand, when using human post-mortem control brain tissue, weak QUIN-immunoreactivity was detected in temporal neocortical and hippocampal areas (Guillemin, Brew et al. 2005, Guillemin, Cullen et al. 2007). In addition, QUIN-immunoreactivity has been shown in the cortex of control human brain tissue (Steiner, Walter et al. 2011). Amongst other things, species and strain differences as well as tissue processing and the use of different antibodies for staining for QUIN may explain the diversity of the results obtained.

We conclude that QUIN expression, particularly observed in the CC and TRN of naïve mice, and strongly correlated with locomotor activity in males, may play an important role at the level of the TRN in regulating motor behaviour. Evidently, it would be of great value to further explore the exact role of QUIN-immunoreactivity in mice in order to understand its neurotoxic effects in cases of injury, inflammation or in patients with psychiatric disorders. 


\section{Acknowledgements}

This study was supported by the Commission of European Communities 7th Framework Programme collaborative project MOODINFLAME (grant No. 22963) and partially by FP 7 Marie-Curie IAAP Project Psych-Aid (Grant No. 286334). We would like to thank Advanced Pratical Diagnostics, (ApDia) for providing the anti-QUIN antibody. The authors are grateful to thank Andreas Weiss, Andrea Millet, Waldtraud Stettinger, Prof. Markus Schwarz and the staff members from animal experiment laboratory of Ludwig-Maximilian University of Munich for their guidance and help in the performance of the experiments. 


\section{$\underline{\text { References }}$}

Asp, L., A. S. Johansson, A. Mann, B. Owe-Larsson, E. M. Urbanska, T. Kocki, M. Kegel, G. Engberg, G. B. Lundkvist and H. Karlsson (2011). "Effects of proinflammatory cytokines on expression of kynurenine pathway enzymes in human dermal fibroblasts." J Inflamm (Lond) 8: 25.

Baratte, S., A. Molinari, O. Veneroni, C. Speciale, L. Benatti and P. Salvati (1998). "Temporal and spatial changes of quinolinic acid immunoreactivity in the gerbil hippocampus following transient cerebral ischemia." Brain Res Mol Brain Res 59(1): 50-57.

Beninger, R. J., K. Jhamandas, R. J. Boegman and S. R. el-Defrawy (1986). "Kynurenic acid-induced protection of neurochemical and behavioural deficits produced by quinolinic acid injections into the nucleus basalis of rats." Neurosci Lett 68(3): 317-321.

Busse, M., S. Busse, A. M. Myint, T. Gos, H. Dobrowolny, U. J. Muller, B. Bogerts, H. G. Bernstein and J. Steiner (2014). "Decreased quinolinic acid in the hippocampus of depressive patients: evidence for local anti-inflammatory and neuroprotective responses?" Eur Arch Psychiatry Clin Neurosci.

Çavdar, S., F. Y. Onat, Y. Ö. Çakmak, H. R. Yananli, M. Gülçebi and R. Aker (2008). "The pathways connecting the hippocampal formation, the thalamic reuniens nucleus and the thalamic reticular nucleus in the rat." Journal of anatomy 212(3): 249-256.

Chen, Y., R. Stankovic, K. M. Cullen, V. Meininger, B. Garner, S. Coggan, R. Grant, B. J. Brew and G. J. Guillemin (2010). "The kynurenine pathway and inflammation in amyotrophic lateral sclerosis." Neurotox Res 18(2): 132142.

Chung, R. S., Y. K. Leung, C. W. Butler, Y. Chen, E. D. Eaton, M. W. Pankhurst, A. K. West and G. J. Guillemin (2009). "Metallothionein treatment attenuates microglial activation and expression of neurotoxic quinolinic acid following traumatic brain injury." Neurotox Res 15(4): 381-389.

Dalley, J. W., R. N. Cardinal and T. W. Robbins (2004). "Prefrontal executive and cognitive functions in rodents: neural and neurochemical substrates." Neurosci Biobehav Rev 28(7): 771-784.

Dang, Y., W. E. Dale and O. R. Brown (2000). "Comparative effects of oxygen on indoleamine 2,3-dioxygenase and tryptophan 2,3-dioxygenase of the kynurenine pathway." Free Radic Biol Med 28(4): 615-624.

di Michele, F., L. Prichep, E. R. John and R. J. Chabot (2005). "The neurophysiology of attention-deficit/hyperactivity disorder." Int J Psychophysiol 58(1): 8193. 
Gos, T., A.-M. Myint, K. Schiltz, G. Meyer-Lotz, H. Dobrowolny, S. Busse, U. J. Müller, C. Mawrin, H.-G. Bernstein, B. Bogerts and J. Steiner (2014). "Reduced microglial immunoreactivity for endogenous NMDA receptor agonist quinolinic acid in the hippocampus of schizophrenia patients." Brain, Behavior, and Immunity 41(0): 59-64.

Guillemin, G. J., B. J. Brew, C. E. Noonan, O. Takikawa and K. M. Cullen (2005). "Indoleamine 2,3 dioxygenase and quinolinic acid immunoreactivity in Alzheimer's disease hippocampus." Neuropathol Appl Neurobiol 31(4): 395-404.

Guillemin, G. J., K. M. Cullen, C. K. Lim, G. A. Smythe, B. Garner, V. Kapoor, O. Takikawa and B. J. Brew (2007). "Characterization of the kynurenine pathway in human neurons." J Neurosci 27(47): 12884-12892.

Guillemin, G. J., G. Smythe, O. Takikawa and B. J. Brew (2005). "Expression of indoleamine 2,3-dioxygenase and production of quinolinic acid by human microglia, astrocytes, and neurons." Glia 49(1): 15-23.

Gulaj, E., K. Pawlak, B. Bien and D. Pawlak (2010). "Kynurenine and its metabolites in Alzheimer's disease patients." Adv Med Sci 55(2): 204-211.

Hadland, K. A., M. F. Rushworth, D. Gaffan and R. E. Passingham (2003). "The effect of cingulate lesions on social behaviour and emotion." Neuropsychologia 41(8): 919-931.

Heyes, M. P., K. Saito, J. S. Crowley, L. E. Davis, M. A. Demitrack, M. Der, L. A. Dilling, J. Elia, M. J. Kruesi, A. Lackner and et al. (1992). "Quinolinic acid and kynurenine pathway metabolism in inflammatory and non-inflammatory neurological disease." Brain 115 ( Pt 5): 1249-1273.

Kozlovskiy, S. A., A. V. Vartanov, E. Y. Nikonova, M. M. Pyasik and B. M. Velichkovsky (2012). "The cingulate cortex and human memory processes." Psychol. Russia State Art 5: 231-243.

Kronfol, Z. and D. G. Remick (2000). "Cytokines and the brain: implications for clinical psychiatry." Am J Psychiatry 157(5): 683-694.

Lam, Y.-W. and S. M. Sherman (2011). "Functional Organization of the Thalamic Input to the Thalamic Reticular Nucleus." The Journal of Neuroscience 31(18): 6791-6799.

Maddock, R. J. (1999). "The retrosplenial cortex and emotion: new insights from functional neuroimaging of the human brain." Trends Neurosci 22(7): 310316.

Maes, M. (2008). "The cytokine hypothesis of depression: inflammation, oxidative $\&$ nitrosative stress (IO\&NS) and leaky gut as new targets for adjunctive treatments in depression." Neuro Endocrinol Lett 29(3): 287-291.

Maes, M., R. Yirmyia, J. Noraberg, S. Brene, J. Hibbeln, G. Perini, M. Kubera, P. Bob, B. Lerer and M. Maj (2009). "The inflammatory \& neurodegenerative 
(I\&ND) hypothesis of depression: leads for future research and new drug developments in depression." Metab Brain Dis 24(1): 27-53.

Misztal, M., J. Skangiel-Kramska, G. Niewiadomska and W. Danysz (1996). "Subchronic intraventricular infusion of quinolinic acid produces working memory impairment--a model of progressive excitotoxicity." Neuropharmacology 35(4): 449-458.

Moffett, J. R., M. G. Espey, S. J. Gaudet and M. A. Namboodiri (1993). "Antibodies to quinolinic acid reveal localization in select immune cells rather than neurons or astroglia." Brain Res 623(2): 337-340.

Myint, A. M. (2012). "Kynurenines: from the perspective of major psychiatric disorders." FEBS Journal 279(8): 1375-1385.

Myint, A. M., Y. K. Kim, R. Verkerk, S. Scharpe, H. Steinbusch and B. Leonard (2007). "Kynurenine pathway in major depression: evidence of impaired neuroprotection." J Affect Disord 98(1-2): 143-151.

Nemeth, H., J. Toldi and L. Vecsei (2005). "Role of kynurenines in the central and peripheral nervous systems." Curr Neurovasc Res 2(3): 249-260.

Németh, H., J. Toldi and L. Vécsei (2006). Kynurenines, Parkinson's disease and other neurodegenerative disorders: preclinical and clinical studies. Parkinson's Disease and Related Disorders. P. Riederer, H. Reichmann, M. B. H. Youdim and M. Gerlach, Springer Vienna. 70: 285-304.

Nilsson, L. K., K. R. Linderholm, G. Engberg, L. Paulson, K. Blennow, L. H. Lindström, C. Nordin, A. Karanti, P. Persson and S. Erhardt "Elevated levels of kynurenic acid in the cerebrospinal fluid of male patients with schizophrenia." Schizophrenia Research 80(2): 315-322.

Pinault, D. (2004). "The thalamic reticular nucleus: structure, function and concept." Brain Res Brain Res Rev 46(1): 1-31.

Power, B. D. and J. Mitrofanis (2001). "Zona incerta: substrate for contralateral interconnectivity in the thalamus of rats." Journal of Comparative Neurology 436(1): 52-63.

Rahman, A., K. Ting, K. M. Cullen, N. Braidy, B. J. Brew and G. J. Guillemin (2009). "The excitotoxin quinolinic acid induces tau phosphorylation in human neurons." PLoS One 4(7): e6344.

Raison, C. L., R. Dantzer, K. W. Kelley, M. A. Lawson, B. J. Woolwine, G. Vogt, J. R. Spivey, K. Saito and A. H. Miller (2010). "CSF concentrations of brain tryptophan and kynurenines during immune stimulation with IFN-alpha: relationship to CNS immune responses and depression." Mol Psychiatry 15(4): 393-403.

Rowe, D. L., P. A. Robinson, I. L. Lazzaro, R. C. Powles, E. Gordon and L. M. Williams (2005). "Biophysical modeling of tonic cortical electrical activity in attention deficit hyperactivity disorder." Int J Neurosci 115(9): 1273-1305. 
Shear, D. A., J. Dong, K. L. Haik-Creguer, T. J. Bazzett, R. L. Albin and G. L. Dunbar (1998). "Chronic administration of quinolinic acid in the rat striatum causes spatial learning deficits in a radial arm water maze task." Exp Neurol 150(2): 305-311.

Steiner, J., M. Walter, T. Gos, G. Guillemin, H.-G. Bernstein, Z. Sarnyai, C. Mawrin, R. Brisch, H. Bielau, L. zu Schwabedissen, B. Bogerts and A.-M. Myint (2011). "Severe depression is associated with increased microglial quinolinic acid in subregions of the anterior cingulate gyrus: Evidence for an immunemodulated glutamatergic neurotransmission?" Journal of Neuroinflammation 8(1): 94.

Vann, S. D., J. P. Aggleton and E. A. Maguire (2009). "What does the retrosplenial cortex do?" Nat Rev Neurosci 10(11): 792-802.

Vogt, B. A., L. Vogt and N. Farber (2004). "Cingulate cortex and disease models." The rat nervous system 3: 705-727.

Zhang, J.-M. and J. An (2007). "Cytokines, Inflammation and Pain." International anesthesiology clinics 45(2): 27-37. 


\section{Chapter 5}

\section{Effects of prenatal Poly I:C exposure on global histone deacetylase (HDAC) and DNA methyltransferase (DNMT) activity in the mouse brain}

Yara Pujol Lopez; Gunter Kenis; Waldtraud Stettinger; Karin Neumeier; Sylvia de Jonge; Harry WM Steinbusch; Peter Zill; Daniel LA van den Hove*; Aye M Myint*

* These authors contributed equally to this work 


\section{Abstract}

The aim of our study was to investigate the brain-specific epigenetic effects on global enzymatic histone deacetylase (HDAC) and DNA methyltransferase (DNMT) activity after prenatal exposure to maternal immune challenge by polyinosinic:polycytidylic acid (Poly I:C) at gestational day (GD) 17 in C57BL/6JRccHsd mouse offspring. Pregnant mice were randomly divided into 2 groups, receiving either $5 \mathrm{mg} / \mathrm{kg}$ Poly l:C or phosphate buffered saline (PBS) intravenously at GD 17. Subsequently, the effects on whole brain enzymatic HDAC and DNMT activity and the protein levels of various HDAC isoforms were assessed in the offspring. Overall, a significant sex $x$ treatment interaction effect was observed after prenatal exposure to maternal immune challenge by Poly I:C, indicative of increased global HDAC activity particularly in female offspring from mothers injected with Poly I:C when compared to controls. Results on the levels of specific HDAC isoforms suggested that neither differences in the levels of HDAC1, HDAC2, HDAC3, HDAC4 or HDAC6 could explain the increased global HDAC activity observed in female Poly I:C offspring. In conclusion, we show that Poly I:C administration to pregnant mice alters global brain HDAC, but not DNMT activity in adult offspring, whereas it is still unclear which specific HDAC(s) mediate(s) this effect. These results indicate the necessity for further research on the epigenetic effects of Poly I:C. 


\section{Introduction}

Infection during pregnancy and its associated maternal immune response may negatively impact upon offspring brain development (Gilmore and Jarskog 1997, Nawa, Takahashi et al. 2000), thereby increasing the risk to develop psychopathology later in life (Fatemi, Reutiman et al. 2008, Zhou 2012). The exact mechanisms by which prenatal maternal inflammation impacts upon offspring brain development remain to be elucidated though.

Several studies examined the effects of maternal infection during gestation in animal models using different triggers such as lipopolysaccharide (LPS) (Borrell, Vela et al. 2002, Golan, Lev et al. 2005), the human influenza virus (Fatemi, Reutiman et al. 2008) and polyinosinic:polycytidylic acid (Poly I:C). Poly $\mathrm{I}: \mathrm{C}$ is a synthetic double stranded RNA frequently used as a viral mimetic that induces the release of pro-inflammatory cytokines (Doukas, Cutler et al. 1994, Snell, Chernyshev et al. 1997). As such, prenatal Poly I:C injection to the dams has been shown to induce various cognitive and behavioural changes in adult offspring (Ozawa, Hashimoto et al. 2006) including impairments in exploratory behaviour, prepulse inhibition, latent inhibition and spatial working memory (Meyer, Feldon et al. 2005). Moreover, moderate to severe cell loss was observed in the adult hippocampal CA1, CA3 and dentate gyrus after prenatal maternal exposure to Poly I:C (Zuckerman, Rehavi et al. 2003). As Poly I:C is known to induce a short-lasting immune response, the timing of the maternal inflammatory response can be accurately linked to specific windows during foetal development (Zuckerman, Rehavi et al. 2003). Interestingly, when Poly I:C was injected at 
GD 6 or GD 9, impaired latent inhibition was observed in the offspring, whereas this effect was less pronounced when Poly I:C was injected at GD 13 and was even absent when injection occurred at GD 17 (Meyer, Feldon et al. 2006). In the literature, most of the studies use Poly I:C injection to the pregnant dams at GD 9, which roughly corresponds to the end of the first trimester of human pregnancy. Prenatal maternal infection during this period has mainly been associated with the development of schizophrenia (Brown, Begg et al. 2004, Li, Cheung et al. 2009, Sørensen, Mortensen et al. 2009). However, prenatal maternal immune challenge with Poly I:C at GD 17 has been linked primarily to the development of depressive-like behaviour in the offspring (Enayati, Solati et al. 2012, Babri, Doosti et al. 2014).

Despite the fact that this field of research has received increased attention, the exact mechanisms behind the association between maternal infection during pregnancy and altered brain development in the offspring have still not been elucidated. One likely candidate mechanism involved is epigenetic programming, which is known to play a critical role during fetal development. Furthermore, epigenetic processes modulate several functions in the adult nervous system, such as adult neurogenesis, synaptic plasticity, as well as cognition and emotionality (Ravi and Kannan 2013). The most well-known epigenetic processes include DNA methylation, histone modifications and noncoding RNAs. Epigenetic changes result in chromatin reorganization and thus regulate gene expression during e.g. cellular differentiation. More specifically, histone deacetylation, which generally leads to the compaction of chromatin and transcriptional repression, has 
recently been linked to the pathophysiology of various psychiatric disorders. For example, changes in histone acetylation have been reported in animal models of depression and stress exposure (Yamawaki, Fuchikami et al. 2012). In addition to histone modifications, DNA methylation has also been shown to be involved in the development of depressive-like behaviours (Sales, Biojone et al. 2011), whereas both histone deacetylase (HDAC) and DNA methyltransferase (DNMT) inhibitors have been reported to exert antidepressant effects (Sales, Biojone et al. 2011, Yamawaki, Fuchikami et al. 2012). In view of prenatal infection, hypoacetylation of histones $\mathrm{H} 3$ and $\mathrm{H} 4$ has been observed in the cortex of juvenile offspring prenatally exposed to Poly I:C (Tang, Jia et al. 2013). Moreover, HDAC inhibitors are known to impact upon the transcriptional regulation of cytokines, immunologic signaling pathways and the inflammatory response (Qureshi and Mehler 2011). To date, the epigenetic effects of prenatal maternal Poly I:C exposure at GD 17 -which, as stated above, links more closely to the development of a depressive-like phenotype- have not yet been studied.

In the present study, we examined the brain-specific global epigenetic effects of prenatal maternal immune challenge using viral mimetic Poly I:C at GD 17 in C57BL/6JRccHsd mouse offspring. We hypothesize that prenatal maternal Poly I:C exposure would lead to increased global HDAC and DNMT activity, which represents a molecular switch modulating the alterations in offspring brain development in response to prenatal maternal inflammation. 


\section{Material and methods}

2.1 Animals

Experiments were conducted in accordance with permissions from the government and the veterinarian administration of Oberbayern (AZ: 55.21-54-2531-61-10). This study used C57BL/6JRccHsd mice obtained from Harlan Laboratories (Eystrup, Germany). Mice were housed 4 per cage in single-sex groups in individually ventilated cages (IVC; cage sizes $480 \mathrm{x}$ $375 \times 210 \mathrm{~mm}$ [depth $\mathrm{x}$ width $\mathrm{x}$ high]) under specific pathogen free (SPF) conditions and maintained on a 12/12h light-dark cycle (light on at 12AM) and temperature-controlled environment (relativity humidity $55 \% \pm 5 \%$; temperature: $22^{\circ} \mathrm{C} \pm 2^{\circ} \mathrm{C}$ : room air exchange rate: 15$)$. Breeding food (Ssniff, Germany) and water were allowed ad libitum. Cages were changed every week. After transporting the mice to the local animal facilities, the habituation period lasted 3 weeks. During this period, mice were subcutaneously injected with a transponder for identification purposes. For mating, a male was added to a cage with a female for a period of 3 days. After mating, females were housed individually. Three weeks after birth, offspring were weaned and separated by sex (4 mice/cage). Offspring were chipped when they were 6 weeks old to facilitate their identification. A maximum of 2 mice/sex/litter were used to prevent litter effects (Chapman and Stern 1978).

\subsection{Mating and induction of prenatal infection}

Approximately 8 weeks after birth, male and female offspring were put together for 3 days. At GD17, pregnancy was confirmed by visual 
inspection of the dam in combination with weight gain analysis (increase between day of mating and the day of the injection). Pregnant mice were randomly divided into 2 groups [Poly I:C; $n=5$ ] and phosphate buffered saline [PBS; $n=6]$ ). Dams were injected intravenously with $5 \mathrm{mg} / \mathrm{kg}$ Poly I:C (potassium salt, Sigma Aldrich (Germany)) dissolved in sterile water or an equivalent volume of PBS. Following injection, mice were observed daily until delivery.

\subsection{Enzymatic HDAC and DNMT activity}

At an age of thirteen weeks, brains from 8 male and 9 female mice prenatally exposed to Poly I:C, and 9 male and 9 female offspring from PBS-treated dams were used for epigenetic analysis. For this purpose, mice were anesthetized with a single intraperitoneal injection of Ketamin (Pfizer, Germany) and Xylacin (Bayer, Germany). $0.7 \mathrm{ml}$ 10\% Ketamin and $0.3 \mathrm{ml} 2 \%$ Xylacin were mixed and administered at a dose of $10 \mathrm{ml} / \mathrm{kg}$. Afterwards, brains were collected and stored at $-20^{\circ} \mathrm{C}$ in RNA-Later (Invitrogen, Germany). Half of the brain was used to extract nuclear proteins using a Nuclear Extract Kit (Active Motif, Belgium) and the activities of various HDAC and DNMT enzymes were analyzed with the HDAC Fluorescent Assay Kit and DNMT Activity/Inhibition Assay (Active Motif, Belgium). The fluorescence/optical density was detected with a PolarStar OPTIMA microplate fluorimeter (BMG Labtech). 


\subsection{Western blot}

In order to elucidate which specific HDAC enzyme(s) would mediate the observed increase in overall HDAC activity (see below), a western blotting experiment was performed. For this purpose, the other half of each brain was crushed while frozen and stored at $-80^{\circ} \mathrm{C}$. Tissue was subsequently mechanically homogenized in the Mini-Beadbeater ${ }^{\mathrm{TM}}$ (Biospec, The Netherlands) using lysis buffer (PBS, $1 \%$ Igepal CA-630 ${ }^{\circledR}, 0.1 \%$ triton, $1 \%$ glycerol, 1mM EDTA, 1mM EGTA) and protease inhibitors (Complete Protease Inhibitor Cocktail, Roche Diagnostics, The Netherlands) in MilliQ. Concentration of total protein was measured using the Lowry protein assay (Bio-Rad, The Netherlands). Proteins dissolved in sample buffer (1M Tris $\mathrm{HCl} \mathrm{pH} \mathrm{6.8,} \mathrm{75 \%} \mathrm{glycerol,} \mathrm{SDS,} \beta$-mercaptoehtanol, bromophenol blue in Milli-Q) were denatured by boiling at $100^{\circ} \mathrm{C}$ for 7 minutes. Thirty microgram of protein per lane, in case of HDAC4 and 6, and $100 \mu \mathrm{g}$ for HDAC1, 2 and 3 was separated by 10\% SDS-polyacrylamide gel electrophoresis (SDS-PAGE) for $75 \mathrm{~min}$ at $150 \mathrm{~V}$ and transferred to a nitrocellulose membrane during $90 \mathrm{~min}$ at $100 \mathrm{~V}$ using a Bio-Rad Laboratories Western Blotting system. Next, membranes were rinsed once in PBS and blocked in 1:1 Odyssey Blocking Buffer:PBS (LICOR Biosciences, NE, USA) for 1 hour at room temperature. Following the blocking of the membranes, they were incubated overnight at $4^{\circ} \mathrm{C}$ with the respective monoclonal mouse anti-mouse HDAC1 (1:350), HDAC2 (1:500), HDAC3 (1:350) and monoclonal anti-rabbit HDAC4 (1:500) contained in the HDAC Antibody Sampler Kit (\#9928, Cell Signaling Technology, Inc.) or the monoclonal anti-rabbit HDAC6 (1:500) (\#7612, 
Cell Signaling Technology, Inc.) and 1:1000 diluted monoclonal mouse anti- $\beta$-actin (sc-81178, Santa Cruz Biotechnology) diluted in either 1:1 Odyssey Blocking Buffer:PBS (HDAC1, 3, 4) or 1:1 Blocking buffer:PBS + 0,2\% Tween-20 (HDAC2 and 6). After incubation with the primary antibody, membranes were rinsed once with PBS:Tween and twice with PBS and then incubated with the corresponding secondary antibody, i.e., Alexa Fluor ${ }^{\circledR} 680$ donkey anti-mouse (1:10000) IgG or Alexa Fluor ${ }^{\circledR} 800$ goat anti-rabbit IgG (1:5000; LI-COR Biosciences) diluted in 1:1 Blocking buffer:PBS for 1 hour at room temperature. Membranes were rinsed again as explained before and bands were visualized by enhanced chemiluminescence detection using an Odyssey Scanner and the Odyssey Infrared Imaging System v2.1 software (LI-COR Biosciences). Bands were quantified with the ImageJ software (NIH, USA) using mean intensity and normalizing for $\beta$-actin expression.

\subsection{Statistical analysis}

The effects of prenatal maternal Poly I:C exposure on enzymatic HDAC and DNMT activity and protein levels of each HDAC isoform were first subjected to a two-way analysis of variance (ANOVA; treatment $x$ sex) with IBM SPSS Statistics version 20 (SPSS Inc., USA). Significant interaction effects were analyzed in more detail using post-hoc LSD tests. The level of significance was set at $p<0.05$ in all cases. Graphs were designed with GraphPad Prism 6. 


\section{Results}

\subsection{Global enzymatic activity}

Regarding HDAC activity, a significant sex $x$ treatment interaction effect was found $\left(F_{3,31}=5.477 ; p<0.05\right)$ (see Figure 1$)$. Post hoc LSD tests indicated that HDAC activity tended to be increased in female offspring from mothers injected with Poly I:C $(p=0.064)$ compared to those injected with PBS. No overall effects were found for sex or treatment $\left(F_{3,31}=0.884\right.$ and $F_{3,31}=0.110$ respectively; both $p>0.05$ ). For total DNMT activity, no significant effects were detected $\left(F_{3,31}=1.676, F_{3,31}=0.826, F_{3,31}=0.159\right.$ for sex, treatment and their interaction, respectively; all $p>0.05$ ) (see Figure 1).
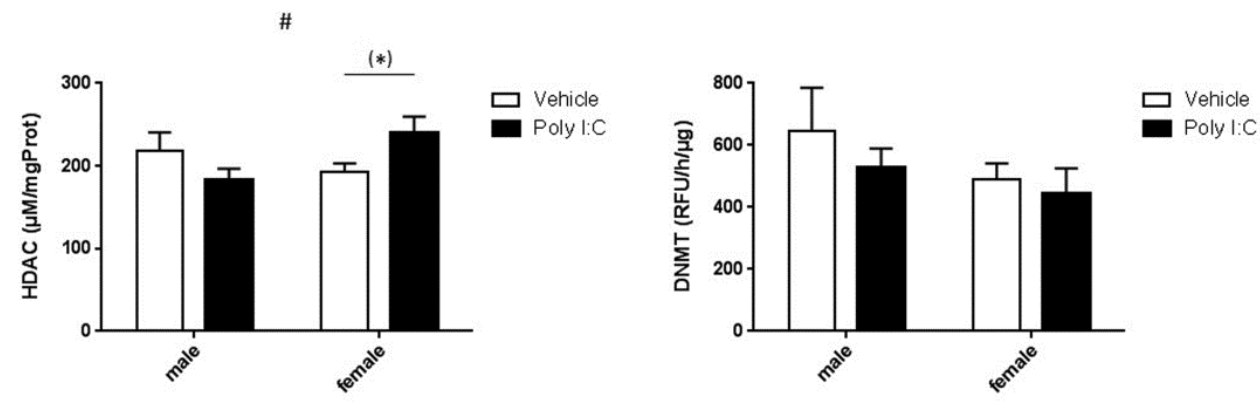

Figure 1. HDAC and DNMT activity levels in mouse offspring. Bars represent means + S.E.M, $\# p<0.05$ (ANOVA, sex $x$ treatment), $\left({ }^{*}\right) p=0.064$ (LSD). Vehicle male $n=9$, vehicle female $n=9$, Poly I:C male $n=8$, Poly $\mathrm{I}: \mathrm{C}$ female $n=9$. For total DNMT activity no effects were detected $\left(F_{3,31}=1.676, F_{3,31}=0.826\right.$, $F_{3,31}=0.159$; all $\left.p>0.05\right)$.

\subsection{HDAC isoforms}

Overall, a sex effect was observed with reduced HDAC1 levels in female versus male mice $\left(F_{3,31}=4.389 ; p<0.05\right)$. Furthermore, a tendency for an overall treatment effect was shown for HDAC6 with increased protein 
levels in Poly I:C offspring when compared to control offspring $\left.\left(F_{3,29}=4.061 ; p=0.053\right)\right)$. No effect was found for HDAC2, HDAC3, and HDAC4 (all $F_{3,31}<3 ; p>0.05$ ) (see Table 1 and Figure 2).

Table 1. Mean and S.E.M for each condition for the correspondent HDAC

\begin{tabular}{|c|c|c|c|c|c|c|c|c|c|}
\cline { 2 - 9 } \multicolumn{1}{c|}{} & \multicolumn{2}{c|}{$\begin{array}{c}\text { male } \\
\text { vehicle }\end{array}$} & \multicolumn{2}{c|}{$\begin{array}{c}\text { male } \\
\text { Poly I:C }\end{array}$} & \multicolumn{2}{c|}{$\begin{array}{c}\text { female } \\
\text { vehicle }\end{array}$} & \multicolumn{2}{c|}{$\begin{array}{c}\text { female } \\
\text { Poly I:C }\end{array}$} & effects \\
\cline { 2 - 10 } & M & SEM & M & SEM & M & SEM & M & SEM & \\
\hline HDAC1 & 0.1655 & 0.0402 & 0.2027 & 0.0510 & 0.1195 & 0.0350 & 0.0875 & 0,0264 & sex effect $p<0.05$ \\
\hline HDAC2 & 0.2518 & 0.0353 & 0.1874 & 0.0285 & 0.2362 & 0.0278 & 0.2620 & 0.0475 & \\
\hline HDAC3 & 0.0735 & 0.0262 & 0.0502 & 0.0323 & 0.0506 & 0.0125 & 0.0501 & 0.0313 & \\
\hline HDAC4 & 0.1303 & 0.0322 & 0.1194 & 0.0335 & 0.1005 & 0.0241 & 0.1178 & 0.0296 & \\
\hline
\end{tabular}



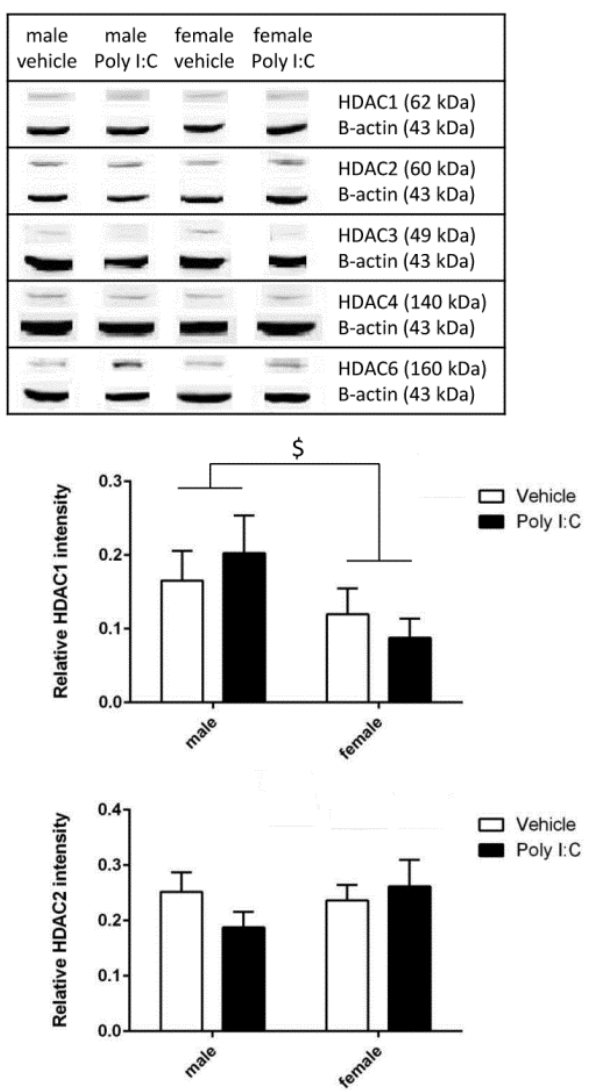
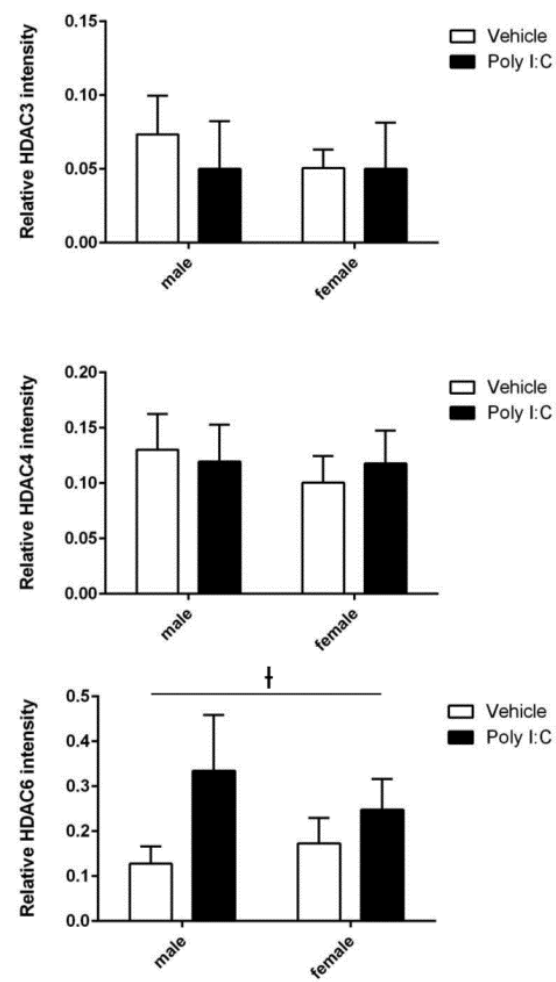

Figure 2. Levels of HDAC1, HDAC2, HDAC3, HDAC4 and HDAC6 proteins in brain homogenates and corresponding quantification. Bars represent means + S.E.M, $\$ p<0.05$ (ANOVA, sex), $+p<0.1$ (ANOVA, treatment). HDAC1-5: Vehicle male $n=9$, vehicle female $n=9$, Poly I:C male $n=8$, Poly I:C female $n=9$. HDAC 6: Vehicle male $n=9$, vehicle female $n=9$, Poly I:C male $n=6$, Poly I:C female $n=9$.

\section{Discussion}

In the present paper, we report that prenatal maternal Poly I:C exposure in the mouse modulates global brain HDAC activity, but not DNMT activity in adult offspring. HDAC activity was particularly increased in female offspring exposed to prenatal maternal Poly I:C when compared to controls, suggesting that epigenetic programming may, at least in part, mediate the long-term effects of prenatal maternal infection. 
Exposure to pathogens triggers an inflammatory response with concomitant up-regulation of pro-inflammatory cytokines. Interestingly, next to the fact that HDACs were shown to regulate gene transcription of cytokines (Lu, Sun et al. 2005, Villagra, Sotomayor et al. 2009), cytokines have recently also been shown to regulate the activity of HDACs. For example, activation of pro-inflammatory cytokines, such as tumour necrosis factor- $\alpha$ (TNF- $\alpha$ ) and interferon- $\gamma$ (IFN- $\gamma)$, produces HDAC1 ubiquitinization and proteosomal degradation (Villagra, Sotomayor et al. 2009). Apart from the modulation of innate immune system function, HDACs also regulate adaptive immunity (Shakespear, Halili et al. 2011). Depletion of both HDAC1 and HDAC2, specifically in B cells leads to blocking of early B cell development (Yamaguchi, Cubizolles et al. 2010). Over the last years, increased evidence linked histone acetylation to the neurobiology and pharmacology of mood disorders (Zarate, Singh et al. 2006). For instance, chronic defeat stress resulted in an impaired behavioural response and in the downregulation of brain-derived neurotrophic factor (BDNF) transcripts III and IV, which was reversed by imipramine. Interestingly, this antidepressant also increased histone acetylation at these promoters and was associated with a downregulation of HDAC5 (Tsankova, Berton et al. 2006). Vice versa, HDAC inhibitors have also been shown to possess antidepressant properties (Covington, Maze et al. 2009, Grayson, Kundakovic et al. 2010). For example, administration of the HDAC inhibitor sodium butyrate alone or in combination with fluoxetine decreased immobility scores in the tail suspension test, a measure for behavioural despair, in mice (Schroeder, Lin et al. 2007). However, these studies used naïve mice that were not exposed 
to a prenatal immune challenge. As mentioned above, the effects produced by prenatal maternal infection are depending on the timing of exposure during gestation and their behavioural expression may also depend on the age of the offspring. For instance, after Poly I:C injection at GD 9, global histone $\mathrm{H} 3$ and $\mathrm{H} 4$ hypoacetylation was shown in in the cortex of juvenile but not adult offspring (Tang, Jia et al. 2013). Our results are partially consistent with this study, e.g. we found that HDAC activity was increased in offspring that were exposed to prenatal maternal Poly I:C (at GD 17), an effect that seemed to be particularly pronounced in females. However, our findings were observed in adult offspring, whereas Tang and colleagues detected hypoacetylation only in juvenile offspring, a difference that may be explained by the different timing of prenatal maternal Poly I:C exposure.

We hypothesized that the observed increase in global HDAC activity in females, which were prenatally exposed to maternal Poly I:C, would be reflected in enhanced expression of one of the HDAC proteins assessed. However, the western blot showed that none of the HDAC enzymes examined was present in significantly higher levels in Poly I:C versus control female offspring. Although we observed a treatment effect with increased HDAC6 in offspring of mice treated with Poly $1: C$ compared to controls, this effect seemed to be more pronounced in male - and not female - offspring, which does not match the global HDAC activity data.

In the brain, HDAC6 is involved in protein aggregate elimination, in neuronal oxidative stress and in the mitochondrial transport (Simoes-Pires, Zwick et al. 2013). Interestingly, HDAC6 deficiency in mice resulted in hyperactivity 
and reduced anxiety, and depression-like behavior. Similarly, administration of an HDAC6 inhibitor also had an antidepressant-like effect, suggesting a role for HDAC6 in the expression of emotional behaviours (Fukada, Hanai et al. 2012). As commented above, prenatal maternal Poly I:C exposure has been associated with the development of depressive-like behaviour in the offspring. Interestingly, we observed that HDAC6 protein levels tended to be increased in Poly I:C offspring when compared to control offspring. Altogether, though purely speculative, these data suggests a role for HDAC6 in mediating the association between prenatal maternal infection and the development of depression in the offspring. Thus, further research investigating this possible role of HDAC6, would be of great value.

In addition, we observed a sex effect with reduced HDAC1 levels in female mice compared to males. Regarding brain functions, HDAC1 is reported as the molecular switch between neuronal survival and death (Bardai, Price et al. 2012) and being considered as a negative regulator of fear extinction in mice (Bahari-Javan, Maddalena et al. 2012) and as a negative regulator of mood (Schroeder, Lewis et al. 2013). No studies have examined sex differences in HDAC1 function in this respect. Thus, future research should address possible variations in HDAC1 levels between sexes.

A feasible explanation for the discrepancy observed between the global HDAC activity and the protein levels of several HDAC isoforms in the present study is that one or more other HDAC isoform(s) than those assessed using western blotting is/are involved in mediating the observed increased in global HDAC activity. HDACs are part of a large family of proteins, with 11 members identified to date. We examined HDAC1, HDAC2, HDAC3, HDAC4 
and HDAC6, because of the evidence showing their association with mental (dys)function (McQuown, Barrett et al. 2011, Bahari-Javan, Maddalena et al. 2012, Fukada, Hanai et al. 2012, Morris, Mahgoub et al. 2013, Sarkar, Chachra et al. 2014). A viable candidate that may contribute to the effect found in HDAC global activity is HDAC5, which has been shown to be involved in inflammation, specifically in activated monocytes and macrophages (Cantley and Haynes 2013). Moreover, decreased HDAC5 levels in the hippocampus has been associated to the antidepressant effects of imipramine in mice models, as explained above (Tsankova, Berton et al. 2006). We initially included HDAC5 in the present study, but after trying numerous different antibodies in several organ tissues, we were not able to detect a specific band indicating HDAC5. Another, completely different possible explanation for the observed discrepancy may lie in the fact that an increased enzymatic activity does not necessarily imply an increase in the level of the associated protein(s). Clearly, this issue awaits further investigation. 


\section{$\underline{4.1 \text { Conclusions }}$}

Altogether, this study indicates that prenatal maternal Poly I:C exposure seems to increase global HDAC, but not DNMT activity, particularly in female offspring. Evidently, it would be of great value to further explore the exact role of epigenetic programming in response to prenatal exposure to maternal immune challenge using Poly $\mathrm{I}: \mathrm{C}$ in order to understand the mechanism underlying the association between prenatal infection/inflammation and adult psychopathology.

\subsection{Limitations}

It should be noted that mice were anesthetized in this study in order to comply with the approval of the ethics committee. Although the time window between exposure and actual euthanasia was extremely short in our case, we cannot fully exclude the possibility that the use of anesthesia may have influenced epigenetic processes (Lirk, Fiegl et al. 2014). In addition, it should be noted that for assessing both HDAC levels and activity, whole brain homogenates were used. It would evidently be of additional interest to evaluate specific brain areas and cell-type-specific epigenetic changes. 


\section{Acknowledgements}

The study was supported by the Commission of European Communities $7^{\text {th }}$ Framework Programme collaborative project "MOODINFLAME" (Grant No. 22963).

The authors would like to thank Andreas Weiss, Andrea Millet and Prof. Markus Schwarz, and the staff members from animal experiment laboratory of Ludwig-Maximilian University of Munich for their guidance and help in the performance of the experiments. 


\section{References}

Babri, S., M.-H. Doosti and A.-A. Salari (2014). "Strain-dependent effects of prenatal maternal immune activation on anxiety- and depression-like behaviors in offspring." Brain, Behavior, and Immunity 37(0): 164-176.

Bahari-Javan, S., A. Maddalena, C. Kerimoglu, J. Wittnam, T. Held, M. Bahr, S. Burkhardt, I. Delalle, S. Kugler, A. Fischer and F. Sananbenesi (2012). "HDAC1 regulates fear extinction in mice." J Neurosci 32(15): 5062-5073.

Bardai, F. H., V. Price, M. Zaayman, L. Wang and S. R. D'Mello (2012). "Histone deacetylase-1 (HDAC1) is a molecular switch between neuronal survival and death." J Biol Chem 287(42): 35444-35453.

Borrell, J., J. M. Vela, A. Arevalo-Martin, E. Molina-Holgado and C. Guaza (2002). "Prenatal immune challenge disrupts sensorimotor gating in adult rats. Implications for the etiopathogenesis of schizophrenia." Neuropsychopharmacology 26(2): 204-215.

Brown, A. S., M. D. Begg, S. Gravenstein and et al. (2004). "SErologic evidence of prenatal influenza in the etiology of schizophrenia." Archives of General Psychiatry 61(8): 774-780.

Cantley, M. and D. Haynes (2013). "Epigenetic regulation of inflammation: progressing from broad acting histone deacetylase (HDAC) inhibitors to targeting specific HDACs." Inflammopharmacology 21(4): 301-307.

Chapman, R. H. and J. M. Stern (1978). "Maternal stress and pituitary-adrenal manipulations during pregnancy in rats: effects on morphology and sexual behavior of male offspring." J Comp Physiol Psychol 92(6): 1074-1083.

Covington, H. E., 3rd, I. Maze, Q. C. LaPlant, V. F. Vialou, Y. N. Ohnishi, O. Berton, D. M. Fass, W. Renthal, A. J. Rush, 3rd, E. Y. Wu, S. Ghose, V. Krishnan, S. J. Russo, C. Tamminga, S. J. Haggarty and E. J. Nestler (2009). "Antidepressant actions of histone deacetylase inhibitors." J Neurosci 29(37): 11451-11460.

Doukas, J., A. H. Cutler and J. P. Mordes (1994). "Polyinosinic:polycytidylic acid is a potent activator of endothelial cells." Am J Pathol 145(1): 137-147.

Enayati, M., J. Solati, M. H. Hosseini, H. R. Shahi, G. Saki and A. A. Salari (2012). "Maternal infection during late pregnancy increases anxiety- and depression-like behaviors with increasing age in male offspring." Brain Res Bull 87(2-3): 295-302.

Fatemi, S. H., T. J. Reutiman, T. D. Folsom, H. Huang, K. Oishi, S. Mori, D. F. Smee, D. A. Pearce, C. Winter, R. Sohr and G. Juckel (2008). "Maternal infection leads to abnormal gene regulation and brain atrophy in mouse offspring: Implications for genesis of neurodevelopmental disorders." Schizophrenia Research 99(1-3): 56-70. 
Fukada, M., A. Hanai, A. Nakayama, T. Suzuki, N. Miyata, R. M. Rodriguiz, W. C. Wetsel, T. P. Yao and Y. Kawaguchi (2012). "Loss of deacetylation activity of Hdac6 affects emotional behavior in mice." PLoS One 7(2): e30924.

Gilmore, J. H. and L. F. Jarskog (1997). "Exposure to infection and brain development: cytokines in the pathogenesis of schizophrenia." Schizophr Res 24(3): 365-367.

Golan, H. M., V. Lev, M. Hallak, Y. Sorokin and M. Huleihel (2005). "Specific neurodevelopmental damage in mice offspring following maternal inflammation during pregnancy." Neuropharmacology 48(6): 903-917.

Grayson, D. R., M. Kundakovic and R. P. Sharma (2010). "Is there a future for histone deacetylase inhibitors in the pharmacotherapy of psychiatric disorders?" Mol Pharmacol 77(2): 126-135.

Li, Q., C. Cheung, R. Wei, E. S. Hui, J. Feldon, U. Meyer, S. Chung, S. E. Chua, P. C. Sham, E. X. Wu and G. M. McAlonan (2009). "Prenatal Immune Challenge Is an Environmental Risk Factor for Brain and Behavior Change Relevant to Schizophrenia: Evidence from MRI in a Mouse Model." PLOS ONE 4(7): e6354.

Lirk, P., H. Fiegl, N. C. Weber and M. W. Hollmann (2014). "Epigenetics in the Perioperative Period." Br J Pharmacol.

Lu, J., H. Sun, X. Wang, C. Liu, X. Xu, F. Li and B. Huang (2005). "Interleukin-12 p40 promoter activity is regulated by the reversible acetylation mediated by HDAC1 and p300." Cytokine 31(1): 46-51.

McQuown, S. C., R. M. Barrett, D. P. Matheos, R. J. Post, G. A. Rogge, T. Alenghat, S. E. Mullican, S. Jones, J. R. Rusche, M. A. Lazar and M. A. Wood (2011). "HDAC3 Is a Critical Negative Regulator of Long-Term Memory Formation." The Journal of neuroscience : the official journal of the Society for Neuroscience 31(2): 764-774.

Meyer, U., J. Feldon, M. Schedlowski and B. K. Yee (2005). "Towards an immunoprecipitated neurodevelopmental animal model of schizophrenia." Neuroscience \& Biobehavioral Reviews 29(6): 913-947.

Meyer, U., J. Feldon, M. Schedlowski and B. K. Yee (2006). "Immunological stress at the maternal-foetal interface: a link between neurodevelopment and adult psychopathology." Brain Behav Immun 20(4): 378-388.

Morris, M. J., M. Mahgoub, E. S. Na, H. Pranav and L. M. Monteggia (2013). "Loss of histone deacetylase 2 improves working memory and accelerates extinction learning." J Neurosci 33(15): 6401-6411.

Nawa, H., M. Takahashi and P. H. Patterson (2000). "Cytokine and growth factor involvement in schizophrenia--support for the developmental model." Psychiatry 5(6): 594-603. 
Ozawa, K., K. Hashimoto, T. Kishimoto, E. Shimizu, H. Ishikura and M. Iyo (2006). "Immune activation during pregnancy in mice leads to dopaminergic hyperfunction and cognitive impairment in the offspring: a neurodevelopmental animal model of schizophrenia." Biol Psychiatry 59(6): 546-554.

Qureshi, I. A. and M. F. Mehler (2011). "Epigenetics, nervous system tumors, and cancer stem cells." Cancers (Basel) 3(3): 3525-3556.

Ravi, B. and M. Kannan (2013). "Epigenetics in the nervous system: An overview of its essential role." Indian journal of human genetics 19(4): 384.

Sales, A. J., C. Biojone, M. S. Terceti, F. S. Guimaraes, M. V. Gomes and S. R. Joca (2011). "Antidepressant-like effect induced by systemic and intrahippocampal administration of DNA methylation inhibitors." $\mathrm{Br} \mathrm{J}$ Pharmacol 164(6): 1711-1721.

Sarkar, A., P. Chachra, P. Kennedy, C. J. Pena, L. A. Desouza, E. J. Nestler and V. A. Vaidya (2014). "Hippocampal HDAC4 contributes to postnatal fluoxetineevoked depression-like behavior." Neuropsychopharmacology 39(9): 22212232.

Schroeder, F. A., M. C. Lewis, D. M. Fass, F. F. Wagner, Y.-L. Zhang, K. M. Hennig, J. Gale, W.-N. Zhao, S. Reis, D. D. Barker, E. Berry-Scott, S. W. Kim, E. L. Clore, J. M. Hooker, E. B. Holson, S. J. Haggarty and T. L. Petryshen (2013). "A Selective HDAC $1 / 2$ Inhibitor Modulates Chromatin and Gene Expression in Brain and Alters Mouse Behavior in Two Mood-Related Tests." PLoS ONE 8(8): e71323.

Schroeder, F. A., C. L. Lin, W. E. Crusio and S. Akbarian (2007). "Antidepressant-like effects of the histone deacetylase inhibitor, sodium butyrate, in the mouse." Biol Psychiatry 62(1): 55-64.

Shakespear, M. R., M. A. Halili, K. M. Irvine, D. P. Fairlie and M. J. Sweet (2011). "Histone deacetylases as regulators of inflammation and immunity." Trends Immunol 32(7): 335-343.

Simoes-Pires, C., V. Zwick, A. Nurisso, E. Schenker, P.-A. Carrupt and M. Cuendet (2013). "HDAC6 as a target for neurodegenerative diseases: what makes it different from the other HDACs?" Molecular Neurodegeneration 8(1): 7.

Snell, J. C., O. Chernyshev, D. L. Gilbert and C. A. Colton (1997). "Polyribonucleotides induce nitric oxide production by human monocyte-derived macrophages." J Leukoc Biol 62(3): 369-373.

Sørensen, H. J., E. L. Mortensen, J. M. Reinisch and S. A. Mednick (2009). "Association Between Prenatal Exposure to Bacterial Infection and Risk of Schizophrenia." Schizophrenia Bulletin 35(3): 631-637. 
Tang, B., H. Jia, R. J. Kast and E. A. Thomas (2013). "Epigenetic changes at gene promoters in response to immune activation in utero." Brain Behav Immun 30: 168-175.

Tsankova, N. M., O. Berton, W. Renthal, A. Kumar, R. L. Neve and E. J. Nestler (2006). "Sustained hippocampal chromatin regulation in a mouse model of depression and antidepressant action." Nat Neurosci 9(4): 519-525.

Villagra, A., E. M. Sotomayor and E. Seto (2009). "Histone deacetylases and the immunological network: implications in cancer and inflammation." Oncogene 29(2): 157-173.

Yamaguchi, T., F. Cubizolles, Y. Zhang, N. Reichert, H. Kohler, C. Seiser and P. Matthias (2010). "Histone deacetylases 1 and 2 act in concert to promote the G1-to-S progression." Genes Dev 24(5): 455-469.

Yamawaki, Y., M. Fuchikami, S. Morinobu, M. Segawa, T. Matsumoto and S. Yamawaki (2012). "Antidepressant-like effect of sodium butyrate (HDAC inhibitor) and its molecular mechanism of action in the rat hippocampus." World J Biol Psychiatry 13(6): 458-467.

Zarate, C. A., Jr., J. Singh and H. K. Manji (2006). "Cellular plasticity cascades: targets for the development of novel therapeutics for bipolar disorder." Biol Psychiatry 59(11): 1006-1020.

Zhou, H. (2012). "Maternal Infection and Neurodevelopmental Disorders in the Offspring." American Journal of Immunology 8(1): 10.

Zuckerman, L., M. Rehavi, R. Nachman and I. Weiner (2003). "Immune activation during pregnancy in rats leads to a postpubertal emergence of disrupted latent inhibition, dopaminergic hyperfunction, and altered limbic morphology in the offspring: a novel neurodevelopmental model of schizophrenia." Neuropsychopharmacology 28(10): 1778-1789. 


\section{Chapter 6}

\section{Depressive symptoms during IFN-alpha therapy in humans associated with changes in the kynurenine pathway}

Yara Pujol Lopez; Carsten Leue; Ger Koek; Aye M Myint; Daniel LA van den Hove; Ehsan Pishva; Markus J Schwarz; Jim van Os; Harry WM Steinbusch; Bart PF Rutten; Gunter Kenis 


\section{Abstract}

Although Interferon-alpha (IFN- $\alpha$ ) is effective for the treatment of Hepatitis C, this therapy has been linked to the development of depressive symptoms and major depression in a substantial proportion of patients. This may be mediated by neurotoxic components of the kynurenine pathway. The aim of our study was to investigate the effects of IFN- $\alpha$ therapy for Hepatitis $C$ on the Montgomery-Asberg Depression Rating Scale (MADRS), the Beck Depression Inventory (BDI) and on several enzymes and metabolites of the kynurenine pathway.

Twelve patients diagnosed with Hepatitis C were followed for 48 weeks during treatment with IFN- $\alpha$ and ribavirin. During this period, symptom development was measured and blood samples were obtained at 12 regular time points. Relative gene expression analysis of the above mentioned enzymes was performed by real time quantitative polymerase chain reaction (RT-qPCR) using mRNA derived from blood cells. Metabolites were analysed in serum using a LC-MS/MS system.

During IFN- $\alpha$ therapy, we observed a significant change over time in MADRS and BDI scores. In addition, levels of the neurotoxic metabolite kynurenine (KYN), 3-hydroxykynurenine (3HK), quinolinic acid (QUIN) and the kynurenine/tryptophan (KYN/TRP) ratio were increased over time, while the kynurenic acid/kynurenine (KYNA/KYN) ratio, reflecting neuroprotective properties, was reduced. Regarding the enzymes of the kynurenine pathway kynureninase (KYNase), kynurenine-3-monooxygenase (KMO) and kynurenine aminotransferase (KAT-3) activity were also increased. When assessing the relationship between MADRS scores and metabolites, KYNA 134 
and the KYNA/KYN ratio were negatively associated with MADRS scores. There was no association between enzymes and the MADRS score. Finally, relative expression of KYNase significantly predicted levels of $3 \mathrm{HK}$.

We show that the kynurenine pathway plays and important role in the development of depressive symptoms during IFN- $\alpha$ treatment, with a shift in the balance between neurotoxic and neuroprotective metabolites, as well as increased expression of enzymes regulating the neurotoxic arm of the pathway. 


\section{Introduction}

Interferon-alpha (IFN- $\alpha$ ) is a commonly used cytokine to treat hepatitis C. However, several side effects have been reported, such as fever, fatigue and myalgias in a high percentage of patients, reviewed by (Foster 2010). In addition, full-blown neuropsychiatric syndromes, in particular clinical depression, have been observed (Schaefer, Hinzpeter et al. 2007), and often necessitates interruption or even cessation of the treatment (Debien, De Chouly De Lenclave et al. 2001). Various mechanisms have been suggested to underlie the pro-depressive effects of IFN- $\alpha$ (for review see Hoyo-Becerra, Schlaak et al. 2014). Of particular interest is the induction of proinflammatory cytokines, of which the circulating levels are associated with depressive symptoms during IFN- $\alpha$ treatment (Wichers, Kenis et al. 2007). Pro-inflammatory cytokines and other inflammatory processes can impact on brain function, disturb affect regulation and can lead to depression via diverse mechanisms (Capuron and Miller 2011, Haroon, Raison et al. 2012). Recently, an array of studies points towards an enhanced catabolism of tryptophan along the kynurenine pathway as being a major mediator in the association between inflammation and depression. Cytokines are known to stimulate the enzyme indoleamine 2,3-dioxygenase (IDO) that catabolizes tryptophan to kynurenine (Myint 2012). Thus, an increase in cytokines will result in a reduction of tryptophan (TRP) and will increase kynurenine (KYN). Concomitantly serotonin synthesis will be compromised because of reduced TRP availability (Asnis and De La Garza 2006). KYN can be further metabolized either to kynurenic acid (KYNA) by kynurenine aminotransferases (KAT) -1, KAT-2 and KAT-3, or to quinolinic acid (QUIN) 136 
through several enzymatic reactions. First, KYN is metabolized to 3hydroxykynurenine (3HK) by kynurenine-3-monooxygenase (KMO). Next, $3 \mathrm{HK}$ is further degraded to 3-hydroxyanthranilic acid (3HAA) by kynureninase (KYNase). Finally, 3HAA is degraded into QUIN by 3hydroxyanthranilic acid oxygenase (3HAO). QUIN is further catabolized into nicotinamide adenine dinucleotide (NAD) by quinolinate phosphoribosyl transferase (QPRT) (Bender and McCreanor 1985, Heyes, Achim et al. 1996). Activation of IDO is hypothesized to play an important role in the development of major depression, either via TRP depletion and the consequent reduction in the synthesis of serotonin, or via activation of the KYN pathway and the synthesis of the KYN metabolites (Christmas, Potokar et al. 2011). Some of these, e.g. QUIN, are known to exert neurotoxic effects (Garthwaite and Garthwaite 1987, Khaspekov, Kida et al. 1989). Several studies have evaluated the levels of TRP, KYN and serotonin in depressed patients, but reported controversial results (Hughes, Carballedo et al. 2012, Myint, Bondy et al. 2013).

To our knowledge, in IFN- $\alpha$ induced depression only IDO activity has been analyzed from the several enzymes forming part of the KYN pathway. Its activity was assessed by means of the KYN/TRP ratio (Capuron, Neurauter et al. 2003, Comai, Cavalletto et al. 2011). Gene expression levels of enzymes in the KYN pathway have not yet been examined. The present study was undertaken to longitudinally evaluate: 1 ) the serum levels of TRP, KYN, $3 \mathrm{HK}$, KYNA, QUIN, and 5-Hydroxyindoleacetic acid (5-HIAA) and their ratios, and 2 ) the gene expression levels of the KYN pathway enzymes IDO-1, KAT-1, and 
KAT-3, KMO, KYNase, 3HAO and QPRT in blood of Hepatitis C patients treated with IFN- $\alpha$. The metabolite ratios were calculated as indicators of IDO-activity and activation of the KYN pathway (KYN/TRP), activation of the neurotoxic arm (QUIN/KYN) and activation of the neuroprotective arm (KYNA/KYN) of the pathway. Finally, association of metabolites and enzyme expression levels with depressive symptoms were examined. To our knowledge, this is the most comprehensive study on the KYN pathway in IFN$\alpha$ induced depression, and the first to examine the enzymes involved. We hypothesize that treatment with IFN- $\alpha$ induces depressive symptoms, which are associated with changes in the levels of the metabolites and the gene expression of the enzymes of the KYN pathway, which represent possible molecular candidates involved in depression.

\section{Materials and methods}

\subsection{Subjects}

Twelve patients (seven male (58\%) and five female (42\%) with a mean age of 45.75 years) diagnosed with chronic hepatitis C infection (HCV) were recruited from the Maastricht University Medical Center (MUMC) in the Netherlands, and assessed before and during standard treatment with pegylated IFN- $\alpha$ and ribavirin. Chronic HCV was defined as: positive for HCV-antibodies, HCV-RNA positive and elevated levels of transaminases at least once in the preceding six months.

The exclusion criteria for participation were age under 18 years and patients with a diagnosis of cancer, cerebrovascular disease, organic 
psychiatric disease and active drug abuse that may influence the research outcome. In addition, patients meeting criteria after a psychiatric interview for mental disorders such as schizophrenia, bipolar disorders, mental retardation and dementia among other neurodegenerative disorders were excluded. Lastly, analphabetism and other disorders that under the point of view of the researcher may influence the investigation were also excluded.

The study was approved by the Committee of Maastricht University Medical Center. Each subject signed a written informed consent prior to participation.

\subsection{Measurements}

A total of 12 measurements were performed during the study that lasted 48 weeks. The first time point corresponded to the baseline measurements, which were assessed 2-4 weeks before starting treatment. Next, IFN- $\alpha$ treatment started and measurements were taken every 4 weeks to monitor and measure symptom severity. At each assessment, venous blood was collected and severity of depressive symptoms was determined using the Montgomery-Asberg Depression Rating Scale (MADRS), by an independent research assistant, and the Beck Depression Inventory (BDI) by means of self-reporting.

Blood samples were collected and serum prepared according standard procedures. Samples were stored at $-80^{\circ} \mathrm{C}$. For gene expression analyses, blood was collected in PAXgene Blood RNA Tubes (PreAnalytiX GmbH, Hombrechtikon, Switzerland). RNA was extracted using the Blood RNA Kit 
(PreAnalytiX GmbH, Hombrechtikon, Switzerland) according manufacturer's instructions and stored at $-80^{\circ} \mathrm{C}$.

\subsection{Gene expression analysis}

First strand cDNA synthesis kits (Thermo scientific, Lithuania) were used to prepare cDNA. Relative gene expression analysis was performed by real time quantitative polymerase chain reaction (qPCR) using the Lightcycler 480 Real-Time PCR System (Roche Applied Science, IN, USA) and the ABI 7900 (Applied Biosystems, Singapore).

After incubation for $5 \mathrm{~min}$ at $95^{\circ} \mathrm{C}$, DNA amplification was performed over 40 cycles at $15 \mathrm{sec}$ at $95^{\circ} \mathrm{C}$, followed by a $30 \mathrm{sec}$ at both $60^{\circ} \mathrm{C}$ and $72^{\circ} \mathrm{C}$ in the lightcycler. In the $A B I$ the conditions for the reaction involved incubation for $10 \mathrm{~min}$ at $95^{\circ} \mathrm{C}$, and 40 cycles in a fixed sequence of $15 \mathrm{sec}$ at $95^{\circ} \mathrm{C}, 30 \mathrm{sec}$ at $60^{\circ} \mathrm{C}$ and $30 \mathrm{sec}$ at $72^{\circ} \mathrm{C}$. The melting curve was run at the end to confirm the identity and purity of the PCR. Primers sequences are presented in Table 1. The housekeeping genes RS27 alpha, PPIB1 and $\beta$-actin were compared in order to determine their stability. For this purpose, using a webtool (http://www.leonxie.com/referencegene.php), four different methods, e.g. Delta CT, Bestkeeper, Normfinder and Genorm were applied and determined RS27 alpha as the most stable gene. Thus, gene expression was normalized using RS27 alpha as reference gene. Glyceraldehyde 3-phosphate dehydrogenase (GAPDH) was also employed for interplate reference. Fold changes (FC) and relative gene expression values were calculated with the Pfaffl method. 
Table 1. Primer design for $\mathrm{qPCR}$

\begin{tabular}{|c|c|c|}
\hline Genes & Forward primer & Reverse primer \\
\hline IDO-1 & GCAGTAAAGGATTCTTCCTGGTC & GCATTTGCATTGCCTTGAA \\
\hline KAT-1 & TTCATGCTTAACCAGTACACCAA & CTGAGCGGGTCTATCTCCTG \\
\hline KAT-3 & GAGAAGTTTGTGCGTTTTTGC & CACTCCATGCCTTGATGATTT \\
\hline KMO & CCAGAAATACTTTCCGGATGC & AGTGAAATGAAGAGCACTTTACAGAT \\
\hline KYNase & AGAACTAGATAAGTGGGCCAAAATAG & CTCTCATCTCCTGTAATCCAAGG \\
\hline 3HAO & TGTTTGGGGACACCTATGAGA & CATTGTCACCACCGAGGAG \\
\hline QPRT & GGACAACTTCAAGCCAGAGG & GGTTGTCCAGGGTGATGC \\
\hline ActB & GTTGTCGACGACGAGCG & GCACAGAGCCTCGCCTT \\
\hline PPIB1 & GTTTGAAGTTCTCATCGGGG & AAAACAGCAAATTCCATCGTG \\
\hline RS27 alpha & GGTTAAGCTGGCTGTCCTGAA & AGAAGGGCACTCTCGACGAA \\
\hline GAPDH & CCCCGGTTTCTATAAATTGAGC & CACCTTCCCCATGGTGTCT \\
\hline
\end{tabular}

\subsection{Kynurenine metabolites analysis}

\section{$\underline{2.4 .1 \text { Instrumentation }}$}

The chromatographic system was composed of a Waters Acquity UPLC separations module connected to a Xevo TQ MS triple-quadrupole mass spectrometer, equipped with a Z-spray ESI ion source (Waters Corp., Milford, MA, U.S.). Separation was carried out using a Kinetex XB-C18, 2.6 $\mu \mathrm{m}, 2.1 \times 150 \mathrm{~mm}$ colomn (Phenomenex, Torrance, California, U.S.). 


\subsubsection{Chemicals}

Reagents for protein precipitation, derivatisation, and chromatography were purchased from Sigma-Aldrich (St. Louis, MO, U.S.), and Biosolve (Valkenswaard, NL).

\subsubsection{Standards and control samples}

TRP, KYN, 3HK, KYNA, QUIN, and 5-HIAA were purchased from SigmaAldrich (St. Louis, MO, U.S.). Standards and controls were established by adding defined volumes of the stock solutions to a human serum obtained from a blood bank. Quality control samples were generated to obtain low and high amounts of the analytes.

\subsubsection{Sample extraction}

A total sample volume of $300 \mu \mathrm{l}$ serum was used. Analytes were extracted from samples and calibrators/controls by adding $50 \mu \mathrm{l}$ of $2.0 \mathrm{M}$ urea and $50 \mu$ of internal standard solution containing KYNA-D5, PIC-D4 and TRPD5. Two precipitation steps by subsequently adding methanole/ethanol and CAN were carried out. The supernatant was separated into two portions, which were evaporated separately. One of these portions was directly reconstituted in mobile phase, while the other portion was used for derivatisation by addition of $\mathrm{HCl} /$ Butanol. After evaporation to dryness, this portion was reconstituted in mobile phase, too. 


\subsubsection{Chromatographic conditions}

$7.5 \mu \mathrm{l}$ of the reconstituted samples/calibrators/controls were loaded onto the LC-MS/MS system. 3HK and QUIN were analysed from the derivatized sample, while all other analytes were determined from the underivatized sample. A gradient method with a total duration of $7.5 \mathrm{~min}$ was used for chromatographic separation. Mobile phase A was composed of $0.1 \%$ formic acid and $0.01 \%$ HFBA in water; mobile phase B was methanol. Flow rate was set at $0.25 \mathrm{ml} / \mathrm{min}$, column temperature was set at $30.0^{\circ} \mathrm{C}$. Retention times for the analytes were between 3.1 and 6.0 $\min$.

\subsubsection{MS condition}

The Xevo TQ MS was operating in atmospheric pressure electrospray ionization (ESI) positive mode (ESI+). Ion source settings were: capillary voltage, $1.00 \mathrm{kV}$; desolvation temperatures, $650{ }^{\circ} \mathrm{C}$; source temperature, $150^{\circ} \mathrm{C}$; nitrogen was used as desolvation gas with a cone gas flow rate of $1200 \mathrm{l} / \mathrm{h}$; argon was used as collision gas at a flow rate of $0.15 \mathrm{ml} / \mathrm{min}$. The analytes and internal standards were detected using multi reaction monitoring (MRM) technique. System operation, data acquisition and data processing were controlled using MassLynx V4.1 software (Waters, Milford, USA).

\section{$\underline{2.5 \text { Statistical analysis }}$}

The statistical software program STATA (version 13.1, Statacorp, Texas, USA) was used for the analysis of the data. The metabolites TRP, KYN, 
3HK, KYNA, QUIN, and 5-HIAA, and the gene expression levels of the enzymes IDO-1, KAT-1, and KAT-3, KMO, KYNase, 3HAO and QPRT were analyzed. In addition, the ratios KYN/TRP, QUIN/KYN and KYNA/KYN were calculated. To improve normality of distributions the QUIN/KYN ratio was subjected to an inverse square root transformation, the enzymes KMO and KYNase and the metabolites 3HK, 5HIAA and KYNA were subjected to square root transformation and QPRT, IDO1 and KAT1 were log transformed.

Multilevel mixed-effects linear regression analysis was applied using the XTMIXED command of Stata. Effect sizes of explanatory variables were expressed as regression coefficients. Where appropriate, a squared term of time was added to the model ( $\beta_{1}$ : linear, $\beta_{2}$ : square), due to non-linear changes of certain variables overtime. The level of significance was set at $p<0.05$ in all cases. Analyses were corrected for age, gender and use of antidepressants, benzodiazepines and opioids during the study. Graphs were designed with Microsoft Excel.

First, effects of IFN- $\alpha$ treatment over time on the depression scores MADRS and BDI, on metabolite levels or their ratios, as well as on relative gene expression values were analyzed. Since both depression rating scales, MARDS and BDI were highly correlated (coef. 0.788, $p<0.0001$ ) and MADRS is used frequently to assess IFN- $\alpha$ induced depressive symptoms in other studies, MADRS score was regressed on the different metabolites and relative gene expression values of the enzymes to assess the association between the occurrence of depressive symptoms and metabolites or enzyme expression respectively. Last, associations 
between metabolites and expression of enzymes of the KYN pathway were examined.

\section{Results}

\subsection{Effect of IFN- $\alpha$ treatment on depression scores}

A significant change over time was observed for the MADRS $\left(\beta_{1}=2.3689\right.$, $\left.p<0.001 ; \beta_{2}=-0.1824, p<0.001\right)$ and BDI depression scores $\left(\beta_{1}=1.6639\right.$, $\left.p=0.001 ; \quad \beta_{2}=-0.1129, \quad p=0.003\right)$, during IFN- $\alpha$ treatment. While depressive symptoms steadily increased during the first 6 months of treatment, they gradually decline again in the last 4 months (see Figure 1). Post-hoc analysis indicated that MADRS scores were significantly higher from baseline at time points 3, 4, 5, 6 and 8; BDI scores were increased at time points 3, 5, 6, 7, 8, 10 and 11 .
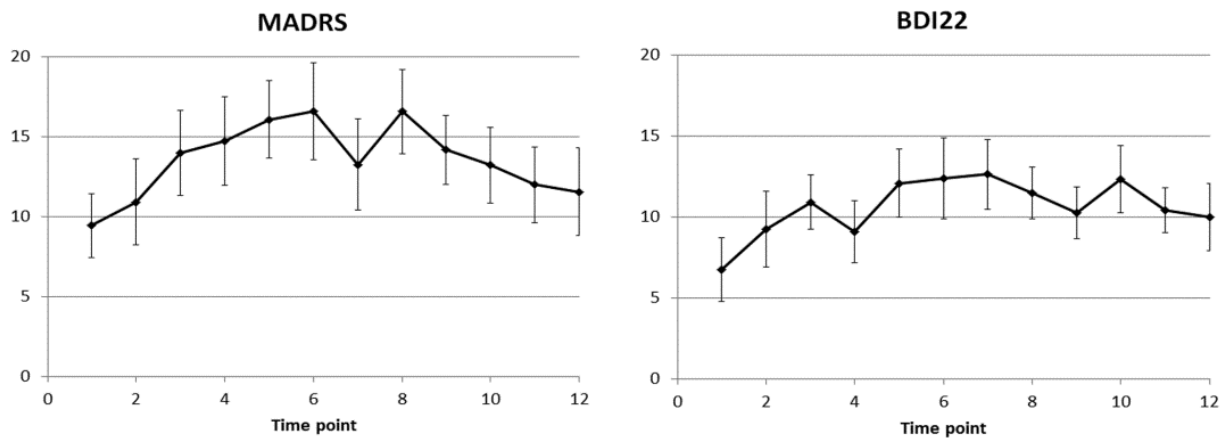

Figure 1. Change over time in MADRS and BDI scores during IFN- $\alpha$ treatment. See text for details. 


\subsection{Effect of IFN- $\alpha$ treatment on kynurenine metabolites}

A significant increase of $K Y N\left(\beta_{1}=12.4363, p=0.024\right), 3 H K\left(\beta_{1}=0.1049\right.$, $p=0.001)$ and QUIN $\left(\beta_{1}=2.8347, p=0.005\right)$ over time were observed (see Table 2 and Figure 2).

Table 2. Effect of IFN- $\alpha$ treatment over time on some metabolites of the kynurenine pathway and ratios

\begin{tabular}{lcc}
\hline Metabolites & \multicolumn{1}{c}{$\boldsymbol{\beta}$} & p-value \\
\hline TRP & -133.9359 & 0.375 \\
KYN & $\mathbf{1 2 . 4 3 6 3}$ & $\mathbf{0 . 0 2 4}$ \\
3HK & $\mathbf{0 . 1 0 4 9}$ & $\mathbf{0 . 0 0 1}$ \\
QUIN & $\mathbf{2 . 8 3 4 7}$ & $\mathbf{0 . 0 0 5}$ \\
KYNA & 0.0265 & 0.139 \\
5-HIAA & 0.0173 & 0.673 \\
\hline Ratios & $\boldsymbol{\beta}$ & $\mathbf{p}$-value \\
\hline KYN/TRP & $\mathbf{0 . 0 0 1 1}$ & $\mathbf{0 . 0 0 0}$ \\
QUIN/KYN & 0.0042 & 0.694 \\
KYNA/KYN & $\boldsymbol{\beta}_{1}=-\mathbf{0 . 0 0 1 1}$ & $\mathbf{0 . 0 0 0}$ \\
& $\boldsymbol{\beta}_{2}=\mathbf{0 . 0 0 0 1}$ & $\mathbf{0 . 0 0 1}$ \\
\hline
\end{tabular}
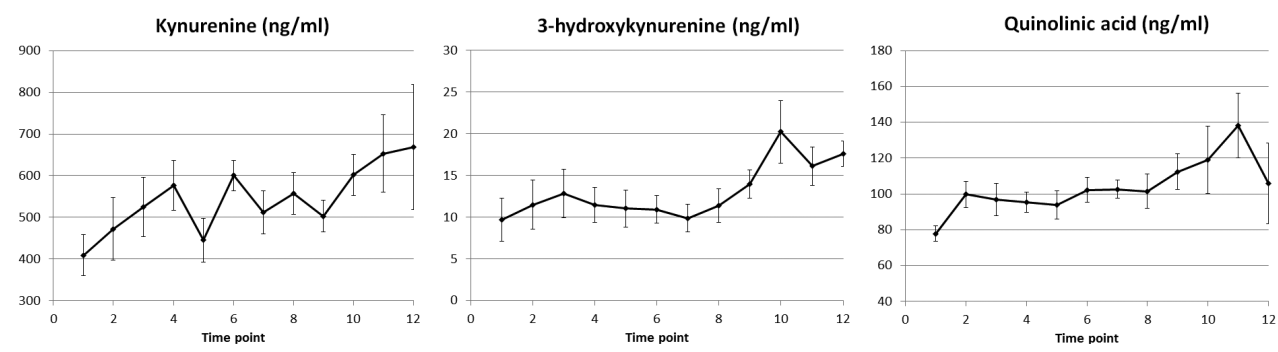

Figure 2. Change over time in serum levels of in KYN, 3HK and QUIN during IFN- $\alpha$ treatment. See text for details. 
The KYN/TRP ratio $\left(\beta_{1}=0.0011, p<0.001\right)$ significantly increased over time. The KYNA/KYN ratio also significantly changed over time $\left(\beta_{1}=-\right.$ $\left.0.0011, p<0.001 ; \beta_{2}=0.0001, p=0.001\right)$ : it gradually decreased the first 6 months and increased again thereafter (see Table 2 and Figure 3). Posthoc analyses showed that the KYNA/KYN ratio was significantly lower from baseline on time points 5, 6, 7, 8 and 9 .
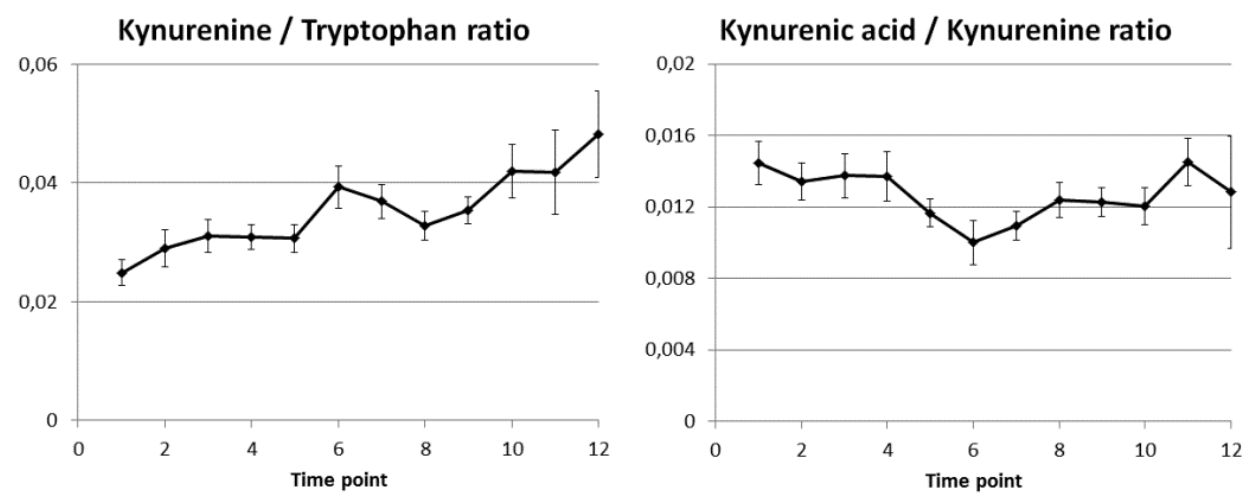

Figure 3. Change over time in the KYN/TRP and the KYNA/KYN ratios during IFN- $\alpha$ treatment. See text for details.

\subsection{Effect of IFN- $\alpha$ treatment on enzymes of the KYN pathway}

KYNase $\left(\beta_{1}=0.0057, p<0.036\right)$ was significantly increased over time during IFN- $\alpha$ treatment. In addition, a significant change over time was observed on $\mathrm{KMO}\left(\beta_{1}=0.0312, p<0.001 ; \beta_{2}=-0.0021, p<0.001\right)$ and KAT-3 $\left(\beta_{1}=\right.$ $\left.0.0140, p<0.001 ; \beta_{2}=-0.0010, p<0.001\right)$. Post hoc analysis indicated that IFN- $\alpha$ therapy increased KYNase and KMO expression in all time points, and KAT-3 in all but not 2 and 12 (see Table 3 and Figure 4). 
Table 3. Effect of IFN- $\alpha$ treatment over time on some enzymes of the kynurenine pathway

\begin{tabular}{lrc}
\hline Enzymes & $\boldsymbol{\beta}$ & $\boldsymbol{p}$-value \\
\hline IDO1 & 0.0258 & 0.159 \\
KYNase & $\mathbf{0 . 0 0 5 7}$ & $\mathbf{0 . 0 3 6}$ \\
KMO & $\boldsymbol{\beta}_{\mathbf{1}}=\mathbf{0 . 0 3 1 2}$ & $\mathbf{0 . 0 0 0}$ \\
& $\boldsymbol{\beta}_{\mathbf{2}}=\mathbf{- 0 . 0 0 2 1}$ & $\mathbf{0 . 0 0 0}$ \\
3HAO & 0.0000 & 0.901 \\
QPRT & 0.0115 & 0.615 \\
KAT1 & 0.0199 & 0.120 \\
KAT3 & $\boldsymbol{\beta}_{\mathbf{1}}=\mathbf{0 . 0 1 4 0}$ & $\mathbf{0 . 0 0 0}$ \\
& $\boldsymbol{\beta}_{\mathbf{2}}=\mathbf{- 0 . 0 0 1 0}$ & $\mathbf{0 . 0 0 0}$ \\
\hline
\end{tabular}

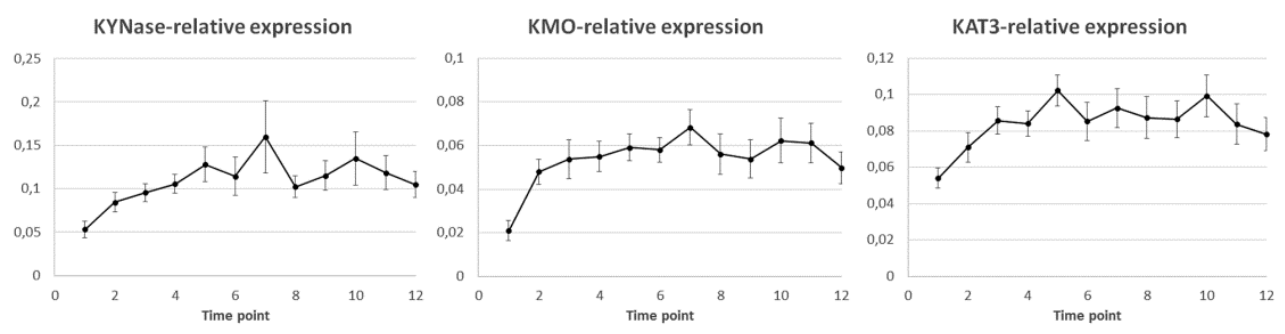

Figure 4. Change over time in KYNase, KMO and KAT-3 during IFN- $\alpha$ treatment. See text for details.

\subsection{Association between MADRS depression scores and metabolites}

KYNA was negatively associated with the MADRS score $\left(\beta_{1}=-3.4894\right.$, $p=0.029)$. In addition, a significant association was found between the ratio KYNA/KYN over time with the MADRS score $\left(\beta_{1}=-646.1161\right.$, $p=0.008)$. Associations between the rest of metabolites, e.g. KYN, QUIN, TRP, 3HK, 5-HIAA, and MADRS scores did not reach significance (see table 4). 
Table 4. Association between metabolites, ratios and symptom severity measured using MADRS

\begin{tabular}{lcc}
\hline Metabolites & $\boldsymbol{\beta}$ & $\boldsymbol{p}$-value \\
\hline TRP & -0.0003 & 0.141 \\
KYN & -0.0039 & 0.499 \\
3HK & -0.2068 & 0.863 \\
QUIN & -0.0346 & 0.268 \\
KYNA & $-\mathbf{3 . 4 8 9 4}$ & $\mathbf{0 . 0 2 9}$ \\
5-HIAA & -0.0088 & 0.991 \\
\hline Ratios & $\boldsymbol{\beta}$ & $\mathbf{p}$-value \\
\hline KYN/TRP & -58.4424 & 0.609 \\
QUIN/KYN & -0.1979 & 0.943 \\
KYNA/KYN & -646.1161 & $\mathbf{0 . 0 0 8}$ \\
\hline
\end{tabular}

\subsection{Association between MADRS depression scores and enzymes}

MADRS scores were not associated with any of the examined KYN pathway related genes (see table 5).

Table 5. Association between enzymes and symptom severity measured using MADRS

\begin{tabular}{lrc}
\hline Enzymes & \multicolumn{1}{c}{$\boldsymbol{\beta}$} & $\boldsymbol{p}$-value \\
\hline IDO1 & -0.9818 & 0.303 \\
KYNase & 4.9131 & 0.419 \\
KMO & 17.6553 & 0.147 \\
3HAO & -202.0669 & 0.737 \\
QPRT & -0.2137 & 0.765 \\
KAT1 & 1.2232 & 0.345 \\
KAT3 & 16.3008 & 0.360 \\
\hline
\end{tabular}




\subsection{Association between metabolites and enzymes}

We examined whether levels of KYN pathway metabolites could be predicted by the expression levels of the respective enzymes. Specifically, associations between the relative gene expression of the enzyme and its substrate and its product were analysed. 3HK was found positively associated with KYNase $\left(\beta_{1}=1.9877, p=0.030\right)$. No other significant associations were observed between metabolites and enzymes of the KYN pathway (see Table 6).

Table 6. Association between metabolites and enzymes of the kynurenine pathway ( $\beta_{1}$ and $p$-value)

\begin{tabular}{lccccc}
\hline & TRP & KYN & KYNA & 3HK & QUIN \\
\hline IDO1 & -119.5412 & 62.6987 & & & \\
& 0.878 & 0.219 & & & \\
KAT1 & & -43.1568 & -0.1295 & & \\
& & 0.200 & 0.162 & & \\
KAT3 & 527.8825 & 0.8131 & & \\
& & 0.332 & 0.623 & & \\
KMO & 488.5556 & & 2.9310 & \\
& & 0.134 & & 0.068 & \\
KYNase & 93.7838 & & 1.9877 & \\
& 0.625 & & 0.030 & \\
QPRT & & & & 3.1458 \\
& & & & 0.438 \\
\hline
\end{tabular}




\section{Discussion}

To the best of our knowledge, this is the most detailed study regarding longitudinal alterations in the KYN pathway in association with depressive symptoms during the course of IFN- $\alpha$ treatment. We report that, during a 12 month period of IFN- $\alpha$ treatment, depressive symptoms, as measured by the MADRS and BDI questionnaires, first steadily increase and after 6 months gradually decrease again. In addition, IFN- $\alpha$ therapy increased neurotoxic metabolites of the KYN pathway, e.g KYN, 3HK and QUIN, which was also reflected in changes of the KYN/TRP and KYNA/KYN ratios over time. Interestingly, a negative association was observed between the neuroprotective indicators (KYNA and the KYNA/KYN ratio) and the MADRS score. We also examined for the first time longitudinal changes in expression levels of genes involved in the KYN pathway. Relative expression of KMO, KYNase and KAT-3 was increased over time during IFN- $\alpha$ treatment. Finally, the enzymes KYNase and 3HK were significantly associated.

As mentioned above, one of the most effective treatments for hepatitis $C$ is IFN- $\alpha$. However, a considerable number of patients undergoing this therapy develop clinical depression (Asnis and De La Garza 2006, Papafragkakis, Rao et al. 2012). In line with our results, several studies reported higher scores for depression after IFN- $\alpha$ therapy (Raison, Dantzer et al. 2010, Shakoor, Shafqat et al. 2010, Pavlovic, Delic et al. 2011, Medeiros, Kayo et al. 2014). In these reports, the duration of IFN- $\alpha$ was differed from 12 until 48 weeks. Commonly, depression scores are measured only at the end of the treatment or at the most, on 4 time points: at baseline, 12th, 24th and 48th 
week. When comparing different time points, higher depression scores are observed mainly in the 12th and 24th week. In our study, depression scores were measured every 4 weeks during 48 weeks. Moreover, in line with the studies mentioned, we observe the highest levels of depressive symptoms after 24 (MADRS score) and 28 weeks (BDI score). We also observe a decrease in the MADRS and BDI score from week 32 on. Medeiros et al. reported a reduction in the BDI scores after 48 weeks of IFN- $\alpha$ treatment (Medeiros, Kayo et al. 2014) Interestingly, administration of several antidepressants have shown to relieve IFN- $\alpha$ induced depression (Sockalingam and Abbey 2009, Baraldi, Hepgul et al. 2012). Mainly the effects of the selective serotonin reuptake inhibitors (SSRIs) have been investigated for the treatment of IFN- $\alpha$ induced depression. For instance, sertraline showed to be efficient in resolving depression without interruption of the IFN- $\alpha$ therapy (Schramm, Lawford et al. 2000). Similar results were obtained with other SSRIs, such as paroxetine (Musselman, Lawson et al. 2001, Kraus, Schafer et al. 2002, Raison, Woolwine et al. 2007) and escitalopram (Schaefer, Sarkar et al. 2012, Qi, Zhou et al. 2013), used both as a preventive measure and as a therapy for depression. In our study, five patients received antidepressants during the study period which could explain the decline in depression scores in the second semester of the treatment. However, changes in depressive symptoms over time were not different between patients that received antidepressant treatment and those that did not, as there was no significant interaction between antidepressant treatment and time on the depression scores. This conclusion should however be taken with caution since the number of 152 
patients may be too low to find differential changes in depressive scores between both groups of patients.

A prominent role of the KYN pathway in major depression has been previously proposed (Maes, Mihaylova et al. 2007, Myint, Kim et al. 2007, Miura, Ozaki et al. 2008). Various studies reported decreased serum TRP (Maes, Verkerk et al. 1997), 5-HIAA (the main metabolite of serotonin) (Myint, Bondy et al. 2013), KYNA in plasma (Myint, Kim et al. 2007) and reduced ratio of KYNA/QUIN (Savitz, Drevets et al. 2015), but increased levels of KYN (Myint, Bondy et al. 2013) in some patients suffering from depression compared to controls. One group observed reduced TRP levels, but were unable to show changes in KYN, IDO, and other metabolites and enzymes of the KYN pathway (Hughes, Carballedo et al. 2012). Interestingly, a recent study showed a correlation between specific depressive symptoms and KYN pathway metabolites. For instance, QUIN concentration was positively correlated with the severity of guilt and the QUIN/3HK ratio was positively correlated with the severity of guilt and psychomotor agitation (Halaris, Myint et al. 2015).

It is well known that pro-inflammatory cytokines stimulate the KYN pathway (Chiarugi, Calvani et al. 2001, Guillemin, Kerr et al. 2001). Specifically, IFN- $\alpha$ and other pro-inflammatory cytokines activate IDO, the enzyme that converts TRP to KYN (Pemberton, Kerr et al. 1997, Lood, Tydén et al. 2015). Therefore, activation of the KYN pathway could be especially involved in IFN$\alpha$ induced depression. In IFN- $\alpha$ treated patients, various studies reported decreased serum levels of TRP and serotonin, and increased KYN levels (Bonaccorso, Marino et al. 2002, Wichers, Koek et al. 2004, Cozzi, Zignego et 
al. 2006). One recent study on the other hand showed increased TRP concentrations in the serum of patients treated with IFN- $\alpha$ (Oxenkrug, Turski et al. 2014), which may, although speculative, reflect an impaired transport of TRP into the brain. We did not find alterations in TRP during IFN- $\alpha$ treatment, but KYN levels steadily increased. Following the hypothesis of the activation of the KYN pathway, an increased KYN/TRP ratio, indicative of enhanced IDO activity, and a decreased KYNA/KYN ratio, indicative of augmented neurotoxic activity of the KYN cascade, are observed in IFN- $\alpha$ treated patients (Wichers, Koek et al. 2004). Moreover the KYNA/KYN ratio was negatively associated over time with MADRS score (Wichers, Koek et al. 2004). Another study reported decreased concentration of serum total TRP during IFN- $\alpha$ treatment, and increased KYN levels during treatment with IFN$\alpha$ (Comai, Cavalletto et al. 2011). The results of both studies are in agreement with those reported here. We as well observe increases in KYN and the KYN/TRP ratio, and a reduction in the KYNA/KYN ratio during IFN- $\alpha$ treatment, indicative of enhanced IDO activity and reduced production of the neuroprotective KYNA, respectively. We also found that the KYNA/KYN ratio was negatively associated over time with the MADRS score. To our knowledge, this study is the first that reports peripheral measurements of other metabolites, e.g. increased QUIN and 3HK over time in IFN- $\alpha$ treated patients. One study showed increased 3HAA levels after 8 weeks of IFN- $\alpha$ therapy. As 3HAA is the direct precursor of QUIN, they considered it as an index for neurotoxic burden of metabolites of the KYN pathway (Van Gool, Verkerk et al. 2008). To sum up, evidence points to an activation of the KYN pathway during IFN- $\alpha$ therapy, specifically of the neurotoxic arm of the 154 
pathway. Our study, with the most extensive analysis of the metabolites of the KYN pathway, further supports this theory, showing that IFN- $\alpha$ increases the neurotoxic metabolites QUIN, KYN and 3HK, and that development of depressive symptoms is negatively associated with the neuroprotective KYNA metabolite.

Several studies have used the KYN/TRP ratio as an indicator of IDO-activity and mainly reported increases during IFN- $\alpha$ therapy (Bonaccorso, Marino et al. 2002, Capuron, Neurauter et al. 2003, Wichers, Koek et al. 2004, Comai, Cavalletto et al. 2011). One study did not detect such changes (Oxenkrug, Turski et al. 2014). No studies so far have directly examined the gene expression levels of the enzymatic players in the KYN pathway during IFN- $\alpha$ treatment. We show an increase in KMO and KYNase over time during IFN$\alpha$ treatment, indicative of the increased synthesis of neurotoxic $3 \mathrm{HK}$ and 3HAA. In addition, KYNase expression significantly predicted 3HK levels. On the other hand, during IFN- $\alpha$ treatment, gene expression levels of KAT-3 were increased, but not the levels of its product KYNA. The increase of KAT3 levels may be explained because of the increase of its substrate KYN. However, the fact that no changes are observed in KYNA, suggests an activation of the neurotoxic arm of the KYN pathway over the neuroprotective one. Taken together, these data indicate that enhanced KYN pathway activity during IFN- $\alpha$ treatment is reflected in increased expression of enzymes related to the production of neurotoxic KYN metabolites. 
As we already mentioned, some patients received antidepressant treatment during the study. It may be possible that antidepressants produce some changes in metabolites or enzymes of the KYN pathway. A recent paper analyzed the effects of antidepressant treatment in several metabolites and ratios of the KYN pathway in depressed patients. They observed reductions of the neurotoxic 3HK, QUIN and a decrease in 3HK/KYN and QUIN/TRP ratios. On the other hand, the KYNA/QUIN ratio was increased (Halaris, Myint et al. 2015). In our study, all analyses were corrected for the use of antidepressants, benzodiazepines and opioids. Regression coefficients, nor significance levels, were changed when adding these variables to the models, indicating that use of antidepressants during the study period had no major effect on our analysis. Moreover, it is interesting to note that while depressive symptoms decrease during the second part of the study period, the KYN/TRP ratio - and to a lesser extend KYN, 3HK and QUIN levels steadily increase throughout. It is tempting to speculate that the neurotoxic metabolites induced by IFN- $\alpha$ initially increase depression scores, but that the subsequent relief of depressive symptoms after extended IFN- $\alpha$ exposure, whether are not mediated by antidepressants, is mediated by compensatory mechanisms that are unrelated to this part of the KYN pathway. Clearly, further examination of these processes could shed new light on the role of the KYN pathway in major depression and in IFN- $\alpha$ induced depression.

Despite the small sample size, this study shows a role for the KYN pathway in the development of depression after 48 weeks of treatment with IFN- $\alpha$, as indicated by the increasing KYN/TRP ratio over time. Specifically, we 156 
report enhanced levels over time of the considered neurotoxic metabolites of the KYN pathway, as well as increased concentrations of the enzymes regulating this arm of the pathway. In addition, the negative association observed between the ratio KYNA/KYN and MADRS scores, indicates the importance of the neuroprotective KYNA in the development of depressive symptoms. Evidently, it would be of great value to further explore the exact role of the KYN pathway in IFN- $\alpha$ induced depression in order to understand the precise mechanisms involved. Further research should also address how antidepressants, as a preventive measure or therapy, could affect the above mentioned metabolites and enzymes.

\section{Acknowledgements}

The study was supported by the Commission of European Communities $7^{\text {th }}$ Framework Programme collaborative project "MOODINFLAME" (Grant No. 22963).

The authors would like to thank Barbie Machiels for their guidance and help in the performance of the experiments. 


\section{References}

Asnis, G. M. and R. De La Garza, 2nd (2006). "Interferon-induced depression in chronic hepatitis C: a review of its prevalence, risk factors, biology, and treatment approaches." J Clin Gastroenterol 40(4): 322-335.

Baraldi, S., N. Hepgul, V. Mondelli and C. M. Pariante (2012). "Symptomatic treatment of interferon-alpha-induced depression in hepatitis C: a systematic review." J Clin Psychopharmacol 32(4): 531-543.

Bender, D. A. and G. M. McCreanor (1985). "Kynurenine hydroxylase: a potential rate-limiting enzyme in tryptophan metabolism." Biochem Soc Trans 13(2): 441-443.

Bonaccorso, S., V. Marino, A. Puzella, M. Pasquini, M. Biondi, M. Artini, C. Almerighi, R. Verkerk, H. Meltzer and M. Maes (2002). "Increased depressive ratings in patients with hepatitis $C$ receiving interferon-alpha-based immunotherapy are related to interferon-alpha-induced changes in the serotonergic system." J Clin Psychopharmacol 22(1): 86-90.

Capuron, L. and A. H. Miller (2011). "Immune system to brain signaling: neuropsychopharmacological implications." Pharmacol Ther 130(2): 226238.

Capuron, L., G. Neurauter, D. L. Musselman, D. H. Lawson, C. B. Nemeroff, D. Fuchs and A. H. Miller (2003). "Interferon-alpha-induced changes in tryptophan metabolism: relationship to depression and paroxetine treatment." Biological Psychiatry 54(9): 906-914.

Chiarugi, A., M. Calvani, E. Meli, E. Traggiai and F. Moroni (2001). "Synthesis and release of neurotoxic kynurenine metabolites by human monocyte-derived macrophages." Journal of Neuroimmunology 120(1-2): 190-198.

Christmas, D. M., J. Potokar and S. J. Davies (2011). "A biological pathway linking inflammation and depression: activation of indoleamine 2,3-dioxygenase." Neuropsychiatr Dis Treat 7: 431-439.

Comai, S., L. Cavalletto, L. Chemello, E. Bernardinello, E. Ragazzi, C. V. L. Costa and A. Bertazzo (2011). "Effects of PEG-interferon alpha plus ribavirin on tryptophan metabolism in patients with chronic hepatitis C." Pharmacological Research 63(1): 85-92.

Cozzi, A., A. L. Zignego, R. Carpendo, T. Biagiotti, A. Aldinucci, M. Monti, C. Giannini, M. Rosselli, G. Laffi and F. Moroni (2006). "Low serum tryptophan levels, reduced macrophage IDO activity and high frequency of psychopathology in HCV patients." J Viral Hepat 13(6): 402-408.

Debien, C., M. B. De Chouly De Lenclave, P. Foutrein and D. Bailly (2001). "[Alphainterferon and mental disorders]." Encephale 27(4): 308-317.

Foster, G. (2010). "Pegylated Interferons for the Treatment of Chronic Hepatitis C." Drugs 70(2): 147-165. 
Garthwaite, G. and J. Garthwaite (1987). "Quinolinate mimics neurotoxic actions of $\mathrm{N}$-methyl-D-aspartate in rat cerebellar slices." Neurosci Lett 79(1-2): 35-39.

Guillemin, G. J., S. J. Kerr, L. A. Pemberton, D. G. Smith, G. A. Smythe, P. J. Armati and B. J. Brew (2001). "IFN-beta1b induces kynurenine pathway metabolism in human macrophages: potential implications for multiple sclerosis treatment." J Interferon Cytokine Res 21(12): 1097-1101.

Halaris, A., A. M. Myint, V. Savant, E. Meresh, E. Lim, G. Guillemin, D. Hoppensteadt, J. Fareed and J. Sinacore (2015). "Does escitalopram reduce neurotoxicity in major depression?" J Psychiatr Res 66-67: 118-126.

Haroon, E., C. L. Raison and A. H. Miller (2012). "Psychoneuroimmunology meets neuropsychopharmacology: translational implications of the impact of inflammation on behavior." Neuropsychopharmacology 37(1): 137-162.

Heyes, M. P., C. L. Achim, C. A. Wiley, E. O. Major, K. Saito and S. P. Markey (1996). "Human microglia convert I-tryptophan into the neurotoxin quinolinic acid." Biochemical Journal 320(Pt 2): 595-597.

Hoyo-Becerra, C., J. F. Schlaak and D. M. Hermann (2014). "Insights from interferonalpha-related depression for the pathogenesis of depression associated with inflammation." Brain Behav Immun 42: 222-231.

Hughes, M. M., A. Carballedo, D. M. McLoughlin, F. Amico, A. Harkin, T. Frodl and T. J. Connor (2012). "Tryptophan depletion in depressed patients occurs independent of kynurenine pathway activation." Brain, Behavior, and Immunity 26(6): 979-987.

Khaspekov, L., E. Kida, I. Victorov and M. J. Mossakowski (1989). "Neurotoxic effect induced by quinolinic acid in dissociated cell culture of mouse hippocampus." J Neurosci Res 22(2): 150-157.

Kraus, M. R., A. Schafer, H. Faller, H. Csef and M. Scheurlen (2002). "Paroxetine for the treatment of interferon-alpha-induced depression in chronic hepatitis C." Aliment Pharmacol Ther 16(6): 1091-1099.

Lood, C., H. Tydén, B. Gullstrand, C. Klint, C. Wenglén, C. T. Nielsen, N. H. H. Heegaard, A. Jönsen, R. Kahn and A. A. Bengtsson (2015). "Type I Interferon-Mediated Skewing of the Serotonin Synthesis Is Associated with Severe Disease in Systemic Lupus Erythematosus." PLOS ONE 10(4): e0125109.

Maes, M., I. Mihaylova, M. D. Ruyter, M. Kubera and E. Bosmans (2007). "The immune effects of TRYCATs (tryptophan catabolites along the IDO pathway): relevance for depression - and other conditions characterized by tryptophan depletion induced by inflammation." Neuro Endocrinol Lett 28(6): 826-831.

Maes, M., R. Verkerk, E. Vandoolaeghe, F. Van Hunsel, H. Neels, A. Wauters, P. Demedts and S. Scharpe (1997). "Serotonin-immune interactions in major 
depression: lower serum tryptophan as a marker of an immuneinflammatory response." Eur Arch Psychiatry Clin Neurosci 247(3): 154-161. Medeiros, L. P. J. d., M. Kayo, R. B. V. Medeiros, M. B. C. Lima and C. E. B. M. Mello (2014). "Interferon-induced depression in patients with hepatitis $C$ : an epidemiologic study." Revista da Associação Médica Brasileira 60: 35-39.

Miura, H., N. Ozaki, M. Sawada, K. Isobe, T. Ohta and T. Nagatsu (2008). "A link between stress and depression: shifts in the balance between the kynurenine and serotonin pathways of tryptophan metabolism and the etiology and pathophysiology of depression." Stress 11(3): 198-209.

Musselman, D. L., D. H. Lawson, J. F. Gumnick, A. K. Manatunga, S. Penna, R. S. Goodkin, K. Greiner, C. B. Nemeroff and A. H. Miller (2001). "Paroxetine for the prevention of depression induced by high-dose interferon alfa." $\mathrm{N} \mathrm{Engl}$ J Med 344(13): 961-966.

Myint, A.-M., Y. K. Kim, R. Verkerk, S. Scharpé, H. Steinbusch and B. Leonard (2007). "Kynurenine pathway in major depression: Evidence of impaired neuroprotection." Journal of Affective Disorders 98(1-2): 143-151.

Myint, A. M. (2012). "Kynurenines: from the perspective of major psychiatric disorders." FEBS Journal 279(8): 1375-1385.

Myint, A. M., B. Bondy, T. C. Baghai, D. Eser, C. Nothdurfter, C. Schüle, P. Zill, N. Müller, R. Rupprecht and M. J. Schwarz (2013). "Tryptophan metabolism and immunogenetics in major depression: A role for interferon- $\gamma$ gene." Brain, Behavior, and Immunity 31(0): 128-133.

Oxenkrug, G., W. Turski, W. Zgrajka, J. Weinstock, R. Ruthazer and P. Summergrad (2014). "Disturbances of Tryptophan Metabolism and Risk of Depression in HCV Patients Treated with IFN-Alpha." J Infect Dis Ther 2(2).

Papafragkakis, H., M. Rao, M. Moehlen, S. Dhillon and P. Martin (2012). "Depression and pegylated interferon-based hepatitis C treatment." International Journal of Interferon, Cytokine and Mediator Research 4: 25-35.

Pavlovic, Z., D. Delic, N. P. Maric, O. Vukovic and M. Jasovic-Gasic (2011). "Depressive symptoms in patients with hepatitis $C$ treated with pegylated interferon alpha therapy: a 24-week prospective study." Psychiatr Danub 23(4): 370-377.

Pemberton, L. A., S. J. Kerr, G. Smythe and B. J. Brew (1997). "Quinolinic acid production by macrophages stimulated with IFN-gamma, TNF-alpha, and IFN-alpha." J Interferon Cytokine Res 17(10): 589-595.

Qi, M., B. Zhou, M. Su, J. Pan and H. Zhang (2013). "[Escitalopram for intervention of psychiatric adverse events during peginterferon-alfa-2a and ribavirin treatment for chronic hepatitis C]." Nan Fang Yi Ke Da Xue Xue Bao 33(7): 1012-1016. 
Raison, C. L., R. Dantzer, K. W. Kelley, M. A. Lawson, B. J. Woolwine, G. Vogt, J. R. Spivey, K. Saito and A. H. Miller (2010). "CSF concentrations of brain tryptophan and kynurenines during immune stimulation with IFN-[alpha]: relationship to CNS immune responses and depression." Mol Psychiatry 15(4): 393-403.

Raison, C. L., B. J. Woolwine, M. F. Demetrashvili, A. S. Borisov, R. Weinreib, J. P. Staab, J. M. Zajecka, C. J. Bruno, M. A. Henderson, J. F. Reinus, D. L. Evans, G. M. Asnis and A. H. Miller (2007). "Paroxetine for prevention of depressive symptoms induced by interferon-alpha and ribavirin for hepatitis C." Aliment Pharmacol Ther 25(10): 1163-1174.

Savitz, J., W. C. Drevets, B. E. Wurfel, B. N. Ford, P. S. F. Bellgowan, T. A. Victor, J. Bodurka, T. K. Teague and R. Dantzer (2015). "Reduction of kynurenic acid to quinolinic acid ratio in both the depressed and remitted phases of major depressive disorder." Brain, Behavior, and Immunity 46(0): 55-59.

Schaefer, M., A. Hinzpeter, A. Mohmand, G. Janssen, M. Pich, M. Schwaiger, R. Sarkar, A. Friebe, A. Heinz, M. Kluschke, M. Ziemer, J. Gutsche, V. Weich, J. Halangk and T. Berg (2007). "Hepatitis C treatment in "difficult-to-treat" psychiatric patients with pegylated interferon-alpha and ribavirin: Response and psychiatric side effects." Hepatology 46(4): 991-998.

Schaefer, M., R. Sarkar, V. Knop, S. Effenberger, A. Friebe, L. Heinze, U. Spengler, T. Schlaepfer, J. Reimer, P. Buggisch, J. Ockenga, R. Link, M. Rentrop, H. Weidenbach, G. Fromm, K. Lieb, T. F. Baumert, A. Heinz, T. Discher, K. Neumann, S. Zeuzem and T. Berg (2012). "Escitalopram for the prevention of peginterferon-alpha2a-associated depression in hepatitis C virusinfected patients without previous psychiatric disease: a randomized trial." Ann Intern Med 157(2): 94-103.

Schramm, T. M., B. R. Lawford, G. A. Macdonald and W. G. Cooksley (2000). "Sertraline treatment of interferon-alfa-induced depressive disorder." Med J Aust 173(7): 359-361.

Shakoor, A., F. Shafqat, T. Mehmud, M. Akram, S. Riaz, Z. Iqbal and A. A. Khan (2010). "Frequency of depression and somatic symptoms in patients on interferon alpha/ribavirin for chronic hepatitis C." J Ayub Med Coll Abbottabad 22(4): 6-9.

Sockalingam, S. and S. E. Abbey (2009). "Managing depression during hepatitis C treatment." Can J Psychiatry 54(9): 614-625.

Van Gool, A. R., R. Verkerk, D. Fekkes, M. Bannink, S. Sleijfer, W. H. Kruit, B. van der Holt, S. Scharpe, A. M. Eggermont, G. Stoter and M. W. Hengeveld (2008). "Neurotoxic and neuroprotective metabolites of kynurenine in patients with renal cell carcinoma treated with interferon-alpha: course and 
relationship with psychiatric status." Psychiatry Clin Neurosci 62(5): 597602.

Wichers, M. C., G. Kenis, G. H. Koek, G. Robaeys, N. A. Nicolson and M. Maes (2007). "Interferon-alpha-induced depressive symptoms are related to changes in the cytokine network but not to cortisol." J Psychosom Res 62 (2): 207-214. Wichers, M. C., G. H. Koek, G. Robaeys, R. Verkerk, S. Scharpe and M. Maes (2004). "IDO and interferon-[alpha]-induced depressive symptoms: a shift in hypothesis from tryptophan depletion to neurotoxicity." Mol Psychiatry 10(6): 538-544. 


\title{
Chapter 7
}

General discussion

\author{
Yara Pujol López
}




\section{General discussion}

Increasing evidence shows that infection during the pregnancy affects brain development in the offspring, increasing their susceptibility to develop psychopathology later in life. The underlying mechanisms mediating this relation still remain to be elucidated though. Several mediators have been proposed to be involved, such as epigenetic mechanisms and the kynurenine metabolism. The possible role of these factors is examined in the different chapters of this thesis.

Chapter 2 represents a review of the literature, addressing the association between prenatal maternal infection and the development of psychiatric disorders in the offspring in later life, in particular depression. Several hypotheses on the pathogenesis of depression and the role of possible mediators, such as epigenetic programming, are being discussed. In addition, we addressed the function of the immune system during normal pregnancy and the changes observed after infection during gestation. Moreover, the kynurenine pathway and downstream neurotoxic and neuroprotective metabolites are discussed. In addition, this chapter highlights the consequences of pregnancy stress on the immune system, the effects of which are strikingly similar to those of prenatal maternal infection. As depression is more likely to occur in women, gender differences are also evaluated. Lastly, an overview of the current available treatment options is given.

While reviewing the literature, we could notice a lack of direct evidence linking prenatal maternal infection and the development of depressionrelated phenotypes in the offspring, both in animal and human studies. In 
animals, exposure to prenatal infection has been mostly studied in relation to the development of schizophrenia (Zuckerman, Rehavi et al. 2003, Meyer, Feldon et al. 2005, Zuckerman and Weiner 2005, Piontkewitz, Assaf et al. 2009, Bitanihirwe, Peleg-Raibstein et al. 2010, Howland, Cazakoff et al. 2012, Wischhof, Irrsack et al. 2015). In line with this, several epidemiological studies showed that infection during pregnancy was associated with an increased risk of schizophrenia in the offspring (Brown, Begg et al. 2004, Babulas, Factor-Litvak et al. 2006, Mortensen, Nørgaard-Pedersen et al. 2007, Sørensen, Mortensen et al. 2009). Interestingly, we were not able to find similar studies related to the development of depression. Moreover, only few animal studies examined the consequences of prenatal maternal infection on depression-related behavior (Enayati, Solati et al. 2012, Khan, Fernando et al. 2014). Clearly, it would be of great interest to further explore the possible link between prenatal infection and the development of depression in the offspring employing both animal models and epidemiological studies. Thus, prospective human studies together with more epidemiological studies exploring the above mentioned link would be of great value in the field.

In $\underline{\text { Chapter } 3}$, we examined how a subcutaneous injection of LPS at GD 17 influenced gestational length and offspring mortality. It is known that the effects of infection exerted on the dam and the offspring differ depending on the timing, dose and route of administration (Mijovic, Zakar et al. 2002, Nakamura, Mori et al. 2003, Renaud, Cotechini et al. 2011). As such, it is challenging to find the right combination of dose, route and timing of injection that will produce the effects that the investigator is aiming for. 
Moreover, it is remarkable that pregnant rodent dams are commonly subjected to an intraperitoneal injection (Buhimschi, Buhimschi et al. 2003, Xu, Chen et al. 2006, Salminen, Paananen et al. 2008), as a misplaced injection could be dangerous for both the mother and the foetus(es). Given the lack of evidence using subcutaneous injections of LPS in pregnant mice, we decided to test the effects of subcutaneous LPS administration using a dose of $2 \mathrm{mg} / \mathrm{kg}$ and $0.5 \mathrm{mg} / \mathrm{kg}$. We found that gestational length was markedly reduced and neonatal mortality was dramatically increased after using the higher dose of LPS. In addition, litter size tended to be reduced after the administration of a low dose of LPS. More specifically, $2 \mathrm{mg} / \mathrm{kg}$ of LPS injected subcutaneously resulted in an abrupt premature delivery, concomitant with an elevated degree of neonatal mortality, while injection of $0.5 \mathrm{mg} / \mathrm{kg}$ LPS caused an increase in prenatal mortality that could be explained by reabsorption of foetuses. Altogether, we suggest that subcutaneous injections may be more suitable than intraperitoneal injections when dealing with pregnant dams. Following the studies performed here, it would be of clear added value to examine the exact effects of maternal LPS exposure in pups when using different doses, routes and time points of administration. This will help to choose the suitable combination when planning future experiments.

In Chapter 4 we investigated brain quinolinic acid (QUIN) expression in naïve adult mice. Altered QUIN expression has been reported in patients suffering from psychiatric disorders (Németh, Toldi et al. 2006, Gulaj, Pawlak et al. 2010, Raison, Dantzer et al. 2010), while its neurotoxic properties are well known (Nemeth, Toldi et al. 2005, Maes, Yirmyia et al. 2009). Although 
several studies had already examined QUIN expression in different disease states and models (Baratte, Molinari et al. 1998, Guillemin, Brew et al. 2005, Guillemin, Smythe et al. 2005, Chung, Leung et al. 2009), knowledge on its distribution and pattern of staining in naïve mice was still lacking. We showed that QUIN expression was particularly pronounced in the cingulate cortex (CC) and the thalamic reticular nucleus (TRN) and to a similar degree in males and females. In the $\mathrm{CC}$, we could observe clearly delineated individual cells, whereas in the TRN the staining pattern was much more diffuse. Furthermore, we found that the number of QUIN-positive cells observed in the CC was correlated to the degree of QUIN-immunoreactivity in the TRN. Interestingly, these two brain areas are functionally and anatomically connected and it is known that the TRN receives projections from the cortex (Pinault 2004, Çavdar, Onat et al. 2008). In addition, in males only, QUIN-immunoreactivity in the TRN was strongly correlated with locomotion. This association suggests QUIN as a possible mediator in regulating motor behavior at the level of the TRN. Interestingly, the TRN has been reported to be involved in the attention deficit hyperactivity disorder (ADHD), specifically in the modulation of arousal (di Michele, Prichep et al. 2005, Rowe, Robinson et al. 2005), and a recent paper showed a positive correlation between the baseline QUIN/3HK ratio and the severity of psychomotor agitation in patients with major depression (Halaris, Myint et al. 2015). It would be interesting in future studies to perform behavioural tests more closely related to primary functions of the CC and the TRN, such as memory and navigation. Furthermore, it would be of value to examine the exact nature of the cells displaying QUIN-immunoreactivity, e.g. by using 
double-labeling immunofluorescence. Moreover, our findings, in line with recent evidence from other studies suggest that the role of QUIN in locomotion should be further investigated as well as the role of QUIN in the CC and the TRN in models of stress and/or inflammation linked to depression. Finally, the evaluation of other metabolites such as kynurenic acid, may shed new light on the field.

In $\underline{\text { Chapter } 5}$, we evaluated changes in global enzymatic histone deacetylase (HDAC) and DNA methylation (DNMT) activity, after prenatal maternal exposure to Poly I:C at GD17. Poly I:C has been commonly used to mimic a viral infection (Doukas, Cutler et al. 1994, Snell, Chernyshev et al. 1997). Infection and consequent inflammation during gestation have been associated with an increased risk to develop psychiatric disorders in the offspring in later life (Fatemi, Reutiman et al. 2008, Zhou 2012). In this study, we investigated the possible role of epigenetic changes, i.e. in HDAC and DNMT activity, as potential mechanisms underlying the association between infection during pregnancy and offspring psychopathology. Results indicated a sex-specific effect of prenatal maternal Poly I:C exposure on brain HDAC activity, with an increased activity observed in female - but not male - Poly $\mathrm{I}: \mathrm{C}$ offspring when compared to controls. Subsequently, we speculated which HDAC isoform may be mediating the increased activity observed. In this respect, western blot experiments could, however, not identify a specific HDAC. We examined HDAC1, HDAC2, HDAC3, HDAC4 and HDAC6, because of the evidence showing their association with mental (dys)function (McQuown, Barrett et al. 2011, Bahari-Javan, Maddalena et al. 2012, Fukada, Hanai et al. 2012, Morris, Mahgoub et al. 2013, Sarkar, Chachra et al. 2014). 
Furthermore, we initially included HDAC5, because of its role in inflammation (Cantley and Haynes 2013), and that reduced levels of HDAC5 have been associated to the antidepressant effects of imipramine in mice models (Tsankova, Berton et al. 2006). However, we were not able to identify a specific band for HDAC5 after using various antibodies in several organ tissues. Possibly, other HDAC molecules than those assessed may be involved in mediating the effects of prenatal maternal Poly I:C exposure on global HDAC activity. In addition, a higher enzymatic activity does not necessarily imply an increase in the level of the associated protein(s). Clearly, this issue awaits further research. Of note, as mentioned in Chapter 5, it would of additional interest to examine the brain region- and cell-typespecific epigenetic effects of prenatal maternal Poly I:C exposure in order to unravel the exact role of epigenetic programming in mediating the effects of prenatal maternal infection. In addition to differences in the degree of histone acetylation, in part mediated via altered HDAC activity, it would be of great value to identify other epigenetic players that mediate the effects of prenatal maternal infection on offspring brain development.

In Chapter 6, we explored the role of the kynurenine pathway in relation to the development of depressive symptoms in patients treated with interferon-alpha (IFN- $\alpha$ ). Patients diagnosed with hepatitis $\mathrm{C}$ are commonly treated with IFN- $\alpha$, and a considerable percentage has been reported to develop depression (Papafragkakis, Rao et al. 2012). It is well-known, that cytokines, such as IFN- $\alpha$, regulate diverse enzymes of the kynurenine (KYN) pathway (Myint 2012), whose metabolites have been found altered in depressed patients (Bonaccorso, Marino et al. 2002, Capuron, Neurauter et 
al. 2003, Wichers, Koek et al. 2005, Cozzi, Zignego et al. 2006, Comai, Cavalletto et al. 2011, Hughes, Carballedo et al. 2012, Myint, Bondy et al. 2013, Oxenkrug, Turski et al. 2014), albeit with discordant results. Thus, we decided to analyse gene expression levels of several enzymes as well as concentrations of metabolites of the KYN pathway in IFN- $\alpha$ treated patients. We observed significant changes during the 48 weeks of IFN- $\alpha$ treatment in the Montgomery-Asberg Depression Rating Scale (MADRS) and the Beck Depression Inventory (BDI), Kynurenine (KYN), 3-Hydroxykynurenine (3HK), Quinolinic acid (QUIN), the ratio Kynurenine/Tryptophan and the Kynurenic Acid/Kynurenine ratio. In addition, kynureninase, kynurenine-3monooxygenase and kynurenine aminotransferase 3 enzymes were changed over time during the IFN- $\alpha$ treatment. Next, we observed that only KYNA and the ratio KYNA/KYN were negatively associated with MADRS scores over time, whereas no association was found between gene expression levels of the studied enzymes and MADRS scores. Finally, we analysed possible associations between the enzymes and their precursors and products. Only $3 \mathrm{HK}$ was positively associated with KYNase. These results suggest that the KYN pathway, and specifically its neurotoxic metabolites, e.g. 3HK, QUIN and the enzymes that regulate the neurotoxic arm of the pathway, have a role in IFN- $\alpha$ induced depression. On the other hand, the neuroprotective arm, as represented by KYNA levels, is negatively associated with depressive symptoms. Overall, IFN- $\alpha$ shifts the neurotoxic/neuroprotective balance of the KYN pathway metabolites to the neurotoxic arm resulting in the precipitation of depressive symptoms. Future investigations should further examine the KYN pathway in order to better understand the exact 
mechanisms involved in IFN- $\alpha$ induced depression and in general major depression. It would be also of interest to assess whether or not the therapeutic or prophylactic use of antidepressants may influence metabolites and enzymes of the KYN pathway.

\section{Methodological considerations}

All in all, the work associated with this thesis yielded numerous valuable insights regarding the design and execution of animal work on prenatal maternal infection, the most important of which concerns controlling for litter effects. Pups from a single litter show a limited degree of genetic, epigenetic and environmental variation (Meyer, Feldon et al. 2009). Thus, when assessing the effects of prenatal events on measures in the offspring, we may confound treatment effects with so-called litter effects (Chapman and Stern 1978, Lazic and Essioux 2013). Principally, in studies like these, the dams/litter should be considered as the experimental/statistical unit, and not the pup(s). A way to avoid these litter effects is including only 1-2 pup(s) per sex from each litter when assessing a specific experimental variable (Hass, Ladefoged et al. 2001, Upton and Sullivan 2010). In Chapters 3 and 4, pregnant dams were exposed to maternal infection. Mice were injected s.c. or i.p. with LPS, Poly I:C or PBS, respectively, at GD17. After weaning, offspring was separated by sex and treatment, whereas litter effects were not controlled for. Unfortunately, at a later stage, it was impossible to trace back which pups belonged to the same litter. Consequently, the behavioural measurements assessed in these animals could not be used to directly compare offspring exposed to prenatal maternal LPS or Poly I:C and controls. 


\section{Concluding remarks}

Psychiatric disorders are leading cause of disability worldwide. More specifically, major depression affects more than 100 million people per year. Despite the repercussion of this disorder on our personal life, economy and health, a reliable biomarker to diagnose depression has not been developed yet, while treatment efficacy is relatively low. In this thesis, we tried to assess a variety of factors, such as epigenetic processes as well as metabolites and enzymes of the kynurenine pathway, in the context of inflammation, in order to identify potential biomarkers and an effective treatment for depression. We observed that LPS administration during pregnancy in the mouse had a profound impact upon gestational length, litter size and perinatal mortality in the offspring, even at a relatively low dose. In the long run, female offspring of dams exposed to Poly I:C during pregnancy showed increased global HDAC activity when compared to control offspring. Regarding the role of the kynurenine pathway, we observed QUIN-immunoreactivity in two brain areas in naïve mouse, the CC and the TRN. Interestingly, we observed an association between QUINimmunoreactivity in the TRN and locomotion, which suggests that QUIN is involved in the regulation of motor activity. Furthermore, the role of kynurenine pathway was assessed in IFN- $\alpha$ treated patients, indicating the importance of the neurotoxic metabolites and related enzymes of the pathway, and the association between depressive symptoms and reduced neuroprotective metabolites.

We hypothesized that alterations in the kynurenine pathway are causally involved in mediating changes in inflammation-induced depression-related 
phenotypes. Moreover, we postulated epigenetic changes as mechanisms underlying the association between prenatal inflammation and adult psychopathology. Altogether, we showed that prenatal maternal inflammation mediated changes in epigenetic marks, while exposure to cytokines in adulthood affected the kynurenine metabolism in relation to depression. A better understanding of the processes underlying the negative consequences of prenatal maternal inflammation as well as those induced by exposure to cytokines in adulthood will help for an early diagnose and better targeted treatment.

\section{Disclosure:}

Yara Pujol Lopez and Aye-Mu Myint have been partially employed by ApDia from 15-09-2012 to 15-12-2012, and 21-05-2009 to 31-03-2015, respectively. 


\section{References}

Babulas, V., P. Factor-Litvak, R. Goetz, C. A. Schaefer and A. S. Brown (2006). "Prenatal exposure to maternal genital and reproductive infections and adult schizophrenia." Am J Psychiatry 163(5): 927-929.

Bahari-Javan, S., A. Maddalena, C. Kerimoglu, J. Wittnam, T. Held, M. Bahr, S. Burkhardt, I. Delalle, S. Kugler, A. Fischer and F. Sananbenesi (2012). "HDAC1 regulates fear extinction in mice." J Neurosci 32(15): 5062-5073.

Baratte, S., A. Molinari, O. Veneroni, C. Speciale, L. Benatti and P. Salvati (1998). "Temporal and spatial changes of quinolinic acid immunoreactivity in the gerbil hippocampus following transient cerebral ischemia." Molecular brain research 59(1): 50-57.

Bitanihirwe, B. K., D. Peleg-Raibstein, F. Mouttet, J. Feldon and U. Meyer (2010). "Late prenatal immune activation in mice leads to behavioral and neurochemical abnormalities relevant to the negative symptoms of schizophrenia." Neuropsychopharmacology 35(12): 2462-2478.

Bonaccorso, S., V. Marino, A. Puzella, M. Pasquini, M. Biondi, M. Artini, C. Almerighi, R. Verkerk, H. Meltzer and M. Maes (2002). "Increased depressive ratings in patients with hepatitis $C$ receiving interferon-alpha-based immunotherapy are related to interferon-alpha-induced changes in the serotonergic system." J Clin Psychopharmacol 22(1): 86-90.

Brown, A. S., M. D. Begg, S. Gravenstein and et al. (2004). "SErologic evidence of prenatal influenza in the etiology of schizophrenia." Archives of General Psychiatry 61(8): 774-780.

Buhimschi, I. A., C. S. Buhimschi and C. P. Weiner (2003). "Protective effect of Nacetylcysteine against fetal death and preterm labor induced by maternal inflammation." American Journal of Obstetrics and Gynecology 188(1): 203208.

Cantley, M. and D. Haynes (2013). "Epigenetic regulation of inflammation: progressing from broad acting histone deacetylase (HDAC) inhibitors to targeting specific HDACs." Inflammopharmacology 21(4): 301-307.

Capuron, L., G. Neurauter, D. L. Musselman, D. H. Lawson, C. B. Nemeroff, D. Fuchs and A. H. Miller (2003). "Interferon-alpha-induced changes in tryptophan metabolism. relationship to depression and paroxetine treatment." Biol Psychiatry 54(9): 906-914.

Çavdar, S., F. Y. Onat, Y. Ö. Çakmak, H. R. Yananli, M. Gülçebi and R. Aker (2008). "The pathways connecting the hippocampal formation, the thalamic reuniens nucleus and the thalamic reticular nucleus in the rat." Journal of Anatomy 212(3): 249-256.

Chapman, R. H. and J. M. Stern (1978). "Maternal stress and pituitary-adrenal manipulations during pregnancy in rats: Effects on morphology and sexual 
behavior of male offspring." Journal of comparative and physiological psychology 92(6): 1074.

Chung, R. S., Y. K. Leung, C. W. Butler, Y. Chen, E. D. Eaton, M. W. Pankhurst, A. K. West and G. J. Guillemin (2009). "Metallothionein treatment attenuates microglial activation and expression of neurotoxic quinolinic acid following traumatic brain injury." Neurotox Res 15(4): 381-389.

Comai, S., L. Cavalletto, L. Chemello, E. Bernardinello, E. Ragazzi, C. V. L. Costa and A. Bertazzo (2011). "Effects of PEG-interferon alpha plus ribavirin on tryptophan metabolism in patients with chronic hepatitis C." Pharmacological Research 63(1): 85-92.

Cozzi, A., A. L. Zignego, R. Carpendo, T. Biagiotti, A. Aldinucci, M. Monti, C. Giannini, M. Rosselli, G. Laffi and F. Moroni (2006). "Low serum tryptophan levels, reduced macrophage IDO activity and high frequency of psychopathology in HCV patients." J Viral Hepat 13(6): 402-408.

di Michele, F., L. Prichep, E. R. John and R. J. Chabot (2005). "The neurophysiology of attention-deficit/hyperactivity disorder." Int J Psychophysiol 58(1): 8193.

Doukas, J., A. H. Cutler and J. P. Mordes (1994). "Polyinosinic:polycytidylic acid is a potent activator of endothelial cells." Am J Pathol 145(1): 137-147.

Enayati, M., J. Solati, M.-H. Hosseini, H.-R. Shahi, G. Saki and A.-A. Salari (2012). "Maternal infection during late pregnancy increases anxiety- and depression-like behaviors with increasing age in male offspring." Brain Research Bulletin 87(2-3): 295-302.

Fatemi, S. H., T. J. Reutiman, T. D. Folsom, H. Huang, K. Oishi, S. Mori, D. F. Smee, D. A. Pearce, C. Winter, R. Sohr and G. Juckel (2008). "Maternal infection leads to abnormal gene regulation and brain atrophy in mouse offspring: implications for genesis of neurodevelopmental disorders." Schizophr Res 99(1-3): 56-70.

Fukada, M., A. Hanai, A. Nakayama, T. Suzuki, N. Miyata, R. M. Rodriguiz, W. C. Wetsel, T. P. Yao and Y. Kawaguchi (2012). "Loss of deacetylation activity of Hdac6 affects emotional behavior in mice." PLoS One 7(2): e30924.

Guillemin, G. J., B. J. Brew, C. E. Noonan, O. Takikawa and K. M. Cullen (2005). "Indoleamine 2,3 dioxygenase and quinolinic acid immunoreactivity in Alzheimer's disease hippocampus." Neuropathol Appl Neurobiol 31(4): 395-404.

Guillemin, G. J., G. Smythe, O. Takikawa and B. J. Brew (2005). "Expression of indoleamine 2,3-dioxygenase and production of quinolinic acid by human microglia, astrocytes, and neurons." Glia 49(1): 15-23.

Gulaj, E., K. Pawlak, B. Bien and D. Pawlak (2010). "Kynurenine and its metabolites in Alzheimer's disease patients." Adv Med Sci 55(2): 204-211. 
Halaris, A., A. M. Myint, V. Savant, E. Meresh, E. Lim, G. Guillemin, D. Hoppensteadt, J. Fareed and J. Sinacore (2015). "Does escitalopram reduce neurotoxicity in major depression?" J Psychiatr Res 66-67: 118-126.

Hass, U., O. Ladefoged, H. R. Lam, G. Ostergaard, S. P. Lund and L. Sinonsen (2001). "Behavioural effects in rats after prenatal exposure to dearomatized white spirit." Pharmacol Toxicol 89(4): 201-207.

Howland, J. G., B. N. Cazakoff and Y. Zhang (2012). "Altered object-in-place recognition memory, prepulse inhibition, and locomotor activity in the offspring of rats exposed to a viral mimetic during pregnancy." Neuroscience 201: 184-198.

Hughes, M. M., A. Carballedo, D. M. McLoughlin, F. Amico, A. Harkin, T. Frodl and T. J. Connor (2012). "Tryptophan depletion in depressed patients occurs independent of kynurenine pathway activation." Brain Behav Immun 26(6): 979-987.

Khan, D., P. Fernando, A. Cicvaric, A. Berger, A. Pollak, F. J. Monje and D. D. Pollak (2014). "Long-term effects of maternal immune activation on depressionlike behavior in the mouse." Transl Psychiatry 4: e363.

Lazic, S. E. and L. Essioux (2013). "Improving basic and translational science by accounting for litter-to-litter variation in animal models." BMC Neurosci 14: 37.

Maes, M., R. Yirmyia, J. Noraberg, S. Brene, J. Hibbeln, G. Perini, M. Kubera, P. Bob, B. Lerer and M. Maj (2009). "The inflammatory \& neurodegenerative (I\&ND) hypothesis of depression: leads for future research and new drug developments in depression." Metab Brain Dis 24(1): 27-53.

McQuown, S. C., R. M. Barrett, D. P. Matheos, R. J. Post, G. A. Rogge, T. Alenghat, S. E. Mullican, S. Jones, J. R. Rusche, M. A. Lazar and M. A. Wood (2011). "HDAC3 Is a Critical Negative Regulator of Long-Term Memory Formation." The Journal of neuroscience : the official journal of the Society for Neuroscience 31(2): 764-774.

Meyer, U., J. Feldon and S. H. Fatemi (2009). "In-vivo rodent models for the experimental investigation of prenatal immune activation effects in neurodevelopmental brain disorders." Neuroscience \& Biobehavioral Reviews 33(7): 1061-1079.

Meyer, U., J. Feldon, M. Schedlowski and B. K. Yee (2005). "Towards an immunoprecipitated neurodevelopmental animal model of schizophrenia." Neurosci Biobehav Rev 29(6): 913-947.

Mijovic, J. E., T. Zakar, D. B. Zaragoza and D. M. Olson (2002). "Tyrphostins inhibit lipopolysaccharide induced preterm labor in mice." J Perinat Med 30(4): 297-300. 
Morris, M. J., M. Mahgoub, E. S. Na, H. Pranav and L. M. Monteggia (2013). "Loss of histone deacetylase 2 improves working memory and accelerates extinction learning." J Neurosci 33(15): 6401-6411.

Mortensen, P. B., B. Nørgaard-Pedersen, B. L. Waltoft, T. L. Sørensen, D. Hougaard, E. F. Torrey and R. H. Yolken (2007). "Toxoplasma gondii as a Risk Factor for Early-Onset Schizophrenia: Analysis of Filter Paper Blood Samples Obtained at Birth." Biological Psychiatry 61(5): 688-693.

Myint, A. M. (2012). "Kynurenines: from the perspective of major psychiatric disorders." FEBS J 279(8): 1375-1385.

Myint, A. M., B. Bondy, T. C. Baghai, D. Eser, C. Nothdurfter, C. Schule, P. Zill, N. Muller, R. Rupprecht and M. J. Schwarz (2013). "Tryptophan metabolism and immunogenetics in major depression: a role for interferon-gamma gene." Brain Behav Immun 31: 128-133.

Nakamura, A., Y. Mori, K. Hagiwara, T. Suzuki, T. Sakakibara, T. Kikuchi, T. Igarashi, M. Ebina, T. Abe, J. Miyazaki, T. Takai and T. Nukiwa (2003). "Increased Susceptibility to LPS-induced Endotoxin Shock in Secretory Leukoprotease Inhibitor (SLPI)-deficient Mice." The Journal of Experimental Medicine 197(5): 669-674.

Nemeth, H., J. Toldi and L. Vecsei (2005). "Role of kynurenines in the central and peripheral nervous systems." Curr Neurovasc Res 2(3): 249-260.

Németh, H., J. Toldi and L. Vécsei (2006). Kynurenines, Parkinson's disease and other neurodegenerative disorders: preclinical and clinical studies. Parkinson's Disease and Related Disorders, Springer: 285-304.

Oxenkrug, G. F., W. Turski, W. Zgrajka, J. Weinstock, R. Ruthazer and P. Summergrad (2014). "Disturbances of Tryptophan Metabolism and Risk of Depression in HCV Patients Treated with IFN-Alpha." Journal of infectious disease and therapy 2(2).

Papafragkakis, H., M. Rao, M. Moehlen, S. Dhillon and P. Martin (2012). "Depression and pegylated interferon-based hepatitis C treatment." International Journal of Interferon, Cytokine and Mediator Research 4(1): 25-35.

Pinault, D. (2004). "The thalamic reticular nucleus: structure, function and concept." Brain Res Brain Res Rev 46(1): 1-31.

Piontkewitz, Y., Y. Assaf and I. Weiner (2009). "Clozapine administration in adolescence prevents postpubertal emergence of brain structural pathology in an animal model of schizophrenia." Biol Psychiatry 66(11): 1038-1046.

Raison, C. L., R. Dantzer, K. W. Kelley, M. A. Lawson, B. J. Woolwine, G. Vogt, J. R. Spivey, K. Saito and A. H. Miller (2010). "CSF concentrations of brain tryptophan and kynurenines during immune stimulation with IFN-alpha: 
relationship to CNS immune responses and depression." Mol Psychiatry 15(4): 393-403.

Renaud, S. J., T. Cotechini, J. S. Quirt, S. K. Macdonald-Goodfellow, M. Othman and C. H. Graham (2011). "Spontaneous Pregnancy Loss Mediated by Abnormal Maternal Inflammation in Rats Is Linked to Deficient Uteroplacental Perfusion." The Journal of Immunology 186(3): 1799-1808.

Rowe, D. L., P. A. Robinson, I. L. Lazzaro, R. C. Powles, E. Gordon and L. M. Williams (2005). "Biophysical modeling of tonic cortical electrical activity in attention deficit hyperactivity disorder." Int J Neurosci 115(9): 1273-1305.

Salminen, A., R. Paananen, R. Vuolteenaho, J. Metsola, M. Ojaniemi, H. AutioHarmainen and M. Hallman (2008). "Maternal endotoxin-induced preterm birth in mice: fetal responses in toll-like receptors, collectins, and cytokines." Pediatr Res 63(3): 280-286.

Sarkar, A., P. Chachra, P. Kennedy, C. J. Pena, L. A. Desouza, E. J. Nestler and V. A. Vaidya (2014). "Hippocampal HDAC4 contributes to postnatal fluoxetineevoked depression-like behavior." Neuropsychopharmacology 39(9): 22212232.

Snell, J. C., O. Chernyshev, D. L. Gilbert and C. A. Colton (1997). "Polyribonucleotides induce nitric oxide production by human monocyte-derived macrophages." J Leukoc Biol 62(3): 369-373.

Sørensen, H. J., E. L. Mortensen, J. M. Reinisch and S. A. Mednick (2009). "Association Between Prenatal Exposure to Bacterial Infection and Risk of Schizophrenia." Schizophrenia Bulletin 35(3): 631-637.

Tsankova, N. M., O. Berton, W. Renthal, A. Kumar, R. L. Neve and E. J. Nestler (2006). "Sustained hippocampal chromatin regulation in a mouse model of depression and antidepressant action." Nat Neurosci 9(4): 519-525.

Upton, K. J. and R. M. Sullivan (2010). "Defining Age Limits of the Sensitive Period for Attachment Learning in Rat Pups." Developmental psychobiology 52(5): 453-464.

Wichers, M. C., G. H. Koek, G. Robaeys, R. Verkerk, S. Scharpe and M. Maes (2005). "IDO and interferon-alpha-induced depressive symptoms: a shift in hypothesis from tryptophan depletion to neurotoxicity." Mol Psychiatry 10(6): 538-544.

Wischhof, L., E. Irrsack, C. Osorio and M. Koch (2015). "Prenatal LPS-exposure--a neurodevelopmental rat model of schizophrenia--differentially affects cognitive functions, myelination and parvalbumin expression in male and female offspring." Prog Neuropsychopharmacol Biol Psychiatry 57: 17-30.

Xu, D.-X., Y.-H. Chen, H. Wang, L. Zhao, J.-P. Wang and W. Wei (2006). "Tumor necrosis factor alpha partially contributes to lipopolysaccharide-induced 
intra-uterine fetal growth restriction and skeletal development retardation in mice." Toxicology Letters 163(1): 20-29.

Zhou, H. (2012). "Maternal Infection and Neurodevelopmental Disorders in the Offspring." American Journal of Immunology 8(1).

Zuckerman, L., M. Rehavi, R. Nachman and I. Weiner (2003). "Immune activation during pregnancy in rats leads to a postpubertal emergence of disrupted latent inhibition, dopaminergic hyperfunction, and altered limbic morphology in the offspring: a novel neurodevelopmental model of schizophrenia." Neuropsychopharmacology 28(10): 1778-1789.

Zuckerman, L. and I. Weiner (2005). "Maternal immune activation leads to behavioral and pharmacological changes in the adult offspring." $\underline{\mathrm{J} \text { Psychiatr }}$ Res 39(3): 311-323. 

Summary 
In this dissertation, we studied the role of inflammation in depression. Concretely, epigenetic changes after prenatal inflammation and the role of the kynurenine pathway in depression were investigated.

First, Chapter 1 provides an overview of the background of the disease, the current tools used for diagnosis and treatment, and the different pathophysiological theories related to depression. Moreover, the role of epigenetic mechanisms in the link between prenatal infection and depression in the offspring are addressed.

Chapter 2 represents a review about the current literature on how exposure to prenatal maternal infection may interfere with offspring brain development. More specifically, the immune system and the possible involvement of several mediators such as cytokines, neurotransmitters, neurotrophins, hormones and epigenetic processes are reviewed. Moreover, the effects of prenatal maternal stress and infection in the offspring, in view of inflammation, are discussed. Finally, the chapter ends with an overview of sex-differences in response to prenatal maternal infection as well as treatment options for major depression.

Chapter 3 describes the effects of lipopolysaccharide (LPS), an endotoxin contained in bacterial cell walls, on gestational length and intra-uterine and neonatal mortality in mice. The induction of prenatal inflammation using a high dose of LPS significantly decreased gestational length and increased neonatal mortality. When using a low dose of LPS, we observed a tendency 
towards reduced litter size. In addition, we discussed the advantages of using subcutaneous injections instead of intraperitoneal injections, when dealing with pregnant animals.

Chapter 4 analyses quinolinic acid (QUIN) in the naïve mouse brain in relation to affect-related behavior. We focused on the localization of QUIN in the naïve mouse because of the lack of knowledge regarding QUIN in healthy, control conditions. We showed QUIN-immunoreactivity particularly in the cingulate cortex (CC) delineating individual cells and in the thalamic reticular nucleus (TRN) resembling a fibrillary staining pattern. Moreover, we observed a strong correlation between QUIN-immunoreactivity in the TRN and motor activity in the open field test, suggesting a role of QUIN in the regulation of motor activity in normal conditions.

In Chapter 5 we assessed alterations in epigenetic mechanisms in the mouse brain after prenatal exposure to polyinosinic:polycytidylic acid (Poly I:C). More specifically, we examined global histone deacetylase (HDAC) and DNA methyltransferase (DNMT) activity within the brain. We found a significant sex $x$ treatment interaction effect after prenatal exposure to maternal immune challenge by Poly I:C, indicative of increased global HDAC activity particularly in female offspring from mothers injected with Poly I:C when compared to controls. In addition, the levels of the isoforms $\operatorname{HDAC} 1,2,3,4$ and 6 were explored as a possible explanation for the increase observed in global HDAC activity. However, we could not detect significant higher levels of the HDAC isoforms examined in Poly $\mathrm{I}: \mathrm{C}$ versus control female offspring. 
Although we observed a treatment effect with increased HDAC6 in offspring of mice treated with Poly I:C compared to controls, this effect seemed to be more pronounced in male - and not female - offspring, which does not match the global HDAC activity data. A feasible explanation for the discrepancy observed between the global HDAC activity and the protein levels of several HDAC isoforms in the present study is that one or more other HDAC isoform(s) than those assessed using western blotting is/are involved in mediating the observed increased in global HDAC activity. Taken together, this study indicates that prenatal maternal Poly I:C exposure seems to increase global HDAC, but not DNMT activity, particularly in female offspring. Altogether, though purely speculative, these data suggests a role for HDAC6 in mediating the association between prenatal maternal infection and the development of depression in the offspring.

Chapter 6 investigates alterations in the depression scores and in diverse enzymes and metabolites of the kynurenine pathway in Interferon-alpha (IFN- $\alpha$ ) treated patients. We report a significant change over time in both the Montgomery-Asberg Depression Rating Scale (MADRS) and the Beck Depression Inventory (BDI) scores during IFN- $\alpha$ therapy. In addition, levels of the neurotoxic metabolite kynurenine (KYN), 3-hydroxykynurenine (3HK), quinolinic acid (QUIN) and the kynurenine/tryptophan (KYN/TRP) ratio were increased over time, while the kynurenic acid/kynurenine (KYNA/KYN) ratio, reflecting neuroprotective properties, was reduced. Regarding the enzymes of the kynurenine pathway, the activity of kynureninase (KYNase), kynurenine-3-monooxygenase (KMO) and kynurenine aminotransferase 
(KAT-3) were also increased. When examining the relationship between MADRS scores and metabolites, KYNA and the KYNA/KYN ratio were negatively associated with MADRS scores. On the other hand, no association was observed between enzymes and the MADRS score. Finally, relative expression of KYNase significantly predicted levels of 3HK. Altogether, our results confirm, that the kynurenine pathway plays and important role in the development of depressive symptoms during IFN- $\alpha$ treatment, with a shift in the balance between neurotoxic and neuroprotective metabolites, as well as increased expression of enzymes regulating the neurotoxic arm of the pathway.

Finally, in Chapter 7, a summary and discussion of the main findings of this thesis are provided. In addition, some methodological considerations are addressed. Finally, concluding remarks are disclosed.

To sum up, in this thesis, we investigated possible mediators of the effects produced during brain development in offspring after prenatal inflammation, such as epigenetic mechanisms. In addition, we explored the role of QUIN in healthy conditions. Finally, we examined the role of several enzymes and metabolites of the kynurenine pathway in relation to depression in IFN- $\alpha$ treated patients. To conclude, we showed that prenatal maternal inflammation mediated changes in epigenetic marks, while exposure to cytokines in adulthood affected the kynurenine metabolism in relation to depression. 

Valorization 
Psychiatric disorders have a large impact on our society. More than 350 million people are estimated to suffer from depression including an increasing number of young persons. Concretely, major depression is the leading cause of disability with a high social, economic and societal burden (WHO 2012). Patients suffering from depression show a reduced capacity to study or work, which considerably affects the economy (Gilbody, Bower et al. 2012, McTernan, Dollard et al. 2013). Moreover, suicide and divorce rates are increased in depressed patients (Wolfersdorf 2008).

Major depression is a heterogeneous and complex disorder compromising a wide spectrum of symptoms, such as low mood, insomnia, fatigue and anhedonia among others (Kennedy 2008). There still is a lack of knowledge of the underlying cause of depression, which dramatically complicates its diagnosis and treatment. Diagnosis is still based on a clinical psychiatric interview, due to the lack of suitable biomarkers. Treatment consists mainly of antidepressants, which increase the concentration of monoamine neurotransmitters in the synaptic cleft. However, it is still unclear whether the therapeutic effect of antidepressants is related to their monoaminergic actions. For instance, while antidepressants rapidly increase monoamine levels, weeks or months of treatment are required before clinical improvement is observed. In addition, treatment efficiency is only 60-65\%, with a remission rate of around 30\% (Block and Nemeroff 2014), and at least $20 \%$ of depressed patients are treatment-resistant (Berlim and Turecki 2007). In view of the high societal burden, it is vital to deepen our understanding of the biological mechanisms of depression in order to unveil new potential therapeutic targets, such as the kynurenine pathway. 
Depression has been linked to inflammation, since a subgroup of depressed patients show higher circulating levels of pro-inflammatory cytokines (amongst other increased inflammatory markers), and major depression is frequently comorbid with many inflammatory diseases (Anisman and Hayley 2012, see review by Gray and Bloch 2012). Interestingly, cytokines regulate several enzymes of the kynurenine pathway (Myint 2012), leading to the production of several neurotoxic, e.g. quinolinic acid (QUIN), metabolites. Thus, the kynurenine pathway has been considered as a possible mechanism involved in the pathophysiology of depression (Maes, Mihaylova et al. 2007, Myint, Kim et al. 2007, Miura, Ozaki et al. 2008) and its metabolites and enzymes may serve as potential biomarkers (Fukuda 2014).

Many studies have examined the levels of different metabolites of the kynurenine pathway in depressed patients (Sublette, Galfalvy et al. 2011, Ogawa, Fujii et al. 2014, Savitz, Drevets et al. 2015). However, the full range of metabolites in conjunction with the expression of genes involved in the kynurenine pathway has not been examined yet. In this thesis, we show increased levels of some considered neurotoxic metabolites of the pathway e.g kynurenine (KYN), 3-hydroxykynurenine (3HK) and QUIN, as well as increased expression of the enzymes regulating this arm of the pathway. In addition, the negative association observed between the kynurenic acid/kynurenine (KYNA/KYN ratio and scores in the Montgomery-Asberg Depression Rating Scale (MADRS), underscores that development of depressive symptoms is mediated by a disturbance in neuroprotective support. Altogether, we found altered levels of neurotoxic and 
neuroprotective metabolites in IFN- $\alpha$ treated patients, consolidating the possible use of these metabolites as biomarkers for depression.

In particular, QUIN has been studied extensively, because of its known neurotoxic effects (Nemeth, Toldi et al. 2005). Although physiological levels of QUIN are necessary in healthy conditions to synthetize nicotinamide adenine dinucleotide (NAD), which is needed for any cell to function properly (Myint 2012), evidence is lacking regarding QUIN in normal physiological conditions. Therefore, in Chapter 4 we investigated in the brain of naïve mice the localization of QUIN, the pattern of its staining, and its possible association with a subset of affect-related behavioural phenotypes. We detected QUIN-immunoreactivity, particularly in the cingulate cortex and the thalamic reticular nucleus (TRN), and it was strongly correlated with locomotor activity in males. This suggests an important role for QUIN at the level of the TRN in regulating motor behaviour. Interestingly, the TRN has been involved in attention-deficit hyperactivity disorder (ADHD), specifically in the modulation of arousal (di Michele, Prichep et al. 2005, Rowe, Robinson et al. 2005). In addition, recently a study reported than the QUIN/3HK ratio was positively correlated with psychomotor agitation in depressed subjects (Halaris, Myint et al. 2015). To sum up, we show that QUIN is observed in normal conditions in the brain and that it may play a role in the regulation of motor behavior. Thus, the conception of QUIN just as a neurotoxic factor should be further clarified.

Another mechanism that has been associated with depression is epigenetic programming. Specifically, changes in histone acetylation have been involved in the pathophysiology of depression (Tsankova, Berton et al. 
2006). In addition, histone deacetylase (HDAC) inhibitors have been reported to exert antidepressant effects (Yamawaki, Fuchikami et al. 2012, Hsing, Hung et al. 2015) and have been suggested as possible targets for the future development of novel antidepressant treatments (Covington, Maze et al. 2009). Therefore, we studied changes in epigenetic processes in a maternal immune activation mouse model for depression using a viral mimetic, e.g polyinosinic:polycytidylic acid (Poly I:C). We observed an increase in global HDAC enzymatic activity, particularly in females' offspring exposed prenatally to Poly I:C. Further investigations on the specific HDAC isoforms involved and the effects produced by HDAC inhibitors, would be of great value.

To conclude, this thesis aimed to provide more evidence on the current approaches to diagnose and treat depression. Our results are certainly of interest to subjects undergoing IFN- $\alpha$ or pro-inflammatory cytokine therapy and to depressed patients suffering from an inflammatory condition. To our knowledge, we are the first to analyse the complete KYN pathway in conjunction with the gene expression levels of the enzymes involved, in IFN$\alpha$ induced depression. Besides, we combined the measurements of global DNA methyltransferase (DNMT) and HDAC activity with specific HDAC isoform measures. Our studies support further investigation in the use of HDAC inhibitors as new targets for the treatment of depression. In addition, we reaffirmed the use of metabolites of the kynurenine pathway, e.g. KYN, 3 HK and QUIN and the enzymes regulating this neurotoxic arm, kynureninase (KYNase) and kynurenine-3-monooxygenase (KMO), as 
potential biomarkers for the diagnosis of depression. These metabolites and enzymes mentioned above could also be used to differentiate depressed patients with or without underlying inflammatory conditions. In addition, their measurement before and after treatment, might help in the prediction of the therapeutic response. 


\section{References}

Anisman, H. and S. Hayley (2012). Inflammatory Factors Contribute to Depression and Its Comorbid Conditions.

Berlim, M. T. and G. Turecki (2007). "Definition, assessment, and staging of treatment-resistant refractory major depression: a review of current concepts and methods." Canadian Journal of Psychiatry 52(1): 46.

Block, S. G. and C. B. Nemeroff (2014). "Emerging antidepressants to treat major depressive disorder." Asian Journal of Psychiatry 12(0): 7-16.

Covington, H. E., 3rd, I. Maze, Q. C. LaPlant, V. F. Vialou, Y. N. Ohnishi, O. Berton, D. M. Fass, W. Renthal, A. J. Rush, 3rd, E. Y. Wu, S. Ghose, V. Krishnan, S. J. Russo, C. Tamminga, S. J. Haggarty and E. J. Nestler (2009). "Antidepressant actions of histone deacetylase inhibitors." J Neurosci 29(37): 11451-11460.

di Michele, F., L. Prichep, E. R. John and R. J. Chabot (2005). "The neurophysiology of attention-deficit/hyperactivity disorder." Int J Psychophysiol 58(1): 8193.

Fukuda, K. (2014). "Etiological classification of depression based on the enzymes of tryptophan metabolism." BMC Psychiatry 14: 372.

Gilbody, S., P. Bower and J. Rick (2012). "Better care for depression in the workplace: integrating occupational and mental health services." The British Journal of Psychiatry 200(6): 442-443.

Gray, S. M. and M. H. Bloch (2012). "Systematic Review of Proinflammatory Cytokines in Obsessive-Compulsive Disorder." Current psychiatry reports 14(3): 220-228.

Halaris, A., A. M. Myint, V. Savant, E. Meresh, E. Lim, G. Guillemin, D. Hoppensteadt, J. Fareed and J. Sinacore (2015). "Does escitalopram reduce neurotoxicity in major depression?" J Psychiatr Res 66-67: 118-126.

Hsing, C.-H., S.-K. Hung, Y.-C. Chen, T.-S. Wei, D.-P. Sun, J.-J. Wang and C.-H. Yeh (2015). "Histone Deacetylase Inhibitor Trichostatin A Ameliorated Endotoxin-Induced Neuroinflammation and Cognitive Dysfunction." Mediators of Inflammation 501: 163140.

Kennedy, S. H. (2008). "Core symptoms of major depressive disorder: relevance to diagnosis and treatment." Dialogues in Clinical Neuroscience 10(3): 271277.

Maes, M., I. Mihaylova, M. D. Ruyter, M. Kubera and E. Bosmans (2007). "The immune effects of TRYCATs (tryptophan catabolites along the IDO pathway): relevance for depression - and other conditions characterized by tryptophan depletion induced by inflammation." Neuro Endocrinol Lett 28(6): 826-831. 
McTernan, W. P., M. F. Dollard and A. D. LaMontagne (2013). "Depression in the workplace: An economic cost analysis of depression-related productivity loss attributable to job strain and bullying." Work \& Stress 27(4): 321-338.

Miura, H., N. Ozaki, M. Sawada, K. Isobe, T. Ohta and T. Nagatsu (2008). "A link between stress and depression: shifts in the balance between the kynurenine and serotonin pathways of tryptophan metabolism and the etiology and pathophysiology of depression." Stress 11(3): 198-209.

Myint, A. M. (2012). "Kynurenines: from the perspective of major psychiatric disorders." FEBS Journal 279(8): 1375-1385.

Myint, A. M., Y. K. Kim, R. Verkerk, S. Scharpe, H. Steinbusch and B. Leonard (2007). "Kynurenine pathway in major depression: evidence of impaired neuroprotection." J Affect Disord 98(1-2): 143-151.

Nemeth, H., J. Toldi and L. Vecsei (2005). "Role of kynurenines in the central and peripherial nervous systems." Current neurovascular research 2(3): 249260.

Ogawa, S., T. Fujii, N. Koga, H. Hori, T. Teraishi, K. Hattori, T. Noda, T. Higuchi, N. Motohashi and H. Kunugi (2014). "Plasma L-tryptophan concentration in major depressive disorder: new data and meta-analysis." J Clin Psychiatry 75(9): e906-915.

Rowe, D. L., P. A. Robinson, I. L. Lazzaro, R. C. Powles, E. Gordon and L. M. Williams (2005). "Biophysical modeling of tonic cortical electrical activity in attention deficit hyperactivity disorder." Int J Neurosci 115(9): 1273-1305.

Savitz, J., W. C. Drevets, B. E. Wurfel, B. N. Ford, P. S. Bellgowan, T. A. Victor, J. Bodurka, T. K. Teague and R. Dantzer (2015). "Reduction of kynurenic acid to quinolinic acid ratio in both the depressed and remitted phases of major depressive disorder." Brain Behav Immun 46: 55-59.

Sublette, M. E., H. C. Galfalvy, D. Fuchs, M. Lapidus, M. F. Grunebaum, M. A. Oquendo, J. John Mann and T. T. Postolache (2011). "Plasma kynurenine levels are elevated in suicide attempters with major depressive disorder." Brain, Behavior, and Immunity 25(6): 1272-1278.

Tsankova, N. M., O. Berton, W. Renthal, A. Kumar, R. L. Neve and E. J. Nestler (2006). "Sustained hippocampal chromatin regulation in a mouse model of depression and antidepressant action." Nat Neurosci 9(4): 519-525.

WHO (2012). "Depression: A Global Crisis. World Mental Health Day, October 10 2012. World Federation for Mental Health. Available: http://www.who.int/mental health/management/depression/wfmh pap er depression wmhd 2012.pdf."

Wolfersdorf, M. (2008). "[Depression and suicide]." Bundesgesundheitsblatt Gesundheitsforschung Gesundheitsschutz 51(4): 443-450. 
Yamawaki, Y., M. Fuchikami, S. Morinobu, M. Segawa, T. Matsumoto and S. Yamawaki (2012). "Antidepressant-like effect of sodium butyrate (HDAC inhibitor) and its molecular mechanism of action in the rat hippocampus." World J Biol Psychiatry 13(6): 458-467. 

Acknowledgements 
I would like to thank all the people that in one way or another contributed to make this thesis possible.

First of all, thank you to Aye-Mu Myint. I still remember the day when you informed me that I was accepted to this project. Although I came from a different background, you believed in me and gave me the opportunity to work in this field. You taught me not only about science, but also about life. We had great moments living together, at the office, in conferences, at dinners, travelling or even just discussing! I hope you are enjoying the peace that you were always looking for. Thanks, again, for sharing your knowledge with me.

Next, I would like to thank all the other seniors and professors that accompanied me during my stay in Munich: Norbert Muller, Markus Schwarz, Brigitta Bondy, Peter Zill, Sylvia de Jonge, Karin Neumeier and Brian Leonard. I appreciate all your guidance, suggestions, support, input and collaboration.

I give my heartfelt gratitude to Ines Bolle teaching us how to handle mice, helping us with the animals and sharing your knowledge with us. To the technicians: Ursula and Heidi. Thanks for teaching me superior lab skills and for all your help and assistance. To all my officemates of the Nußbaumstrasse: Paolo, Ozden, Mamun and Marta. Paolo, we shared a lot of moments, beginning in the office, having nice dinners and parties in Munich, in conferences and, of course, Oktoberfest. I still remember that we could communicate speaking catalan and calabrese. Grazie di tutto. Ozden thanks also for the great moments and, specifically, guiding me around of 
Istanbul last year! Also to Mamun and Marta, por esos buenos momentos que pasamos en el laboratorio empapadas y por esa gran fiesta de carnaval!!

Andrea und Waldtraud: Ich habe so viel von euch gelernt, vielen Dank für alles! Wir haben sehr schöne gemeinsame Momente erlebt, unter anderem gemeinsam zu Abend gegessen und die Wiesn besucht. Ihr habt viel zu diesem Projekt beigetragen. Ich schätze euer großes Interesse, das ihr gezeigt habt, obwohl ich schon in Maastricht war. Wir treffen uns sicherlich in München, wenn ich zu Besuch sein werde. Herzlichen Dank!

Zuletzt mein PhD-Kollege Andy: Es ist nicht einfach zu beschreiben, wie dankbar ich dir bin. Wir haben dieses Projekt zusammen begonnen. Du hast mir auch viel beigebracht. Ich glaube, dass wir immer ein gutes Team waren. Wir haben viele tolle Momente erlebt (im Laboratorium, auf Geburtstagsfeiern, Konferenzen, Reisen, beim Abendessen...) und auch einige schwierige. Aber für mich war der schwerste Moment, als du mir gesagt hast, dass du nicht nach Maastricht fahren würdest. Es war nicht einfach für mich, deine tägliche Unterstützung zu verlieren. Aber ich wusste, dass wir auf jeden Fall in Kontakt bleiben würden. Und so ist es auch. Ich wünsche dir das Beste, du wirst ein wunderbarer Lehrer sein! Wir sehen uns bald. Vielen, vielen Dank für alles!

This thesis would have been not possible without the support of Professor Harry Steinbusch. You gave me the opportunity to come to Maastricht, to continue and finish my PhD here. Your advice and ideas were always of great value for the project. You showed me to be critical about my research and to think in a global manner. Thanks for your interest in this project. 
Thank you to my daily supervisors: Bart, Gunter and Daniel. Bart, thanks for your feedback and your support, especially in the beginning of my project. Not only did you guide me with my work, you also inquired about how things were going with my new life in Maastricht. Gunter, you incorporated to the project later on, but provided advice and guidance. I really enjoyed our weekly meetings and discussions with Daniel. I also appreciate a lot your support, especially during this last period of the PhD. Last, but not least, many thanks to Daniel. You supported me from the fruition of the project until the very end. I imagine that it was not easy for you to face all the new challenges of this project, in which you were unexpectedly involved. You showed me that there is always a solution for all the problems. It was a pleasure for me to have you as a supervisor. It is very easy to work with you, always with a smile in your face and your good mood. You always gave me energy and really valuable suggestions and feedback. I grew as a scientist next to you and I learned a lot from you. Thanks for your guidance. This thesis would have not been possible without you.

I would like to thank also Marc, as a chairman of my reading committee, for your efficiency and feedback about this thesis.

Many thanks to the seniors of the department: Jos, Yasin, Pilar, Mario, Fred and Govert, and Jochen and Ali for your valuable feedback in any occasion.

I would also like to thank to Ankie Hochstenbach, Marjo Zwanenburg, Martie Wanders, Marie Therese, Lisa and Rachelle. You were always ready to help me with all my questions about forms and the many issues us foreigners have to deal with. 
To the team of wonderful technicians (Hellen, Marjan, Denise and Barbie): many thanks for all your help and all your wealth of information. You were always there to answer all my "short" questions and to share with me your knowledge about western blots, qPCR and stainings. It was very nice to work with you. And also to Wiel, many thanks to deal with all my computer's problems!!

To my officemates: Maarten, Laurence, Gisela, Nynke and Koen. I really enjoyed your company as my officemates. Laurence, thanks for all your help, you were always available to answer to all my differing questions, it was a pity when you had to move from our room $\otimes$. Nynke, thanks for your time, and for your interest in how I was doing, especially during the last period. Koen! I laughed a lot with you during these months, it was nice to share with you time in the office but also outside in the many picnics and bbqs. As a reward, I gave you as an inheritance my fantastic office chair!!! Take care of it jajajaja!

And my paranymphs: Maarten, you were my first officemate during this PhD. We shared many moments in the office, but also picnics, dinners, parties and carnival!! Since I never saw you closing the Alla, this will be your main duty as my paranymph jajajaja!! Good luck with the rest of your PhD, although you are already recognized as a Doctor in some interviews;-)

Gisela: que bien tener a una compañera de oficina de casa!!!! Me encanto compartir la oficina contigo y aprendí mucho de ti!!! Gracias por todos esos consejos sabios que me has dado y por todo el ánimo y apoyo estando en Maastricht y ya de vuelta en casa. Por todos esos momentos vividos y los que vendrán!! Te echamos de menos!!!!!!!!!!! 
To the TNP group- Nicole, Mark, Roy, Ehsan, Artemis, Marion! And also to Yuan! Here's to the funny meetings and going out activities!!!

Roy: many thanks for your help, to my many questions about PCR and stainings, even when you were in Boston!!!! A gold strike is waiting for you in my defense party!!

Ehsan: thanks for all your statistics input and your predisposition to help. It was a pleasure to work with you and good luck with your defense and the post-doc life!!

Artemis: we needed someone like you!!! With all this energy to organize picnics and dinners, bbqs and the birthday party!! It was nice to have you in the group, a pity that was in my last period. Good luck with your project!!

Caroline: also a pleasure to share with you many lunches, picnics and dinners, as well as our birthday party!! Also good luck with your project!

Shengua: you arrived only recently to the department, but we could already enjoy some funny moments. Also good luck with your project!

Gerard: you were leaving not long after I arrived, but still we could share some beers!! You will be also a Doctor soon!! Though it took a little bit more than expected!!

Joao: the Portuguese one! We did not work together, but still we could share funny moments!! Good luck with your PhD and now that you have whats app it will be easier to keep the contact jajajaja!

Marina: la última incorporación!!!! Nunca me pensé que tendría a otra tarragonina en el departamento jajaja! Un placer tenerte como amiga, espero que los 3 años que te quedan en Maastricht sean llevaderos jajaja! A 202 
organizar muchas más calçotadas!! Y cuidado con los pimientos!!! A ti también te quiero ver como a Maarten hasta el cierre en el Alla :)

Many thanks to my students Ceciel and Max: part of this project is also yours. It was a pleasure to work with you, thanks for your motivation, dedication and hard work. I am sure that you will become two amazing scientists!!!

To the international dinner group: Joost, Sanne, Mehrdad, Paula, Giacomo, Nevena, Gabri, Shuan, Martin, Thessely, Artemis, Sean and Elisa! For all the amazing dinners that we had as well as the new constitution!!!!

Now to my missed ones:

Julie: you were one of my first friends in Maastricht, and the one who introduced me here to the social life. We had a lot of moments together that will be difficult to forget. It was an honor for me to be your paranymph! It was also very nice to see you in California and back here in Maastricht. Thanks for all and waiting for the big announcement to go back to the States ;)

Alejandro: vecinooooo! Por aquí te echamos de menos!!! Un placer haber compartido cenitas, fiestas, Oktoberfest, y como no carnavallll (sin ti no es lo mismo...). Buenísimos los días que pasamos en LA y mi querido San Francisco, ahora es tu turno de visitarnos, que ya estas tardando mucho! Aquí te esperamos con los brazos abiertossss!!!!

Y como no a mi otra vecina Paula! Por esas cenitas, la lavadora prestada, carnavales, Oktoberfests... fue raro ya no tenerte de vecina! 
Mucha suerte en esta nueva etapa que has empezado con tantos cambios y habrá que mudarse de Maastricht no???

Y ahora a los españolitos del pueblo:

Elena: a ti fui la primera a la que conocí. Muchas gracias por todos tus consejos de integración en este país jajaja y por tu interés en mi tesis. Se me hará raro el no tener esas tardes de café y charlas, pero las seguiremos teniendo donde sea que este. Mucha suerte con este cambio, seguro va a ser para mejor! Te lo merecías!!! Besosssss!

Cris: mi granainaaaaa!! También un placer el haber tenido tu compañía durante este tiempo en Maastricht y el compartir nuestras alegrías y penas, sobre todo relacionadas con el tiempo y encontrar un sitio donde poder picar algo jajaja! Curioso ver cómo han cambiado las cosas desde que nos conocimos, pero bueno ha sido para bien así que sin quejarse jaja! Te echare de menos! Muaaaaaaaaa

Fran, Patri y Juan Gonzalo: a vosotros os escribo a los 3 juntos jajaja. Fue un placer teneros por aquí durante un tiempo y una lástima que os marcharais, pero bueno sé que no estáis sufriendo jajaja! Os deseo toda la suerte del mundo y a ver si nos vemos prontoooooo!!!

Victor: a ti también te conocí al principio y me ayudaste a conocer más gente. Muy buenos momentos compartidos y partidos de futbol!!! Habrá que verse más a menudo!!! También te deseo lo mejor y seguro te irá bien!!

Victoria: mi malagueñaaaaa! Aun me acuerdo cuando nos dijiste que te ibas de Maastricht.... Aun no me lo creo! Pero bueno sé que estas genial 204 
y me alegro de que todo te vaya bien! Fue un placer verte en Málaga y a ver si esperamos tanto para la próxima!! Besossssss! El helado buenísimo todavía me acuerdo jajaja!

Noelia: también un placer haber compartido mi vida en Maastricht contigo y muy feliz de haberte visto, sabiendo que todo te va genial. Y así seguirá, seguro porque te lo mereces! Besos guapa!

Jaimeeeeeeeee: mi loco Jaime! Quien me iba a decir a mi cuando te conocí vestido de cordobés jajajaja! Contigo es imposible no reírse, gracias por todos los momentos vividos, carnavales, en colonia, en Maastricht y como no esas Fallas en Valencia, vamos que repetimos el próximo año!!! Al caloret!!! Besazoooooooo guapo!!!

Miguel Angel: el meu català! Encara al-lucino amb el teu nivell!! També un plaer haver-te conegut i disfrutat molts moments i viatges amb tu! Seria molt bo si ens trobéssim a Munic jajaja, pero sino serà allà on estiguem! Petons macoooooo!

Sofia: la meva altra catalana! També amb tu es impossible parar de riure! Gràcies també pel teu interès i per tots els moments viscuts i els que ens queden!! Quan has dit que inaugurem la nova casa, jajajaja!!! Molts petons!! I tenim un viatge pendent a Geòrgia!!

Y ahora mis dos pilares, sin vosotros Maastricht habría sido muy muy diferente.....

Carlos: my PhD soulmate! Madre mía, quién nos mandó meternos en este follón! Tantas charlas sobre eso... pero al final se sale, y tú también lo harás pronto y te saldrá genial como crack que eres!! Muchas gracias por todo, buenos y malos momentos y por todas esas organizaciones de viajes y 
cenas y fiestas, increíble!!! Mucha suerte con la fase final y no te vayas muy lejos que las fiestas sin ti no son lo mismo jajajaja! Muchos besossssssss!

Nienke: muy difícil escribir aquí tantas cosas que hemos vivido. Tú sabes que yo no soy de palabras pero gracias por tu amistad. Me lo he pasado genial contigo!! Cuando te conocí pensé por finnnnn alguien con el mismo ritmo jajajaja! También por los buenos y malos momentos vividos, todos los viajes, nuestros días en Estambul, y los que nos quedan pendientes. Por todas esas risas y las que nos quedan. Espero que no nos vayamos muy lejos la una de la otra, que si no te echaré mucho de menos!!! Besazos pelirrojaaaaaaaa!

Muchas gracias también a Santi, Cris, Dani y Juanmi, por vuestro interés y por todas esas noches locas para desconectar. Cuándo es la próxima??

Now going back in time. I have still to thank a lot of people before this project began.

Marta, como siempre digo mi amiga de toda la vida (:). Quien me hubiera dicho que desde esos momentos cuando nos conocimos en el comedor del colegio ibas a ser mi gran apoyo. Creo que tú eres la persona que mejor me conoce después de todos los años que hemos pasado juntas. Muchas gracias por tu apoyo, siempre estuviste ahí cuando lo he necesitado. Y siempre me has sorprendido viniendo a verme a donde fuera! Tantos momentos compartidos, risas y llantos, y todos los que aún nos quedan por vivir, porque si algo tengo claro es que siempre te tendré ahí, y tu pronto me tendrás en Cambrils Resort con Lina jajajaja, estoy ya soñando con las 206 
barbacoas, los mojitos y las paellas en la playa, aixxxx que ganassssssss! Gracias por todo!! Y que conste que Teruel existe!

Mis amigas de la Autónoma. Mis biólogas: Alba, Cris y Vero, que deciros después de tantos años de amistad (12 ya madre mía!!!!). Nunca olvidare todas las aventuras que hemos vivido juntas, desde las excursiones de botánica, partidas de cartas en la cafetería, nuestras comidas en el mandri, las noches de fiesta en Barcelona, los viajes que hemos hecho juntas, y que no han sido pocos jajaja! Os estoy muy agradecida de vuestra amistad, nunca pensé que pudiera conseguir tan grandes amigas en la universidad y lo mejor es que sé que todavía nos quedan muchas cosas juntas por vivir.

Alba: bueno Dra. Martinez mejor, gracias por acogerme en tu estupendo pisito en BCN, desde que ya no vivo allí. Me alegro mucho de haberte conocido y de todos los buenos momentos que hemos vivido. Espero que pronto encuentres algo donde puedas demostrar tu valía que es mucha! Y no te nos vayas de BCN, que alguna tiene que quedarse!!!

Cris: tantas cosas hemos vivido y eso que en los últimos anos nunca hemos vivido en el mismo país jajajaja! Siempre ha dado igual donde haya estado la una y la otra, mejor así teníamos excusas para viajar y encontrarnos donde fuera. Desde la universidad, tu etapa en Cork, nuestra aventura en California, mi mudanza a Múnich, y luego con tu ayuda a Maastricht, numerosos festivales jajaja y la última escapada a UK, no sabría decir todo lo que hemos reído, comentado e imaginado. Nunca sabremos donde nos llevará el futuro (espero que no muy lejos), pero lo que si sé es que donde sea nos encontraremos!! 
Vero: la meva suposada germana jajaja! Vas ser més que una amiga durant la nostra etapa a la Autònoma i vaig gaudir molt dels mesos que vam viure juntes, de les escapades a Vilafortuny i de la teva visita a Munic i a Maastricht. Ara no et tinc més a prop, però estic contenta perquè sé que ets feliç i perquè sé que encara ens continuarem veient, encara que no sigui tan sovint. Torna a Europaaaaaaaaa!!!

Y aquí reservo un espacio para Verónica también. Te conocí un poco más tarde en San Francisco, pero eres como una compi más de la universidad. Muchas gracias por todos esos descuentos en super hoteles y por todos esos viajes geniales donde tanto hemos conocido y disfrutado y todos los que nos quedan por hacer! También por tus visitas a Múnich y Maastricht! Próximo destino?????

Y también Andrea! Tu eres la encargada de cerrar el círculo de PhD! Muchas gracias también por acogerme siempre y por todas esas fiestas y charlas! Nos vemos en tu defensa!!!!

També a tu Carol. Mai oblidaré la oportunitat que hem vau donar de fer les meves primeres pràctiques, de trepitjar per primera vegada un laboratori real, de tot el que em vas ensenyar. Sé que vas treballar molt dur per aconseguir el treball dels teus somnis, i admiro que encara ara després de tants anys no hagis perdut la il-lusió i sapiguis contagiar-la als teus estudiants. I lo millor es que encara poguem fer un Viena de tant en tant després de tants anys i posar-nos al dia! T'estaré sempre agraïda!!!

I com no a l'Anna. La meva profe d'alemany! Tu vas ser qui em va donar l'empenta per marxar de casa i anar-me'n a provar sort a Alemanya, $\mathrm{i}$ concretament a Munic. I això ha canviat la meva vida totalment. Moltes 208 
gràcies per compartir amb nosaltres el teu entusiasme per Alemanya, això si sempre sent realistes jajajaja! El tenir-te a tu com a professora feia molt més simple aprendre l'idioma! Et desitjo tot lo millor a Berlin o a on sigui!

També unes linies pel David. Qui ens ho hauria dit que després del curs d'alemany compartiriem tants cafès i orxates? Jajajaja! Moltes gràcies pels teus emails, sempre preocupant-te per com estava i per l'estatus de la meva tesi. Aviat ens veiem!!!

Y ahora llego el momento de Múnich. Gracias a toda esa familia enorme, como ya he dicho alguna vez, uno no sabe que hay dos tipos de amistad, hasta que se muda al extranjero, y gracias a vosotros, yo lo he podido saber. Primero a mis compis del curso de alemán: Marion y Salvatore.

Marion, muchas gracias por toda tu ayuda, fue para mí una gran suerte conocerte nada más llegar a Múnich. Lo pase genial todas esas noches en el patio, y por todos los demás momentos que hemos compartido, incluida tu boda. Ahora no nos vemos tan a menudo, pero sé que nos iremos viendo igualmente!!

Salvatore!!! Que decirte! Gracias por tu amistad, por ensenarme Múnich, por darme tantos consejos y por hacer de tus amigos mis amigos! También por todos esos viajes de fin de año y los que nos quedan aún! Te debo todavía una visita a Nápoles jajaja! Próximamente.... Grazie mille!!!

Edgar, uno de mis weyes! Pues nunca pensé que llegarías a ser tan gran apoyo para mí. Contigo siempre puedo hablar las cosas tal y como son, y valoro mucho la sinceridad entre nosotros. Muchas gracias por todos esos momentos en Múnich, que no han sido pocos, desde futbol, a fiestas, 
Oktoberfest, bueno todo lo que acabe en fest jajajaja, bodas, viajes, domingos santos de películas y charlas, sobretodo nuestras charlas. Sabes que siempre puedes visitarnos en Reus (in-de, independencia jajajaja) y a mi allá donde este. Queda pendiente nuestro viaje a México!!!

Berny, mi otro wey, además de vecino jajaja!! Que decirte, también fue un poco sorpresa para mí nuestra amistad. Fuiste uno de los últimos que llegaste al grupo, pero supiste como hacerte un espacio. Gracias por todo tu apoyo y todos esos momentos que hemos compartido, desde fiestas a desayunos, partidos, visitas a Saturn, viajes y terracita, esa terracita en Múnich jajaja! Fue difícil el asimilar que te ibas tan lejos, pero como ya imaginaba, eso tampoco ha cambiado nada. Me debes una visita al pueblo!!!! Ves pensando donde nos encontramos para fin de año! Que somos los únicos fijos!!! Gracias por todo!!!

Sarah y Stephan: gracias por acogerme siempre que lo he necesitado. Sarah, a ti especialmente por ser mi primer tándem, siempre dispuesta a corregir mi alemán y por tu generosa ayuda a mejorar mi currículum y aconsejarme en todo lo que yo no sabía. Probablemente, sin ti esta tesis tampoco hubiera sido posible. Herzlichen Dank! Stephan, a ti también! Por esos cumples, fiestas y todas esas cerves y tardes de futbol! Me encantaron!!!!

Livia y Bianca: gracias también a vosotras. Por esas vacaciones en Mallorca e Ibiza, siempre un placer organizar estos viajes con vosotras. Y por tantos momentos en cumpleaños y en el Wiesn. Os deseo toda la felicidad y espero veros pronto!!! 
Gisela y Frank: esas fiestas de Halloween, increíbles!! Gracias también por los momentos compartidos, días de esquí, cenitas, fiestas y viajes!! Os deseo todo lo mejor en Múnich.

Lidia y Jorge: mis madrileños jajaja! A vosotros también os conocí al principio y hemos pasado momentos geniales. Ese día de churros! Y paella! Y comidas olímpicas! Y wiesn y tantas cosas que seguro me olvido más de la mitad!!! Jorge muchas gracias por tus consejos informáticos y por esos preciosos calendarios, y Lidia que sería de mí y de spss sin ti jajajaja! Muchas gracias $\mathrm{x}$ siempre estar ahí para responder a mis preguntas estadísticas y por todos tus consejos! Me debéis una visita al pueblo!! Jajajaja!

David, Tomomi y Kokoro: familiaaaaaaaaaa!!! A vosaltres us vaig conéixer al final, però encara i així hem tingut temps per compartir molts moments!! Estic encantada de veure-us tant feliços!! Per cert la xocolata i el fuet bonissims jajaja!!

Luis: gracias a ti también por tantos momentos en Múnich y tantas tardes de futbol!!! Fue una pena que nos dejaras pero me alegro que todo te vaya bien en Suiza. Muchas gracias por tu visita a Maastricht y nos vemos pronto en Múnich!

Zuri: juntas empezamos nuestra aventura en Múnich y descubrimos esa ciudad. Estuviste conmigo desde el principio, todavía recuerdo aquella lista de qué hacer para encontrar mi trabajo que hicimos en un Biergarten jajaja! Gracias por tu apoyo siempre y por todos los momentos que hemos disfrutado juntas, desde vacaciones como olvidar Croacia, en Reus, y los días en Maastricht, cenas, wiesn, cerves, fiestas, mudanzas, cafés, charlas y todo lo que nos queda. Eskerrik asko!!! 
Ricci: Ich war so froh, als ich dich kennengelernt habe! Ich wusste schon, dass wir sehr gute Freunde sein würden, und so kam es auch! Wir haben viele gemeinsame Momente erlebt: Urlaub in Italien, Portugal, Kroatien, Sizilien, Reus und auch in Maastricht. Auch die Zeit in unserem Lieblingsort München haben wir sehr genossen, unter anderem die vielen Partys und natürlich die Wiesn! Und wie sehr ich dein Essen genossen habe! Ich hoffe, dass ich dich in Australien besuchen kann. Ich weiß, dass du immer da bist, wenn ich dich brauche. Grazie mille! Wir sehen uns in München!

Giselita: mi portuguesa colombiana. Muchas gracias también por todo! Desde que te conocí en la escuela de alemán, todo lo que hemos vivido, ese queso fundido en el patio, las noches en Latinos ai ai ai!! Y esos días en Portugal, esos fines de año fantásticos y tantas cosas más que necesitaría muchísimas páginas para escribir. Me debes todavía una visita a Reus!!! Muchos besossssssssssssss!

Mariela: ai Mariela quien nos iba a decir a nosotras en el curso de alemán todo lo que nos venía por delante jajajaj! Desde Reus a Múnich, viviendo en nuestros bunkers, disfrutando del Englischer Garten y del wiesn! También por tu visita a Maastricht! Gracias por acogernos en tu pisito en el centro y por tu amistad! Nos vemos en Reus, Múnich o donde sea!!! Muaaaaaaaaaaaaaaa.

Rocio y Sergio: Gracias a los dos por acogerme también en vuestra casa a mí y a Nienke y a Carlos y a quien hiciera falta jajaja, y por vuestras visitas a Maastricht, me da mucha pena que os vayáis, Múnich no será lo mismo sin vosotros! A mi jerezana: todavía recuerdo esos cafés al salir yo de mis ratoncillos jajaja! A ti también te conocí al final pero aun y así hemos 
vivido mucho juntas, pero todavía tenemos que irnos de vacaciones juntassss (si puede ser a Indonesia a bailar la gozadera jajaja)! Y gracias x la ultima visita a Reus, me hizo mucha ilusión!!!!!

Papi: cuantas risas me he echado contigo madre mía!!! Desde videos, disfraces, carnavales, fiestas, festivales con huracán incluido, wiesn y tacones jajajaja! Tuyo es el record de visitas a Maastricht jajaja! Gracias por todo y nos vemos en Alicante, Sevilla o donde sea!!! Uhhhh que feoooooooooo!

Raquel: a ti te conocí cuando ya no vivías en Múnich jajaja! Pero como buena fiestera siempre en la Oktoberfest! Muchas gracias también por esas vacaciones en Mallorca, descubriendo la isla contigo y Mónica y vuestra visita en carnavales!! Nos vemos pronto en la Oktober en Mallorca o donde haga falta!!!

Gerhard: Vielen Dank für deine große Hilfe - zuerst als mein Deutschtandem und dann mit den ganzen Steuererklärungen! Ich freue mich sehr, dich so glücklich mit deiner Familie zu sehen! Herzlichen Dank für alles!

Y también a vosotros, Daniel, Roman, Candela, Alex, Alberto, Joana, Adolfo, Luis Eduardo, Camilo, Octavio, Anna, David, Marcia y Marcos. Por los buenos momentos y por vuestro interés y apoyo siempre que lo necesito.

Y ahora mi familia!

Primero mis abuelos a los que he dedicado esta tesis. Avis Joan i Josefina, encara que no heu pogut viure aquesta tesis amb mi, us vull donar les gràcies. Especialment a l'àvia, que encara em recordo trucant-la per 
demanar-li ajuda amb rodolins o frases fetes, i perquè sense ella la nit del caga tió ja no es igual. Sempre us portaré amb mi!

$\mathrm{Y}$ a mis yayos Luis y Carmen. Al abuelo que esta como siempre, cada vez más joven y fuerte. Has sido como un segundo padre para mí, todavía recuerdo cuando me venias a recoger al colegio con mi bocadillo!. No me pudiste venir a ver a Múnich pero sí que vendrás a Maastricht y estoy muy contenta que puedas estar conmigo en el día de la defensa de esta tesis!!!

Mi abuela Carmen, hace poco que ya no estas con nosotros, pero se ha hecho muy largo. Gracias por criarme, por educarme y siempre por preocuparte por tu nieta que se iba a Alemania y luego a Holanda. Todavía se me hace muy raro llegar a Reus y no poder verte. Pero sé que estés donde estés siempre me acompañas y me das fuerzas cuando las necesito. Te echo mucho de menos.

A mi hermana Aida, que también siempre ha venido a verme allá donde estuviera. Sabes que siempre estaré ahí cuando me necesites, y yo sé que siempre te tendré también a ti. Esa enfermera!!!!

Victor, Silvia y Luis, también muchas gracias a vosotros, y por visitarme en Múnich. Silvia, mi hermana mayor! Siempre has estado ahí cuando te he necesitado, desde pequeñas en casa de la abuela, y se me ha hecho difícil el no estar juntas desde que me mude. Aun así sé que siempre estaremos unidas y espero más cerca que ahora!

Irina: mi niña. Todavía recuerdo la primera vez que te vi en el hospital, tan pequeñita tan guapa!! Fue muy duro para mí el irme, porque sabía que me iba a perder muchos momentos de tu infancia, pero he intentado siempre estar ahí aunque a veces fuera desde la distancia! No sé 214 
dónde estaré en el futuro, pero sabes que siempre me tendrás ahí cuando lo necesites!!

Araceli y German: gracias! Quizás no hayáis vivido tanto esta tesis pero si vivisteis el master. Gracias por acogerme en Fuenlabrada y por esas cenitas en el bar de abajo! German por todos los documentos que me llegaste a imprimir y las idas y venidas para recoger el titulo!! Me debéis una visita en Múnich y espero que podáis venir a Maastricht! Muchos besos!!!!

Y por supuesto a mis padres! Sé que habéis sufrido viéndome marchar a Múnich, pero siempre me habéis apoyado y habéis entendido que era lo mejor para mí. Para mí tampoco fue fácil irme y no teneros ya conmigo cada día y todavía lo es. Gracias por dejarme seguir mis sueños y entenderlo. Os quiero mucho!!!!

Papa moltes gràcies, per tot! Sé que has treballat molt dur per poguer-me donar una bona educació i per poguer estudiar a Barcelona. També per ajudar-me al principi a Múnich que no va ser fàcil! He après molt de tu, i encara continuo fent-ho.

Mama muchísimas gracias! Tú también has trabajado muy duro, para que yo pueda estar hoy donde estoy. Sé que te cuesta el no tenerme cerca, pero sabes que aunque sea en la distancia siempre voy a estar ahí. Gracias también por esas maletas de comida que tanto yo como mis amigos hemos disfrutado! Te echo mucho de menos, pero tú sabes que estoy bien y que poco podría haber hecho si me hubiera quedado en Reus. No sé dónde me llevara el futuro pero siempre estaré ahí! 
Dank je wel Diane, Eddy, Nicole, Wim, Astrid en Tim om mij te behandelen als een lid van de familie en voor jullie interesse in dit proefschrift.

And now my guapo!!! I don't know actually where to begin.... Many thanks first as my friend, for all my questions and difficulties with bureaucracy, "belasting", statistics, whatever it was you were always there. You were also always in the Alla jajajaja! For the many trips to Munich, Berlin, Ibiza, Valencia, Paris, Andalucía, Girona and the many more that will come. Now as my partner, for your advice, your support and all your help, especially in the last months. You make my life much happier and you always know how to make me smile. I always told you that I didn't expect to find you here, but I will be always grateful for that. Now your turn will come to make really official what your Belgium shirt says jajaja! and you will have me always there. I don't know what the future will bring us or where we will go, but it doesn't matter as long as you are with me. Looking forward to the next chapter together!!!! 


\section{Curriculum vitae}


Yara Pujol López was born on the 25th of March 1985 in Reus, Spain. She obtained her High School degree with specialization in Health Sciences at the Col·legi Sant Josep (Reus, Spain) in 2003. Next, she moved to Barcelona and obtained her bachelor's degree in Biology in 2008 with specialization in Sanitary Biology at the Universitat Autònoma de Barcelona (Barcelona, Spain). In her hometown, she conducted her final internship in the Center of Human Reproduction Biogest, (Reus, Spain) under the supervision of Carolina Matas. Immediately afterwards, she started her Master in Human Reproduction in the Universidad Complutense de Madrid (Madrid, Spain), conducting her final internship in the Center of Infertility and Human Reproduction ( $\mathrm{CIRH}$, Barcelona, Spain). Her next step took her away from the academic world and moved to San Francisco, USA to improve her English. Yara then rejoined the academic world and moved to Munich, Germany. In 2011 she began her PhD at the Clinic of Psychiatry and Psychotherapy at the Ludwig-Maximilians-University (LMU) under the supervision of Dr. Aye-Mu Myint. In 2013, she moved to Maastricht, The Netherlands to continue her PhD at the School of Mental Health and Neurosciences, Maastricht University under the supervision of Prof. Harry Steinbusch, Dr. Daniel van den Hove and Dr. Gunter Kenis. In her PhD project she investigated the developmental and psychoneuroimmunological mechanisms in depression. The results of this work are described on this thesis. 
Publication list 


\section{Peer-reviewed publications}

* Pujol Lopez Y, Steinbusch HWM, Rutten BPF, Kenis G, van den Hove D, Myint AM.

Effects of subcutaneous lipopolysaccharide (LPS) injection on gestational length and intrauterine and neonatal mortality in mice. Neuroimmunomodulation 2015;22(4):274-8

\section{Submitted / under review manuscripts}

* Pujol Lopez Y, Kenis G, Stettinger W, Neumeier K, de Jonge S, Steinbusch HWM, Zill P, van den Hove D, Myint AM.

Effects of prenatal polyinosinic:polycytidylic acid (Poly I:C) exposure on global histone deacetylase (HDAC) and DNA methyltransferase (DNMT) activity in the mouse brain.

* Pujol Lopez Y, Kenis G, Rutten BPF, Myint AM, Steinbusch HWM ,van den Hove D.

Quinolinic acid-immunoreactivity in the naïve mouse brain.

\section{Manuscripts in preparation}

* Pujol Lopez Y, van den Hove D, Steinbusch HWM, Kenis ${ }^{1}{ }^{1}$, Myint AM ${ }^{1}$.

Prenatal maternal infection and offspring brain development: programming of adult psychopathology (Review).

${ }^{1}$ Authors contributed equally to this work

* Pujol Lopez Y, Leue C, Koek G, Myint AM, van den Hove D, Pishva E, Schwarz MJ, van Os J, Steinbusch HWM, Rutten BPF, Kenis G.

Depressive symptoms during IFN-alpha therapy in humans associated with changes in the kynurenine pathway. 
Neuroscience

\section{3}

Rob Havermans: Bipolar disorder in daily life; Mood and cortisol responses to naturally occurring events. Supervisor: Prof.dr. M. de Vries; Co-Supervisor: Dr. N. Nicolson.

Véronique Moers-Hornikx: Deep brain stimulation and the cerebellum. Supervisors:

Prof.dr. J. Vles / Prof.dr. Y. Temel; Co-Supervisor: Dr. G. Hoogland.

Nicole Veldhorst-Janssen: Intranasal delivery of rapid acting drugs. Supervisors: Prof.dr. M. Marcus / Prof.dr. C. Neef; Co-Supervisor: Dr. P.H. van der Kuy.

Stéphanie Knippenberg: Vitamin D and Multiple Sclerosis: immunological and clinical outcome. Supervisor: Prof.dr. J. Cohen-Tervaert; Co-Supervisors: Dr. J. Damoiseaux / Dr. Y. Bols.

Erik D. Gommer: Dynamic Cerebral Autoregulation: from methodology towards clinical application. Supervisors: Prof.dr. W.H. Mess / Prof.dr. R.B. Panerai, UK; Co-Supervisor: Dr.ir. J.P.H. Reulen.

Olga A.H. Reneerkens: Can PDE inhibition improve cognition? Translational insights. Supervisor: Prof.dr. H.W.M. Steinbusch; Co-Supervisor: Dr. J. Prickaerts.

Lyzel S. Elias-Sonnenschein: Clinical and biomarker correlates of genetic risk factors for Alzheimer's disease. Supervisor: Prof.dr. F.R.J. Verhey; Co-Supervisor: Dr. P.J. Visser.

Diego F. Mastroeni: Epigenetic Dysregulation and the Pathophysiology of Alzheimer's

Disease. Supervisors: Prof.dr. H.W.M. Steinbusch / Prof.dr. P.D. Coleman, Sun City, Arizona; CoSupervisors: Dr. B.P.F. Rutten / Dr. D.L.A. van den Hove.

Leonidas Chouliaras: Epigenetic Regulation in Aging and Alzheimer's disease: A

translational perspective. Supervisor: Prof.dr. H.W.M. Steinbusch; Co-Supervisors: Dr. B.P.F. Rutten / Dr. D.L.A. van den Hove.

Liesbeth Knaepen: Perinatal events and altered pain sensitivity in later life. Supervisors: Prof.dr. E.A.J. Joosten / Prof.dr. D. Tibboel, EUR; Co-Supervisor: Dr. J. Patijn.

Marisela Martinez-Claros: Hippocampal plasticity and corticosterone: From dendrites to behaviour. Supervisor: Prof.dr. H.W.M. Steinbusch; Co-Supervisors: Dr. J.L. Pawluski /Dr. J.

Prickaerts.

Marcus D. Lancé: A circle of improvement in bleeding management: from laboratory to clinic and back. Supervisors: Prof.dr. M.A.E. Marcu / Prof.dr. J.W.M. Heemskerk; Co-Supervisor: Dr. Y.M.C. Henskens.

Hilde Braakman: Imaging the brain; neuronal correlates of cognitive impairment in children with frontal lobe epilepsy. Supervisors: Prof.dr. A.P. Aldenkamp / Prof.dr. J.S.H. Vles; CoSupervisors: Dr.ir. W.H. Backes / Dr. P.A.M. Hofman.

Willem $\mathrm{H}$. van Zwam: Aneurysmal subarachnoid hemorrhage: imaging strategies and cost-effectiveness aspects in diagnostic work-up and post-therapeutic follow-up. Supervisors: Prof.dr. J.T. Wilmink / Prof.dr. J.E. Wildberger; Co-Supervisor: Dr. P.A.M. Hofman.

Klara De Cort: The Pathogenesis of Panic Disorder. Supervisors: Prof.dr. I. Myin-Germeys / Prof.dr. E.J.L. Griez; Co-Supervisors: Dr. K.R.J. Schruers / Dr. I. Van Diest, Leuven.

Kim van Wijck: Mind the Gap; experimental studies on splanchnic hyperfusion and gastrointestinal integrity loss in man. Supervisors: Prof.dr. W.A. Buurman / Prof.dr. C.H.C. Dejong; 
Co-Supervisor: Dr. K. Lenaerts.

Yvette Roke: Antipsychotic-induced hyperprolactinemia in children and adolescents with mainly autism spectrum disorders. Prevalence, symptoms, clinical consequences and genetic risk factors. Supervisors: Prof.dr. P.N. van Harten / Prof.dr. J.K. Buitelaar (RUN); Co-Supervisor: Dr. A. Boot (UMCG).

Fleur Goezinne: Retinal detachment surgery: pre and postoperative prognostic factors. Supervisors: Prof.dr. F. Hendrikse / Prof.dr. C.A.B. Webers; Co-Supervisor: Dr. E.C. La Heij (Amsterdam).

Ralph L.J.G. Maassen: The Merits of Videolaryngoscopy during Glottic Visualisation for Endotracheal Intubation. Supervisors: Prof.dr. M. Marcus / Prof.dr. A. van Zundert (University of Queensland).

Maria J. de Sousa Guerreiro: The role of sensory modality in age-related distraction. Supervisor: Prof.dr. C.M. van Heugten; Co-Supervisor: Dr. P.W.M. van Gerven.

Ine Rayen: Effects of developmental fluoxetine exposure on neurobehavioral outcomes. Supervisor: Prof.dr. H.W.M. Steinbusch; Co-Supervisors: Dr. J.L. Pawluski / Dr. T.D. Charlier (Ohio University, USA).

Nynke M.G. Bodde: Psychogenic non-epileptic seizures; a separate disorder or part of a continuum? Supervisors: Prof.dr. R. van Oostenbrugge / Prof.dr. K. Vonck (UZ Gent); Co-Supervisors: Dr. R. Lazeron / Dr. A. de Louw (Epilepsiecentrum Kempenhaeghe, Heeze).

Alejandro M. Gomez: Novel strategies for making myasthenia less gravis: targeting plasma cells and the neuromuscular junction. Supervisor: Prof.dr. M.H. De Baets; Co-Supervisors: Dr. M. Losen / Dr. P. Martinez-Martinez.

Mohammad S. Rahnama'i: Prostaglandins and Phosphodiesterases in the Urinary Bladder Wall. Supervisors: Prof.dr. Ph. Van Kerrebroeck / Prof.dr. S. de Wachter (Universiteit Antwerpen); Co-Supervisor: Dr. G. van Koeveringe.

Mariken B. de Koning: Studying biomarkers in populations at genetic and clinical high risk for psychosis. Supervisors: Prof.dr. T. Amelsvoort / Prof.dr. J. Booij (AMC).

Fabien Boulle: Epigenetic regulation of BDNF/TrkB signaling in the pathophysiology and treatment of mood disorders. Supervisors: Prof.dr. H.W.M. Steinbusch / Prof.dr. L. Lanfumey (Universiteit Parijs); Co-Supervisors: Dr. D. van den Hove / Dr. G. Kenis.

\section{4}

Iris Nowak-Maes: Tinnitus; assessment of quality of life $\&$ cost-effectiveness.

Supervisors: Prof.dr. M. Peters / Prof.dr. B. Kremer; Co-Supervisors: Dr. M. Joore / Dr. L. Anteunis.

Marjolein Huijts: Cognitive function in patients with cerebral small vessel disease.

Supervisor: Prof.dr. R.J. van Oostenbrugge; Co-Supervisors: Dr. A.A. Duits / Dr. J. Staals.

Markus Gantert: Fetal inflammatory injury as origin of long term disease: Lessons from animal models. Supervisors: Prof.dr. B. Kramer / Prof.dr. L. Zimmermann; Co-Supervisor: Dr. A. Gavilanes.

Elke Kuypers: Fetal development after antenatal exposures: Chorioamnionitis and maternal glucocorticoids. Supervisors: Prof.dr. B.W. Kramer / Prof.dr. H.W. Steinbusch / Prof.dr. Suhas G. Kallapur (University of Cincinnati, Ohio, USA).

Pieter Kubben: Ultra low-field strength intraoperative MRI for Glioblastoma Surgery. Supervisor: Prof.dr. J.J. van Overbeeke; Co-Supervisor: Dr. H. van Santbrink.

Laura Baijens: Surface electrical stimulation of the neck for oropharyngeal dysphagia in Parkinson's disease: therapeutic aspects and reliability of measurement. Supervisor: Prof.dr. B. Kremer; Co-Supervisor: Dr. R. Speyer, Townsville.

Janneke Hoeijmakers: Small fiber neuropathy and sodium channels; a paradigm shift. Supervisor: Prof.dr. R.J. van Oostenbrugge; Co-Supervisors: Dr. C.G. Faber / Dr. I.S.J. Merkies. 
Stephanie Vos: The Role of biomarkers in preclinical and prodromal Alzheimer's disease. Supervisor: Prof.dr. F.R. Verhey; Co-Supervisor: Dr. P.J. Visser.

Muriël Doors: The Value of Optical Coherence Tomography in Anterior Segment Surgery. Supervisors: Prof.dr. R.M. Nuijts / Prof.dr. C.A. Webers; Co-Supervisor: Dr. T.T.J.M. Berendschot.

Anneke Maas: Sleep problems in individuals with genetic disorders associated with intellectual disability. Supervisors: Prof.dr. I. Curfs / Prof.dr. R. Didden.

Sebastiaan van Gorp: Translational research on spinal cord injury and cell-based therapies; a focus on pain and sensorimotor disturbances. Supervisors: Prof.dr. B. Joosten / Prof.dr. M. van Kleef; Co-Supervisors: Dr. J. Patijn /Dr. R. Deumens, KU Leuven.

Andrea Sannia: High risk newborns and brain biochemical monitoring. Supervisor: Prof.dr. J.S.H. Vles; Co-Supervisors: Dr. D. Gazzolo, Alessandria, Italy / Dr. A.W.D. Gavilanes.

Julie A.D.A. Dela Cruz: Dopamine mechanisms in learning and memory: Evidence from rodent studies. Supervisors: Prof.dr. H.W.M. Steinbusch / Prof.dr. R.J. Bodnar, New York; CoSupervisor: Dr. B.P.F. Rutten.

René Besseling: Brain wiring and neuronal dynamics; advances in MR imaging of focal epilepsy. Supervisors: Prof.dr. A.P. Aldenkamp / Prof.dr.ir. W.H. Backes; Co-Supervisor: Dr. J.F.A. Jansen.

Maria Quint-Fens: Long-term care after stroke; development and evaluation of a longterm intervention in primary care. Supervisors: Prof.dr. J.F.M. Metsemakers / Prof.dr. C.M. van Heugten / Prof.dr. M. Limburg, Almere; Co-Supervisor: Dr. G.H.M.I. Beusmans.

Veronique Moulaert: Life after survival of a cardiac arrest; the heart of the matter.

Supervisors: Prof.dr. J.A. Verbunt / Prof.dr. C.M. van Heugten / Prof.dr. D.T. Wade, Oxford, UK. Feikje Smeets: The hallucinatory-delusional state: a crucial connection in the psychosis symptom network. Supervisor: Prof.dr. J. van Os; Co-Supervisor: Dr. T. Lataster.

Lies Clerx: Alzheimer's disease through the MR-eye; novel diagnostic markers and the road to clinical implementation". Supervisor: Prof.dr. F. Verhey; Co-Supervisors:

Dr. P.J. Visser / P. Aalten.

Sonny Tan: The subthalamic nucleus in Parkinson's disease. Supervisors: Prof.dr. Y. Temel / Prof.dr. H.W.M. Steinbusch / Prof.dr. T. Sharp, Oxford, UK / Prof.dr. V. Visser-Vandewalle, Koln. Koen van Boxem: The use of pulsed radiofrequency in the management?of chronic lumbosacral radicular pain. Supervisors: Prof.dr. M. van Kleef / Prof.dr. E.A.J. Joosten; CoSupervisor: Assoc. Prof.dr. J. van Zundert.

Jérôme Waterval: Hyperostosis cranialis interna. Supervisors: Prof.dr. J.J. Manni / Prof.dr. R.J. Stokroos.

Sylvie Kolfschoten-van der Kruijs: Psychogenic non-epileptic seizures; the identification of neurophysiological correlates. Supervisors: Prof.dr. A.P. Aldenkamp / Prof.dr. K.E.J. Vonck, Universiteit Gent; Co-Supervisors: Dr. J.F.A. Jansen / Dr. R.H.C. Lazeron, Kempenhaeghe.

Wouter Pluijms: Spinal cord stimulation and pain relief in painful diabetic:

polyneuropathy, a translational approach. Supervisors: Prof.dr. M. van Kleef / Prof.dr. E.A. Joosten; Co-supervisor: Dr. C.G. Faber.

Ron Handels: Health technology assessment of diagnostic strategies for Alzheimer's disease. Supervisors: Prof.dr. F.R.J. Verhey / Prof.dr. J.L. Severens (EUR); Co-Supervisor: Dr. M.A. Joore / Dr. C.A.G. Wolfs.

Evelyn Peelen: Regulatory T cells in the pathogenesis of Multiple Sclerosis: potential targets for vitamin D therapy. Supervisors: Prof.dr. R.M.M. Hupperts / Prof.dr. J.W. Cohen Tervaert; Co-Supervisor: Dr. J.G.M.C. Damoiseaux / Dr. M.M.G.L.Thewissen, Diepenbeek.

Reint Jellema: Cell-based therapy for hypoxic-ischemic injury in the preterm brain. Supervisors: Prof.dr. B.W.W. Kramer / Prof.dr. H.W.M. Steinbusch; Co-Supervisor: Dr. W.T.V. Germeraad / Dr. P. Andriessen, Veldhoven.

Maria Wertli: Prognosis of Chronic Clinical Pain Conditions: The Example of Complex Regional Pain Syndrome 1 and Low Back Pain. Supervisors: Prof.dr. M. van Kleef; Co-Supervisor: Dr. 
F. Brunner, Zürich / Dr. R. Perez, VUmc.

Dagmar Zeef: An experimental model of Huntington's disease: Validation \& Stimulation.

Supervisors: Prof.dr. Y. Temel / Prof.dr. H.W.M. Steinbusch; Co-supervisor: Dr. A. Jahanshahi.

Jeroen Decoster: Breaking Down Schizophrenia into phenes, genes and environment.

Supervisors: Prof.dr. I. Myin-Germeys / Prof.dr. M. De Hert, KU Leuven; Co-Supervisor: Dr. R. van Winkel.

Eaja Anindya Sekhar Mukherjee: Fetal Alcohol Spectrum Disorders: exploring prevention and management. Supervisor: Prof.dr. L.M.G. Curfs; Co-Supervisor: Prof. S. Hollins, St. George's University of London, UK.

Catherine van Zelst: Inside out; On stereotype awareness, childhood trauma and stigma

in psychosis. Supervisors: Prof.dr. Ph. Delespaul / Prof.dr. J. van Os.

Ibrahim Tolga Binbay: Extended Psychosis Phenotype in the Wider Social Environment.

Supervisor: Prof.dr. J. van Os; Co-Supervisor: Dr. M. Drukker.

Frank Van Dael: OCD matters in psychosis. Supervisors: Prof.dr. J. van Os / Prof.dr. I. Myin-

Germeys.

Pamela Kleikers: NOXious oxidative stress: from head toe too and back. Supervisors:

Prof.dr. H.H.H.W. Schmidt / Prof.dr. H.W.M. Steinbusch; Co-Supervisor: Dr. B. Janssen.

José Luis Gerardo Nava: In vitro assay systems in the development of therapeutic

interventions strategies for neuroprotection and repair. Supervisors: Prof.dr.med. J. Weis / Prof.dr.

H.W.M. Steinbusch; Co-Supervisor: Dr. G.A. Brook, RWTH Aachen.

Eva Bollen: Cyclic nucleotide signaling and plasticity. Supervisors: Prof.dr. H.W.M.

Steinbusch / Prof.dr. R. D'Hooge, KU Leuven; Co-Supervisor: Dr. J. Prickaerts.

\section{5}

Jessica A. Hartmann: A good laugh and a long sleep; Insights from prospective and ambulatory assessments about the importance of positive affect and sleep in mental health. Supervisor: Prof.dr. J. van Os; Co-Supervisors: C.J.P. Simons / Dr. M. Wichers.

Bart Ament: Frailty in old age; conceptualization and care innovations. Supervisors:

Prof.dr. G.I.J.M. Kempen / Prof.dr. F.R.J. Verhey; Co-Supervisor: Dr. M.E. de Vugt.

Mayke Janssens: Exploring course and outcome across the psychosis-continuum.

Supervisor: Prof.dr. I. Myin-Germeys; Co-Supervisor: Dr. T. Lataster.

Dennis M.J. Hernau: Dopayours is not dopamine: genetic, environmental and

pathological variations in dopaminergic stress processing. Supervisor: Prof.dr. I. Myin-Germeys; CoSupervisors: Prof.dr. F.M. Mottaghy / Dr. D. Collip.

Ingrid M.H. Brands: The adaptation process after acquired brain injury Pieces of the puzzle. Supervisors: Prof.dr. C.M. van Heugten / Prof.dr. D.T. Wade, Oxford UK; Co-Supervisors: Dr. S.Z. Stapert / Dr. S. Köhler.

Francesco Risso: Urinary and salivary S100B monitoring in high risk infants. Supervisor:

Prof.dr. J.S.H. Vles; Co-Supervisors: Dr. D. Gazzolo, Genoa, Italy / Dr. A.W.D. Gavilanes.

Alessandro Borghesi: Stem and Progenitor Cells in Preterm Infants: Role in the

Pathogenesis and Potential for Therapy. Supervisor: Prof.dr. L. Zimmermann; Prof.dr. B. Kramer;

Co-Supervisors: Dr. D. Gazzolo, Genoa,Italy / Dr. A.W.D. Gavilanes.

Claudia Menne-Lothmann: Affect dynamics; A focus on genes, stress, and an opportunity

for change. Supervisor: Prof.dr. J. van Os; Co-Supervisors: Dr. M. Wichers / Dr. N. Jacobs.

Martine van Nierop: Surviving childhood new perspectives on the link between childhood

trauma and psychosis. Supervisors: Prof.dr. I. Myin-Germeys / Prof.dr. J. van Os; Co-Supervisor: Dr.

R. van Winkel.

Sylvia Klinkenberg: VNS in children; more than just seizure reduction. Supervisors: Prof.dr.

J. Vles / Prof.dr. A. Aldenkamp; Co-Supervisor: Dr. H. Majoie. 
Anouk Linssen: Considerations in designing an adult hearing screening programme. Supervisor: Prof.dr. B. Kremer; Co-Supervisors: Dr. L. Anteunis / Dr. M. Joore.

Janny Hof: Hearing loss in young children; challenges in assessment and intervention. Supervisors: Prof.dr. B. Kremer / Prof.dr. R. Stokroos / Prof.dr. P. van Dijk, RUG; Co-Supervisor: Dr. L. Antheunis.

Kimberly Cox-Limpens: Mechanisms of endogenous brain protection; Clues from the transcriptome. Supervisors: Prof.dr. J. Vles / Prof.dr. L. Zimmermann; Co-Supervisor: Dr. A. Gavilanes.

Els Vanhoutte: Peripheral Neuropathy outcome measures; Standardisation (PeriNomS) study part 2: Getting consensus. Supervisors: Prof.dr. C. Faber / Prof.dr. P. van Doorn; CoSupervisor: Dr. I. Merkies, Spaarne ziekenhuis Hoofddorp.

Mayienne Bakkers: Small fibers, big troubles; diagnosis and implications of small fiber neuropathy. Supervisors: Prof.dr. C. Faber / Prof.dr. M. de Baets; Co-Supervisor: Dr. I. Merkies, Spaarne ziekenhuis Hoofddorp.

Ingrid Kramer: Zooming into the micro-level of experience: An approach for understanding and treating psychopathology. Supervisor: Prof.dr. J. van Os; Co-Supervisors: Dr. M. Wichers, UMC Groningen / Dr. C. Simons.

Esther Bouman: Risks and Benefits of Regional Anesthesia in the Perioperative Setting. Supervisors: Prof.dr. M. van Kleef / Prof.dr. M. Marcus, HMC, Qatar / Prof.dr. E. Joosten; CoSupervisor: Dr. H. Gramke.

Mark Janssen: Selective stimulation of the subthalamic nucleus in Parkinson's disease; dream or near future. Supervisors: Prof.dr. Y. Temel / Prof.dr. V. Visser-Vandewalle, Keulen / Prof.dr. A. Benazzouz, Bordeax, France.

Reina de Kinderen: Health Technology Assessment in Epilepsy; economic evaluations and preference studies. Supervisors: Prof.dr. S. Evers / Prof.dr. A. Aldenkamp; Co-Supervisor: Dr. H. Majoie / Dr. D. Postulart, GGZ O-Brabant.

Saskia Ebus: Interictal epileptiform activity as a marker for clinical outcome. Supervisors: Prof.dr. A. Aldenkamp / Prof.dr. J. Arends, TUE / Prof.dr. P. Boon, Universiteit Gent, België.

Inge Knuts: Experimental and clinical studies into determinants of panic severity. Supervisor: Prof.dr. I. Myin-Germeys; Co-Supervisor: Dr. K. Schruers; Influencing panic.

Nienke Tielemans: Proactive coping post stroke: The Restored4Stroke Self-Management study. Supervisors: Prof.dr. C. van Heugten / Prof.dr. J. Visser-Meily, UMC Utrecht; Co-Supervisor: Dr. V. Schepers, UMC Utrecht.

Tom van Zundert: Improvements Towards Safer Extraglottic Airway Devices. Supervisors: Prof.dr. A.E.M. Marcus / Prof.dr. W. Buhre / Prof.dr. J.R. Brimacombe, Queensland, Australia / Prof.dr. C.A. Hagberg.

Tijmen van Assen: Anterior Cutaneous Nerve Entrapment Syndrome Epidemiology and surgical management. Supervisors: Prof.dr. G.L. Beets / Prof.dr. M. van Kleef / Dr. R.M.H. Roumen / Dr. M.R.M. Scheltinga, MMC Veldhoven.

Rohit Shetty: Understanding the Clinical, Immunological and Genetic Molecular Mechanisms of Keratoconus. Supervisors: Prof.dr. R.M.M.A. Nuijts / Prof.dr. C.A.B. Webers.

Christine van der Leeuw: Blood, bones and brains; peripheral biological endophenotypes and their structural cerebral correlates in psychotic disorder. Supervisor: Prof.dr. J. van Os; Cosupervisor: Dr. M. Marcelis.

Sanne Peeters: The Idle Mind Never Rests; functional brain connectivity across the psychosis continuum. Supervisor: Prof.dr. J. van Os; Co-supervisor: Dr. M. Marcelis.

Nick van Goethem: $\boldsymbol{\alpha} 7$ nicotinic acetylcholine receptors and memory processes: mechanistic and behavioral studies. Supervisor: Prof.dr. H.W.M. Steinbusch; Co-supervisor: Dr. J. Prickaerts.

Nicole Leibold: A Breath of fear; a translational approach into the mechanisms of panic. Supervisor: Prof.dr. H.W.M. Steinbusch; Co-supervisors: Dr. K.R.J. Schruers / Dr. D.L.A. van den Hove. 
Renske Hamel: The course of mild cognitive impairment and the role of comorbidity. Supervisor: Prof.dr. F.R.J. Verhey; Co-supervisors: Dr. I.H.G.B. Ramakers / Dr. P.J. Visser. 
The future belongs to those who believe in the beauty of their dreams.

(Eleanor Roosevelt) 
